Prepared in cooperation with the Wyoming Department of Agriculture on behalf of the Wyoming Ground-water and Pesticides Strategy Committee

\title{
Pesticides in Wyoming Groundwater, 2008-10
}

Scientific Investigations Report 2013-5064 
Cover. Bighorn Basin geographic area. Photograph by Cheryl Eddy-Miller, U.S. Geological Survey. 


\section{Pesticides in Wyoming Groundwater, 2008-10}

By Cheryl A. Eddy-Miller, Timothy T. Bartos, and Michelle L. Taylor

Prepared in cooperation with the Wyoming Department of Agriculture on behalf of the Wyoming Ground-water and Pesticides Strategy Committee

Scientific Investigations Report 2013-5064 


\section{U.S. Department of the Interior \\ KEN SALAZAR, Secretary}

\section{U.S. Geological Survey \\ Suzette M. Kimball, Acting Director}

U.S. Geological Survey, Reston, Virginia: 2013

For more information on the USGS - the Federal source for science about the Earth, its natural and living resources, natural hazards, and the environment, visit http://www.usgs.gov or call 1-888-ASK-USGS.

For an overview of USGS information products, including maps, imagery, and publications, visit http://www.usgs.gov/pubprod

To order this and other USGS information products, visit http://store.usgs.gov

Any use of trade, firm, or product names is for descriptive purposes only and does not imply endorsement by the U.S. Government.

Although this information product, for the most part, is in the public domain, it also may contain copyrighted materials as noted in the text. Permission to reproduce copyrighted items must be secured from the copyright owner.

Suggested citation:

Eddy-Miller, C.A., Bartos, T.T., and Taylor, M.L., 2013, Pesticides in Wyoming Groundwater, 2008-10: U.S. Geological Survey Scientific Investigations Report 2013-5064, 45 p. 


\section{Acknowledgments}

The authors gratefully acknowledge the generous assistance of landowners throughout Wyoming who provided access to their property and wells for the sampling. The authors also acknowledge assistance with site selection from the many Conservation Districts and County Weed and Pest Districts in Wyoming. 


\section{Contents}

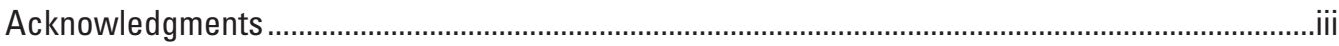

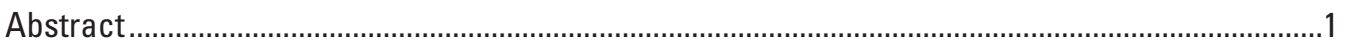

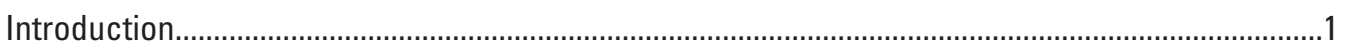

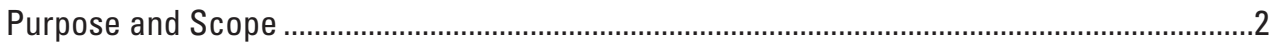

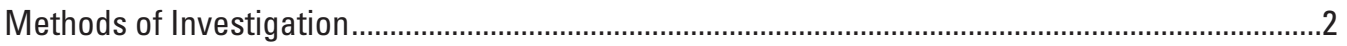

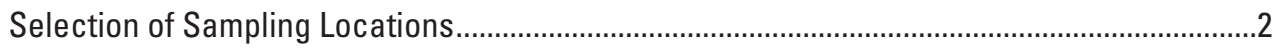

Sample Collection and Chemical Analyses ......................................................................

Data Reporting and Treatment..........................................................................................

Laboratory Reporting Levels ......................................................................................

Recensoring and Assessment Levels ............................................................................

Quality-Control Samples and Quality Assurance ................................................................14

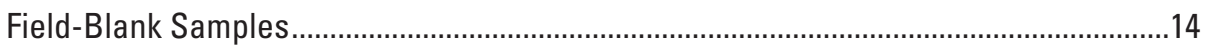

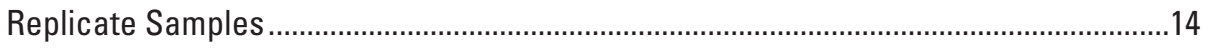

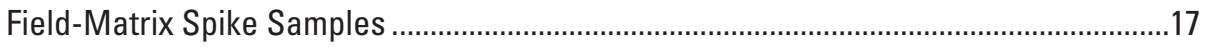

Pesticide 0ccurrence during 2008-10 Resampling Study ..............................................................17

Pesticides Detected during 2008-10 Resampling Study .....................................................17

Pesticide Detection Frequency by Well During the 2008-10 Resampling Study.....................19

Pesticide Detections by Action During the 2008-10 Resampling Study.................................19

Differences in Pesticide Occurrence between 1995-2006 Baseline and 2008-10 Resampling

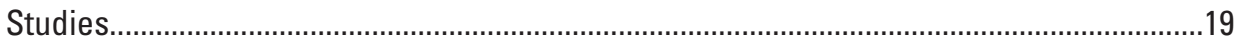

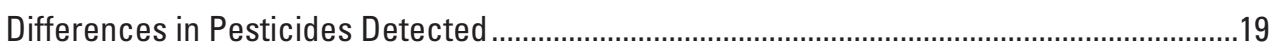

Differences in Pesticide Detections ....................................................................................

Differences in Pesticide Concentrations ................................................................................19

Differences in Pesticide Detections and Detection Frequency Relative to Land Use and

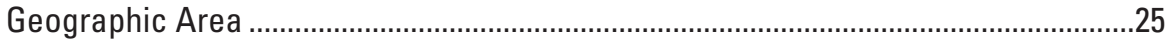

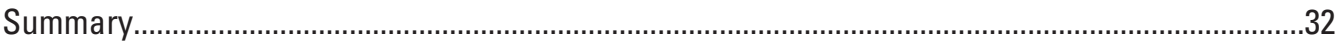

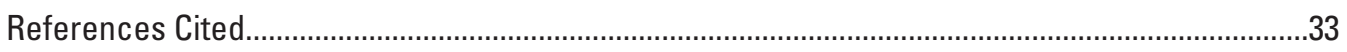

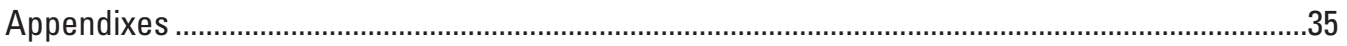

\section{Figures}

1. Map showing wells sampled from the eight geographic areas of Wyoming during the 1995-2006 baseline study.

2. Map showing location of, and land use near, the 52 wells sampled during the 2008-10 resampling study, Wyoming...

3. Graph showing pesticide detections and concentrations (using no assessment level) in groundwater samples collected from 52 wells during the 2008-10 resampling study, Wyoming

4. Graph showing number of pesticides detected in groundwater samples collected from 52 wells sampled during the 2008-10 resampling study (using no assessment level), compound-specific assessment level, and common assessment level, Wyoming. 
5. Graph showing pesticide detections relative to pesticide action in groundwater samples collected from 52 wells sampled during the 2008-10 resampling study, Wyoming.

6. Graph showing pesticide detections and concentrations (using no assessment level) in groundwater samples collected from 52 wells during the 1995-2006 baseline study

7. Pie diagram showing difference in number of pesticides detected in the same well between the 1995-2006 baseline and 2008-10 resampling studies

8. Graphs showing pesticide detections and concentrations in groundwater samples collected from 52 wells during 1995-2006 baseline study and 2008-10 resampling study, Wyoming.....

9. Graphs showing percentage of wells with at least one pesticide detected in groundwater samples collected from 52 wells relative to land use and geographic area during the 1995-2006 baseline study and the 2008-10 resampling study, Wyoming.

10. Pie diagrams showing difference in number of pesticides detected in the same well between 1995-2006 baseline and 2008-10 resampling studies.

\section{Tables}

1. Pesticides analyzed in 1995-2006 baseline and 2008-10 resampling studies, with trade names, pesticide actions, pesticide classes, laboratory reporting levels, and U.S. Environmental Protection Agency drinking-water standards

2. Pesticides detected in groundwater samples collected from 52 wells sampled during both 1995-2006 baseline study and 2008-10 resampling study...

3. Summary of pesticide detections and concentrations in groundwater sampled during the 2008-10 resampling study, Wyoming

4. Pesticides detected in the 52 wells sampled during 1995-2006 baseline study and (or) 2008-10 resampling study, Wyoming ...

5. Differences in pesticide detections between the 1995-2006 baseline study and the 2008-10 resampling study, Wyoming.

Appendix 1. Blank data for sample collected at site 410817104470201, November 2009 ..............36

Appendix 2. Replicate sample data for detected pesticides from samples collected at sites 4108108104223501 (September 2009) and 444603108383401 (September 2010).

Appendix 3. Spike recovery data from sample collected from site 41101910405130 (Lr7), September 2008.

Appendix 4. Name, number, and ancillary information for 52 wells sampled during 1995-2006 baseline study and 2008-10 resampling study, Wyoming

Appendix 5. Summary of pesticides distributed within Wyoming, 2007 


\section{Conversion Factors}

\begin{tabular}{|c|c|c|}
\hline Multiply & By & To obtain \\
\hline \multicolumn{3}{|c|}{ Length } \\
\hline inch (in.) & 2.54 & centimeter $(\mathrm{cm})$ \\
\hline inch (in.) & 25.4 & millimeter $(\mathrm{mm})$ \\
\hline foot $(\mathrm{ft})$ & 0.3048 & meter (m) \\
\hline mile (mi) & 1.609 & kilometer (km) \\
\hline
\end{tabular}

Temperature in degrees Celsius $\left({ }^{\circ} \mathrm{C}\right)$ may be converted to degrees Fahrenheit ( $\left.{ }^{\circ} \mathrm{F}\right)$ as follows:

${ }^{\circ} \mathrm{F}=\left(1.8 \times^{\circ} \mathrm{C}\right)+32$

Horizontal coordinate information is referenced to the North American Datum of 1983 (NAD 83).

Concentrations of chemical constituents in water are given in micrograms per liter $(\mu \mathrm{g} / \mathrm{L})$.

\section{Abbreviations}

CAL common assessment level

CSAL compound-specific assessment level

E estimated

GPSC Ground-water and Pesticides Strategy Committee

KSOCRL U.S. Geological Survey Organic Chemistry Research Laboratory in Lawrence, Kansas

LHA Lifetime Health Advisory

LRL laboratory reporting level

LT-MDL long-term method detection level

MCL Maximum Contaminant Level

MDL method detection limit

MRL minimum reporting level

NAL no assessment level

NLCD National Land Cover Data

NWQL National Water Quality Laboratory of U.S. Geological Survey

QA quality assurance

OC quality control

RPD relative percentage difference

SMP State of Wyoming Generic Management Plan for Pesticides in Ground Water

USEPA U.S. Environmental Protection Agency

USGS U.S. Geological Survey

WDA Wyoming Department of Agriculture

WDEO Wyoming Department of Environmental Quality 


\title{
Pesticides in Wyoming Groundwater, 2008-10
}

\author{
By Cheryl A. Eddy-Miller, Timothy T. Bartos, and Michelle L. Taylor
}

\section{Abstract}

Groundwater samples were collected from 296 wells during 1995-2006 as part of a baseline study of pesticides in Wyoming groundwater. In 2009, a previous report summarized the results of the baseline sampling and the statistical evaluation of the occurrence of pesticides in relation to selected natural and anthropogenic (human-related) characteristics.

During 2008-10, the U.S. Geological Survey, in cooperation with the Wyoming Department of Agriculture, resampled a subset (52) of the 296 wells sampled during 1995-2006 baseline study in order to compare detected compounds and respective concentrations between the two sampling periods and to evaluate the detections of new compounds. The 52 wells were distributed similarly to sites used in the 1995-2006 baseline study with respect to geographic area and land use within the geographic area of interest.

Because of the use of different types of reporting levels and variability in reporting-level values during both the 1995-2006 baseline study and the 2008-10 resampling study, analytical results received from the laboratory were recensored. Two levels of recensoring were used to compare pesticides - a compound-specific assessment level (CSAL) that differed by compound and a common assessment level (CAL) of 0.07 microgram per liter. The recensoring techniques and values used for both studies, with the exception of the pesticide 2,4-D methyl ester, were the same. Twenty-eight different pesticides were detected in samples from the 52 wells during the 2008-10 resampling study. Pesticide concentrations were compared with several U.S. Environmental Protection Agency drinking-water standards or health advisories for finished (treated) water established under the Safe Drinking Water Act. All detected pesticides were measured at concentrations smaller than U.S. Environmental Protection Agency drinking-water standards or health advisories where applicable (many pesticides did not have standards or advisories).

One or more pesticides were detected at concentrations greater than the CAL in water from 16 of 52 wells sampled (about 31 percent) during the resampling study. Detected pesticides were classified into one of six types: herbicides, herbicide degradates, insecticides, insecticide degradates, fungicides, or fungicide degradates. At least 95 percent of detected pesticides were classified as herbicides or herbicide degradates.
The number of different pesticides detected in samples from the 52 wells was similar between the 1995-2006 baseline study (30 different pesticides) and 2008-2010 resampling study (28 different pesticides). Thirteen pesticides were detected during both studies. The change in the number of pesticides detected (without regard to which pesticide was detected) in groundwater samples from each of the 52 wells was evaluated and the number of pesticides detected in groundwater did not change for most of the wells (32). Of those that did have a difference between the two studies, 17 wells had more pesticide detections in groundwater during the 1995-2006 baseline study, whereas only 3 wells had more detections during the 2008-2010 resampling study.

The difference in pesticide concentrations in groundwater samples from each of the 52 wells was determined. Few changes in concentration between the 1995-2006 baseline study and the 2008-2010 resampling study were seen for most detected pesticides. Seven pesticides had a greater concentration detected in the groundwater from the same well during the baseline sampling compared to the resampling study. Concentrations of prometon, which was detected in 17 wells, were greater in the baseline study sample compared to the resampling study sample from the same well 100 percent of the time.

The change in the number of pesticides detected (without regard to which pesticide was detected) in groundwater samples from each of the 52 wells with respect to land use and geographic area was calculated. All wells with land use classified as agricultural had the same or a smaller number of pesticides detected in the resampling study compared to the baseline study. All wells in the Bighorn Basin geographic area also had the same or a smaller number of pesticides detected in the resampling study compared to the baseline study.

\section{Introduction}

In October 1991, the U.S. Environmental Protection Agency (USEPA) completed the "Pesticides and GroundWater Strategy" that describes, in part, a Federal-State partnership approach to address potential risks posed to groundwater by the use of pesticides (Wyoming Ground-water and Pesticides Strategy Committee, 1999). In response, the State of Wyoming created the Ground-water and Pesticide Strategy 
Committee (GPSC) consisting of members of local, State, and Federal government, as well as industry and interest groups, to prepare the State of Wyoming Generic Management Plan for Pesticides in Ground Water (known as the "State Management Plan" (SMP) (Wyoming Ground-water and Pesticides Strategy Committee, 1999). The SMP includes information describing individuals and organizations involved with implementation of the SMP, groundwater contamination prevention, groundwater monitoring, and required responses if pesticides are detected in groundwater.

In Wyoming, little existing information was available describing pesticide occurrence in groundwater. In accordance with the SMP, the GPSC began a program to conduct baseline groundwater sampling to characterize pesticide occurrence in Wyoming's groundwater. In addition, the GPSC identified 20 pesticides (18 parent pesticides and 2 degradates, defined as "focal compounds" and referred to in the present report as "focal pesticides") as being of greatest interest during baseline groundwater sampling).

The U.S. Geological Survey (USGS), in cooperation with the Wyoming Department of Agriculture (WDA), acting on behalf of the GPSC, later with assistance from the Wyoming Department of Environmental Quality (WDEQ), began statewide implementation of this baseline groundwater sampling in 1995. Water samples were collected during fall and spring from 296 wells in Wyoming during 1995-2006 and analyzed for pesticides. Bartos and others (2009) published a report summarizing the occurrence of pesticides in the State of Wyoming, and statistically evaluated the occurrence of pesticides in relation to selected natural and anthropogenic (humanrelated) characteristics such as geography, soils, hydrogeology, selected water-quality constituents and characteristics, and land use.

During 2008-10, the USGS, in cooperation with the WDA, resampled a subset of 52 of the 296 wells that had been sampled during the 1995-2006 baseline study. The purpose of the 2008-10 resampling was to compare detected compounds and their respective concentrations between the two sampling periods, and evaluate the occurrence of new compounds. The 52 resampled wells were distributed similarly to wells used in the 1995-2006 baseline study with respect to geographic area and land use within the geographic area of interest. In order to help evaluate changes in pesticide occurrence, wells with pesticide detections during the baseline study were given resampling priority.

\section{Purpose and Scope}

The purpose of this report is to (1) summarize the occurrence of pesticides in Wyoming groundwater during 2008-10 resampling study, (2) compare the pesticides detections and concentrations during the 2008-10 resampling study with the detections and concentrations found during the 1995-2006 baseline study, and (3) compare relations between pesticide occurrence in groundwater in 2008-10 resampling study and selected natural and anthropogenic factors. The summary of pesticide occurrence includes detection frequencies; detections by action, class, and individual pesticides; concentrations and comparisons to USEPA standards and health advisories; and detections in relation to pesticide use. Natural and anthropogenic factors were evaluated for relations with pesticide occurrence including geographic area and land use, as defined in Bartos and others (2009).

\section{Methods of Investigation}

Published methods for the selection of sampling locations, collection of groundwater samples and analysis for pesticides using USGS laboratory analytical methods and reporting conventions, and the collection and analysis of quality-control samples as part of a quality-assurance program were used in this study. The approach used to censor data and assemble final datasets was the same as the 1995-2006 baseline study (Bartos and others, 2009).

\section{Selection of Sampling Locations}

Fifty-one of the 52 wells sampled during the 2008-10 resampling study are a subset of the 296 wells sampled during the 1995-2006 baseline study (figs. 1 and 2; appendix 1). Because access to one well located in the Bighorn Basin geographic area was denied, a neighbor's well with similar depth and the same land use was used for the 2008-10 resampling study. Bartos and others (2009) described the site selection process for the 1995-2006 baseline study, including determination of geographic areas and land use. Land use within a 500-m (1,640-ft) radius surrounding each sampled well was classified using an enhanced version of the USGS 1992 National Land Cover Data (NLCD) set (Nakagaki and Wolock, 2005). The NLCD classified land use for each 30-by30-m area of the United States. Individual land uses classified within the 500-m radius then were used to classify land use into one of four predominant land-use categories using the following criteria: (1) agricultural [greater than (>) 50 percent agricultural land and less than or equal to $(\leq) 5$ percent urban land]; (2) urban ( $>25$ percent urban land use and $\leq 25$ percent agricultural land); (3) rangeland/undeveloped ( $\leq 5$ percent urban land and $\leq 25$ percent agricultural land); and (4) mixed (all other combinations of urban, agricultural, and rangeland/ undeveloped land). Each sampled well then was assigned to one of these four land-use categories to relate pesticide detections to overlying land use.

Analysis of sample data from the 1995-2006 baseline study indicated that the frequency of pesticide detections in a well was related to the geographic area in which the well was located, and to the land use surrounding the well (Bartos and 


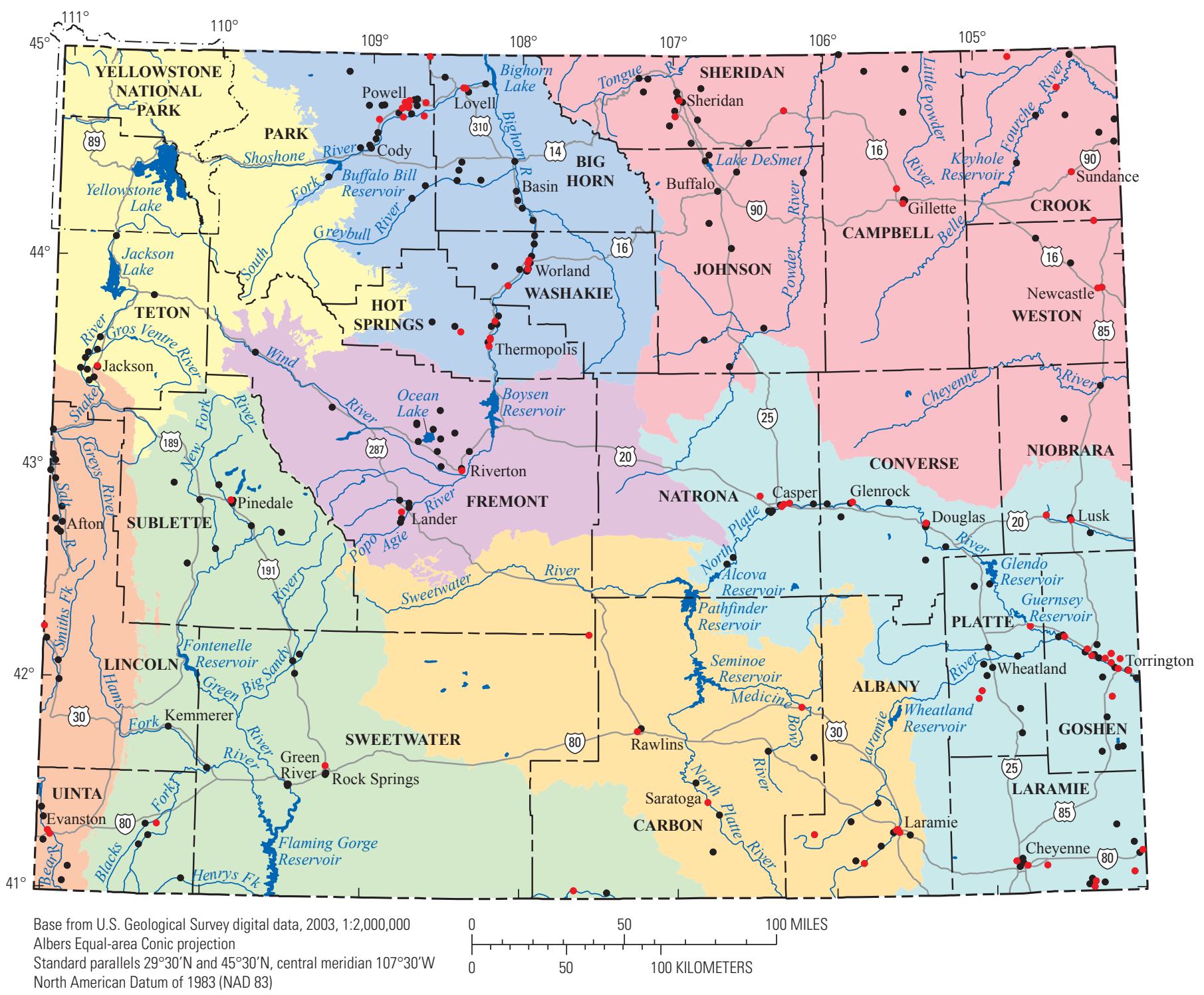

EXPLANATION

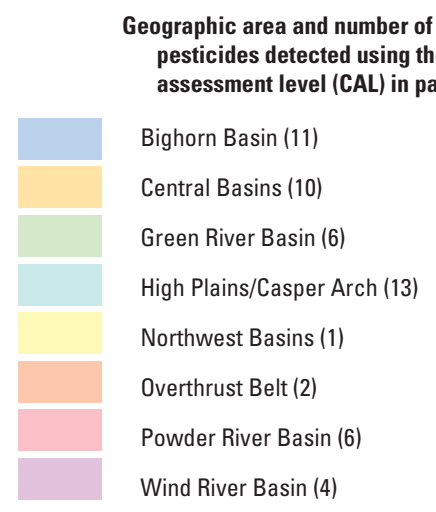

Figure 1. Wells sampled from the eight geographic areas of Wyoming during the 1995-2006 baseline study (from Bartos and others, 2009). 


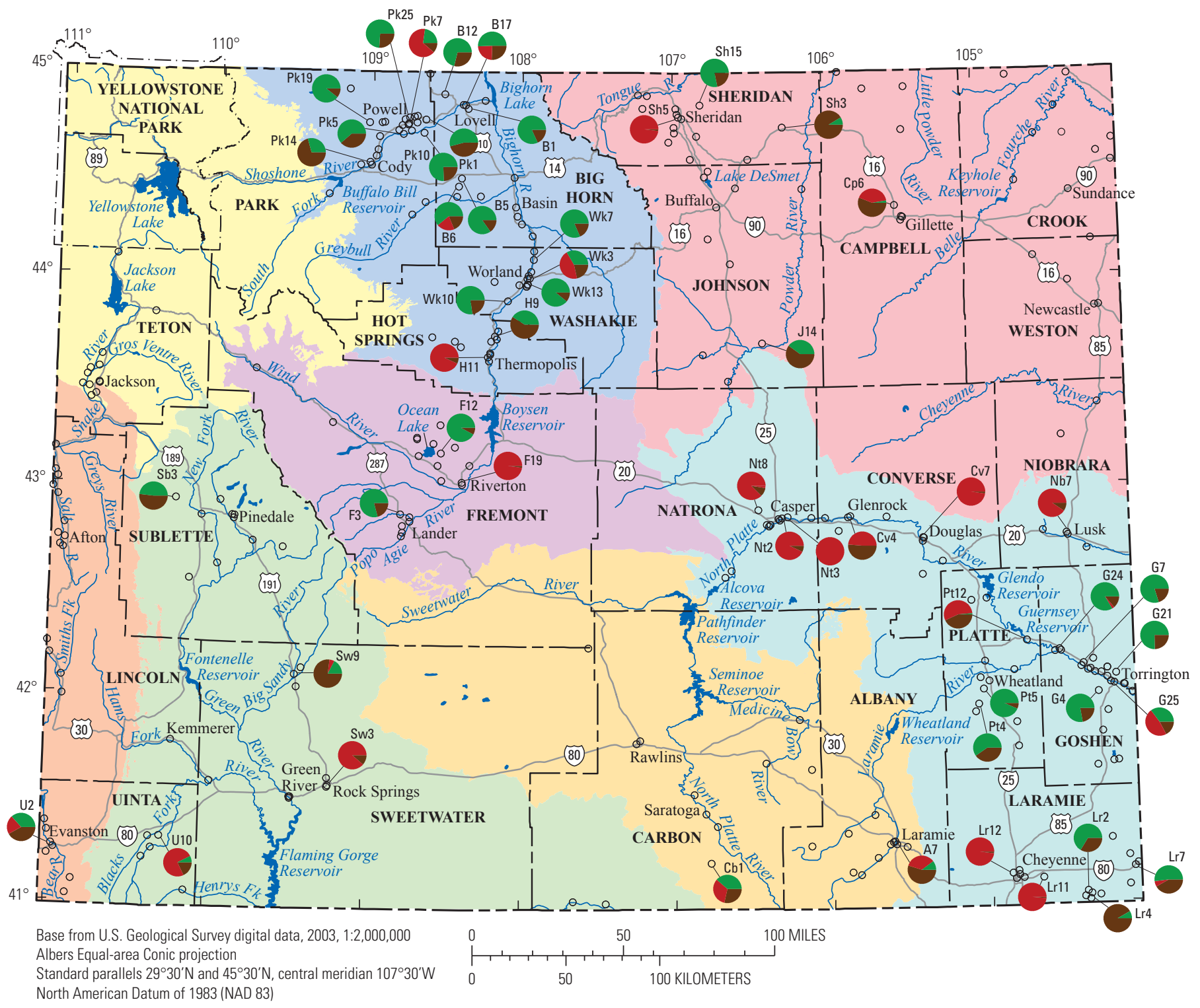

EXPLANATION

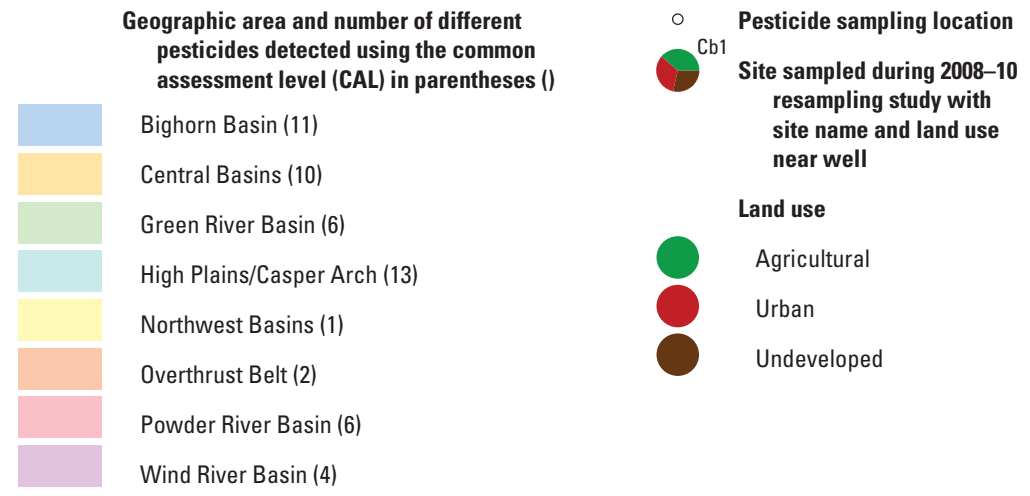

Figure 2. Location of, and land use near, the 52 wells sampled during the 2008-10 resampling study, Wyoming. 
others, 2009; Eddy-Miller and others, 2009; fig. 2). Therefore, preference for the 2008-10 resampling was given to the two geographic areas in the baseline study having the highest detection frequencies; the High Plains/Casper Arch and Bighorn Basin, which were resampled in 2008 (19 wells sampled) and 2009 (18 wells sampled), respectively. Samples from the rest of the State (15 wells) were collected during 2010. Because land use also affected the frequency of pesticide detections in a well, 2008-10 resampling sites within each of the eight geographic areas were selected to represent a similar distribution of land use; that is, a similar percentage of wells in each of the four land-use categories was selected for 2008-10 resampling compared to the 1995-2006 baseline sampling.

Because the 1995-2006 baseline study also showed that a larger number of different pesticides were detected in the fall (August-October) than in the spring (March-May), samples were collected in the fall for all three years of the 2008-10 resampling study.

\section{Sample Collection and Chemical Analyses}

During the 2008-10 resampling study, groundwater samples were collected and processed in a mobile waterquality laboratory using procedures specified by the USGS National Water-Quality Assessment Program (Koterba and others, 1995; Koterba, 1998) and by the USGS National Field Manual for the Collection of Water-Quality Data (U.S. Geological Survey, variously dated). Samples were sent to the USGS National Water Quality Laboratory (NWQL) in Denver, Colo., for analysis using analytical methods selected to detect the 19 pesticides (17 parent pesticides and 2 pesticide degradates) identified in the SMP as focal pesticides of greatest concern (table 1). (Analysis of one focal pesticide, difenzoquat, was not made, as analytical methods were not available.) The USGS NWQL analytical methods selected to detect focal pesticides in groundwater samples also could detect many additional nonfocal (as many as 136) pesticides (table 1) (Furlong and others, 2001; Madsen and others, 2003; Sandstrom and others, 2001; Zaugg and others, 1995). Additionally during the 2008-10 resampling study, samples were collected and sent to the U.S. Geological Survey Organic Chemistry Research Laboratory in Lawrence, Kansas (KSOCRL) for analysis of glyphosate and its degradates using methods described in Meyer and others (2009).

\section{Data Reporting and Treatment}

Groundwater samples were analyzed using USGS analytical methods with laboratory reporting levels much smaller than typically used in routine pesticide monitoring of public drinking-water supplies. Consequently, it is likely that much more frequent rates of detection were obtained than would have been possible with less sensitive analytical methods. Reporting levels during the 1995-2006 baseline study varied by pesticide, and many reporting levels changed during the 12 years of the baseline study. Therefore, Bartos and others (2009) recensored pesticide detections to two different assessment levels to account for (1) variations in reporting levels for the same compound and (2) variations in reporting levels among the different compounds. The assessment levels were determined for all analytical results before data were summarized and analyzed.

\section{Laboratory Reporting Levels}

During the 1995-2006 baseline study (Bartos and others, 2009) and the 2008-10 resampling study, the USGS NWQL reported analytical results for pesticides relative to three types of reporting levels, referred to as "original NWQL censoring." Very small concentrations are censored and reported as "less than" values by the NWQL to avoid false-positive detections (reporting detections when the analytes are not actually present in the sample). Censoring levels, generally known as "reporting levels," are specific to analytical methods and can change over time as methods change. During analysis of the 1995-2006 baseline data, extensive evaluation of the three types of reporting levels was performed and documented (Bartos and others, 2009). The three types of reporting levels used by the NWQL during 1995-2006 included (1) the oldest and most basic of the three types, the minimum reporting level (MRL), which is defined as the minimum concentration of a constituent that can be reliably measured using a given analytical method (Timme, 1995); (2) the method detection limit (MDL), which is defined as the minimum concentration of a constituent that can be identified, measured, and reported with 99-percent confidence to be significantly greater than zero (Childress and others, 1999); and (3) the laboratory reporting level (LRL), a reporting level used to minimize both falsepositive and false-negative detections (reporting a nondetection when the actual concentration in the sample is greater than the MDL; Childress and others, 1999). The reporting level during the 2008-10 resampling study was the LRL.

NWQL also calculates a long-term method detection level (LT-MDL), which is determined from the standard deviation of long-term laboratory spike samples (Bartos and others, 2009). NWQL qualifies detections less than the LRL, but greater than the LT-MDL as an estimated or "E" value. For this study, detections qualified with an "E" remark code were treated as quantified values. This approach preserves all of the information provided by the laboratory and acknowledges that values with an "E" remark code have concentrations greater than true nondetections. 
Table 1. Pesticides analyzed in 1995-2006 baseline and 2008-10 resampling studies, with trade names, pesticide actions, pesticide classes, laboratory reporting levels, and U.S. Environmental Protection Agency drinking-water standards.

[Pesticides detected during 2008-10 resampling study are in bold type. Focal pesticides, as determined by the Ground-water and Pesticides Strategy Committee, are shown with a grey background. USGS, U.S. Geological Survey; $\mu \mathrm{g} / \mathrm{L}$, micrograms per liter; USEPA, U.S. Environmental Protection Agency; --, not applicable; USGS analytical method: $1=$ USGS laboratory schedule 2001, $2=$ USGS laboratory schedule 2050, 3 = USGS laboratory schedule 1379, 4 = USGS laboratory schedule 2060, 5 = USGS laboratory schedule 2033, $6=$ USGS Organic Chemistry Research Laboratory in Lawrence, Kansas schedule LCGY; USEPA standard or health advisory: LHA = USEPA Lifetime Health Advisory Level (U.S. Environmental Protection Agency, 2012), MCL = USEPA Maximum Contaminant Level (U.S. Environmental Protection Agency, 2012), RSD4 = USEPA Risk-Specific Dose at $10^{-4}$ Cancer Risk (U.S. Environmental Protection Agency, 2012); DCPA, dimethyl tetrachloroterephthalate; HCH, hexachlorocyclohexane; EPTC, S-ethyl dipropylthiocarbamate; MCPA, (4-Chloro-2-methylphenoxy)acetic acid; MCPB, 4-(4-chloro-2-methylphenoxy)butanoic acid; U, chemical was analyzed for, but not detected]

\begin{tabular}{|c|c|c|c|c|c|c|c|c|}
\hline \multirow{2}{*}{ Pesticide } & \multirow{2}{*}{ Trade name } & \multirow{2}{*}{ Pesticide action } & \multirow{2}{*}{$\begin{array}{l}\text { Pesticide class } \\
\text { (or parent compound } \\
\text { class) }\end{array}$} & \multirow{2}{*}{$\begin{array}{l}\text { USGS } \\
\text { laboratory } \\
\text { parameter } \\
\text { code }\end{array}$} & \multirow{2}{*}{$\begin{array}{c}\text { USGS } \\
\text { analytical } \\
\text { method (see } \\
\text { headnote) }\end{array}$} & \multicolumn{2}{|c|}{$\begin{array}{l}\text { Laboratory reporting level } \\
\qquad(\mu \mathrm{g} / \mathrm{L})\end{array}$} & \multirow{2}{*}{$\begin{array}{l}\text { USEPA stan- } \\
\text { dard or health } \\
\text { advisory } \\
(\mu \mathrm{g} / \mathrm{L})\end{array}$} \\
\hline & & & & & & Minimum & Maximum & \\
\hline 1-Naphthol & Fourrine & Insecticide & Carbamate & 49295 & 2,5 & 0.036 & 0.09 & -- \\
\hline $2,4,5-\mathrm{T}^{1}$ & $\begin{array}{l}\text { Dacamine, Emulsavert, } \\
\text { Line Rider }\end{array}$ & Herbicide & Chlorophenoxy & 39742 & 2,4 & 0.01 & 0.32 & 70 (LHA) \\
\hline 2,4-D methyl ester & -- & Herbicide & Chlorophenoxy & 50470 & 4 & 0.009 & 0.2 & -- \\
\hline 2,4-D & Dacamine, Weed-B-Gon & Herbicide & Chlorophenoxy & 39732 & 2,4 & 0.01 & 0.73 & 70 (MCL) \\
\hline $2,4-\mathrm{DB}$ & Butoxone, Butyrac & Selective herbicide & Chlorophenoxy & 38746 & 2,4 & 0.01 & 0.25 & -- \\
\hline 2,6-Diethylaniline & Alachlor degradate & -- & Amide/acetanilide & 82660 & 1,5 & 0.002 & 0.006 & -- \\
\hline $\begin{array}{l}\text { 2-Chloro-2',6'-diethyl- } \\
\text { acetanilide }\end{array}$ & Butenachlor degradate & -- & Amide & 61618 & 5 & 0.005 & 0.01 & -- \\
\hline $\begin{array}{l}\text { 2-Chloro-4-isopropyl- } \\
\text { amino-6-amino-s- } \\
\text { triazine (CIAT) }\end{array}$ & Atrazine degradate & -- & Triazine & 04040 & $1,3,4,5$ & 0.002 & 0.06 & -- \\
\hline $\begin{array}{l}\text { 2-Chloro-6-ethylamino- } \\
\text { 4-amino-s-triazine } \\
\text { (CEAT) }\end{array}$ & $\begin{array}{l}\text { Atrazine/cyanazine/Sima- } \\
\text { zine degradate }\end{array}$ & -- & Triazine & 04038 & 3,4 & 0.01 & 0.08 & -- \\
\hline 2-Ethyl-6-methylaniline & Metolachlor degradate & -- & Amide & 61620 & 5 & 0.004 & 0.01 & -- \\
\hline $\begin{array}{l}\text { 2-Hydroxy-4-isopropyl- } \\
\text { amino-6-ethylamino-s- } \\
\text { triazine (OIET) }\end{array}$ & Atrazine degradate & -- & Triazine & 50355 & 4 & 0.008 & 0.06 & -- \\
\hline $\begin{array}{l}\text { 2-Methyl-4,6-dinitrophenol } \\
\text { (DNOC) }^{1}\end{array}$ & Dinitro-o-cresol, Elgetol & Herbicide & Miscellaneous & 49299 & 2 & 0.01 & 0.42 & -- \\
\hline 3,4-Dichloroaniline & Propanil degradate & -- & Urea & 61625 & 5 & 0.004 & 0.006 & -- \\
\hline 3,5-Dichloroaniline & Iprodione degradate & -- & Miscellaneous & 61627 & 5 & 0.004 & 0.012 & -- \\
\hline 3-Hydroxycarbofuran & Carbofuran degradate & -- & Carbamate & 49308 & 2,4 & 0.006 & 0.57 & -- \\
\hline 3-keto-Carbofuran ${ }^{1}$ & Carbofuran degradate & -- & Carbamate & 50295 & 4 & 0.01 & 0.02 & -- \\
\hline 4-Chloro-2-methylphenol & MCPA degradate & -- & Urea & 61633 & 5 & 0.005 & 0.008 & -- \\
\hline
\end{tabular}


Table 1. Pesticides analyzed in 1995-2006 baseline and 2008-10 resampling studies, with trade names, pesticide actions, pesticide classes, laboratory reporting levels, and U.S. Environmental Protection Agency drinking-water standards.-Continued

[Pesticides detected during 2008-10 resampling study are in bold type. Focal pesticides, as determined by the Ground-water and Pesticides Strategy Committee, are shown with a grey background. USGS, U.S. Geological Survey; $\mu \mathrm{g} / \mathrm{L}$, micrograms per liter; USEPA, U.S. Environmental Protection Agency; --, not applicable; USGS analytical method: $1=$ USGS laboratory schedule 2001, $2=$ USGS laboratory schedule 2050, 3 = USGS laboratory schedule 1379, 4 = USGS laboratory schedule 2060, 5 = USGS laboratory schedule 2033, $6=$ USGS Organic Chemistry Research Laboratory in Lawrence, Kansas schedule LCGY; USEPA standard or health advisory: LHA = USEPA Lifetime Health Advisory Level (U.S. Environmental Protection Agency, 2012), MCL = USEPA Maximum Contaminant Level (U.S. Environmental Protection Agency, 2012), RSD4 = USEPA Risk-Specific Dose at $10^{-4}$ Cancer Risk (U.S. Environmental Protection Agency, 2012); DCPA, dimethyl tetrachloroterephthalate; HCH, hexachlorocyclohexane; EPTC, S-ethyl dipropylthiocarbamate; MCPA, (4-Chloro-2-methylphenoxy)acetic acid; MCPB, 4-(4-chloro-2-methylphenoxy)butanoic acid; U, chemical was analyzed for, but not detected]

\begin{tabular}{|c|c|c|c|c|c|c|c|c|}
\hline \multirow{2}{*}{ Pesticide } & \multirow{2}{*}{ Trade name } & \multirow{2}{*}{ Pesticide action } & \multirow{2}{*}{$\begin{array}{c}\text { Pesticide class } \\
\text { (or parent compound } \\
\text { class) }\end{array}$} & \multirow{2}{*}{$\begin{array}{l}\text { USGS } \\
\text { laboratory } \\
\text { parameter } \\
\text { code }\end{array}$} & \multirow{2}{*}{$\begin{array}{c}\text { USGS } \\
\text { analytical } \\
\text { method (see } \\
\text { headnote) }\end{array}$} & \multicolumn{2}{|c|}{$\begin{array}{l}\text { Laboratory reporting level } \\
(\mu \mathrm{g} / \mathrm{L})\end{array}$} & \multirow{2}{*}{$\begin{array}{l}\text { USEPA stan- } \\
\text { dard or health } \\
\text { advisory } \\
(\mu \mathrm{g} / \mathrm{L})\end{array}$} \\
\hline & & & & & & Minimum & Maximum & \\
\hline Acetochlor & Guardian, Harness, Relay & Preplant herbicide & $\begin{array}{l}\text { Amide/ chloroacet- } \\
\text { amide }\end{array}$ & 49260 & $1,3,5$ & 0.002 & 0.05 & -- \\
\hline Acifluorfen & Blazer, Tackle 2S, Astic & Herbicide & Miscellaneous acid & 49315 & 2,4 & 0.007 & 0.24 & 100 (RSD4) \\
\hline Alachlor & Alanex, Lasso, Shroud & Herbicide & Amide/ acetanilide & 46342 & $1,3,5$ & 0.002 & 0.05 & 2 (MCL) \\
\hline Aldicarb & Temik & $\begin{array}{l}\text { Insecticide, nemati- } \\
\text { cide, acaricide }\end{array}$ & Carbamate & 49312 & 2,4 & 0.02 & 1.86 & $3(\mathrm{MCL})^{2}$ \\
\hline Aldicarb sulfone & $\begin{array}{l}\text { Standak, aldoxycarb, } \\
\text { aldicarb metabolite }\end{array}$ & Insecticide & Carbamate & 49313 & 2,4 & 0.02 & 1.31 & $2(\mathrm{MCL})^{2}$ \\
\hline Aldicarb sulfoxide & Aldicarb degradate & -- & Carbamate & 49314 & 2,4 & 0.008 & 0.27 & $4(\mathrm{MCL})^{2}$ \\
\hline alpha-Endosulfan & Endosulfan degradate & -- & Organochlorine & 34362 & 5 & 0.005 & 0.011 & -- \\
\hline alpha- $\mathrm{HCH}^{1}$ & & Insecticide & Organochlorine & 34253 & $1,3,5$ & 0.002 & 0.007 & -- \\
\hline Ametryn $^{1}$ & Evik & Herbicide & Triazine & 38401 & 3 & 0.05 & -- & 60 (LHA) \\
\hline $\begin{array}{l}\text { Aminomethylphosphonic } \\
\text { acid }^{3}\end{array}$ & Glyphosate degradate & -- & & 62649 & 6 & 0.02 & -- & -- \\
\hline Atrazine & Aatrex, Atranex & Herbicide & Triazine & 39632 & $1,3,4,5$ & 0.001 & 0.05 & 3 (MCL) \\
\hline Azinphos-methyl & Guthion, Crysthyon & Insecticide & Organophosphate & 82686 & 1,5 & 0.001 & 0.12 & -- \\
\hline Azinphos-methyl-oxon & & & & 61635 & & 0.12 & -- & \\
\hline Bendiocarb & Ficam, Garrox, Turcam & Insecticide & Carbamate & 50299 & 4 & 0.02 & 0.04 & -- \\
\hline Benfluralin & Balan, Benefin & Herbicide & Dinitroaniline & 82673 & 1,5 & 0.002 & 0.014 & -- \\
\hline Benomyl & Benlate, Benex & Fungicide & Carbamate & 50300 & 4 & 0.004 & 0.06 & -- \\
\hline Bensulfuron, methyl & Escuri, Londax & Herbicide & Urea/sulfonyurea & 61693 & 4 & 0.02 & 0.06 & -- \\
\hline Bentazon & Basagram, Bentzone & Herbicide & Miscellaneous & 38711 & 2,4 & 0.01 & 0.06 & 200 (LHA) \\
\hline Bromacil & Hyvar X & Herbicide & Miscellaneous & 04029 & $2,3,4$ & 0.01 & 1.1 & 70 (LHA) \\
\hline Bromoxynil & $\begin{array}{l}\text { Buctril, Brominal, Agri- } \\
\text { star }\end{array}$ & Herbicide & Miscellaneous & 49311 & 2,4 & 0.01 & 1.6 & -- \\
\hline
\end{tabular}


Table 1. Pesticides analyzed in 1995-2006 baseline and 2008-10 resampling studies, with trade names, pesticide actions, pesticide classes, laboratory reporting levels, and U.S. Environmental Protection Agency drinking-water standards.-Continued

[Pesticides detected during 2008-10 resampling study are in bold type. Focal pesticides, as determined by the Ground-water and Pesticides Strategy Committee, are shown with a grey background. USGS, U.S. Geological Survey; $\mu \mathrm{g} / \mathrm{L}$, micrograms per liter; USEPA, U.S. Environmental Protection Agency; --, not applicable; USGS analytical method: $1=$ USGS laboratory schedule 2001, $2=$ USGS laboratory schedule 2050, 3 = USGS laboratory schedule 1379, 4 = USGS laboratory schedule 2060, 5 = USGS laboratory schedule 2033, $6=$ USGS Organic Chemistry Research Laboratory in Lawrence, Kansas schedule LCGY; USEPA standard or health advisory: LHA = USEPA Lifetime Health Advisory Level (U.S. Environmental Protection Agency, 2012), MCL = USEPA Maximum Contaminant Level (U.S. Environmental Protection Agency, 2012), RSD4 = USEPA Risk-Specific Dose at 10-4 Cancer Risk (U.S. Environmental Protection Agency, 2012); DCPA, dimethyl tetrachloroterephthalate; HCH, hexachlorocyclohexane; EPTC, S-ethyl dipropylthiocarbamate; MCPA, (4-Chloro-2-methylphenoxy)acetic acid; MCPB, 4-(4-chloro-2-methylphenoxy)butanoic acid; U, chemical was analyzed for, but not detected]

\begin{tabular}{|c|c|c|c|c|c|c|c|c|}
\hline \multirow{2}{*}{ Pesticide } & \multirow{2}{*}{ Trade name } & \multirow{2}{*}{ Pesticide action } & \multirow{2}{*}{$\begin{array}{c}\text { Pesticide class } \\
\text { (or parent compound } \\
\text { class) }\end{array}$} & \multirow{2}{*}{$\begin{array}{l}\text { USGS } \\
\text { laboratory } \\
\text { parameter } \\
\text { code }\end{array}$} & \multirow{2}{*}{$\begin{array}{c}\text { USGS } \\
\text { analytical } \\
\text { method (see } \\
\text { headnote) }\end{array}$} & \multicolumn{2}{|c|}{$\begin{array}{l}\text { Laboratory reporting level } \\
\text { ( } \mu \mathrm{g} / \mathrm{L})\end{array}$} & \multirow{2}{*}{$\begin{array}{l}\text { USEPA stan- } \\
\text { dard or health } \\
\text { advisory } \\
(\mu \mathrm{g} / \mathrm{L})\end{array}$} \\
\hline & & & & & & Minimum & Maximum & \\
\hline Butachlor $^{1}$ & $\begin{array}{l}\text { Butanex, Lambast, } \\
\text { Machete }\end{array}$ & Herbicide & Amide & 04026 & 3 & 0.05 & -- & -- \\
\hline Butylate $^{1}$ & Sutan+, Genate Plus & Herbicide & Carbamate & 04028 & 1,3 & 0.002 & 0.05 & 400 (LHA) \\
\hline Carbaryl & Carbatox, Sevin & Insecticide & Carbamate & 49310 & 2,4 & 0.008 & 0.08 & 4,000 (RSD4) \\
\hline Carbaryl & Carbatox, Sevin & Insecticide & Carbamate & 82680 & 1,5 & 0.003 & 0.06 & 4,000 (RSD4) \\
\hline Carbofuran & Furadan, Futura & Insecticide & Carbamate & 49309 & 2,4 & 0.006 & 3.33 & 40 (MCL) \\
\hline Carbofuran & Furadan, Futura & Insecticide & Carbamate & 82674 & 1,5 & 0.003 & 0.06 & 40 (MCL) \\
\hline Carboxin $^{1}$ & Kisvax, Oxatin, Vitavax & Fungicide & Miscellaneous & 04027 & 3 & 0.05 & -- & 700 (LHA) \\
\hline Chloramben, methyl ester & Chloramben & Herbicide & Miscellaneous acid & 61188 & 2,4 & 0.01 & 0.42 & 100 (LHA) \\
\hline Chlorimuron & Classic, Darban, Lory & Herbicide & Urea/sulfonyurea & 50306 & 4 & 0.01 & 0.08 & -- \\
\hline $\begin{array}{l}\text { Chlorodiamino-s-triazine } \\
(\text { CAAT })^{1}\end{array}$ & Atrazine degradate & -- & Triazine & 04039 & 4 & 0.04 & -- & -- \\
\hline Chlorothalonil $^{1}$ & Bravo & Fungicide & Organochlorine & 49306 & 2,4 & 0.01 & 0.48 & 150 (RSD4) \\
\hline Chlorpyrifos & Dursban, Lorsban & Insecticide & Organophosphate & 38933 & 1,5 & 0.0036 & 0.006 & 2 (LHA) \\
\hline cis-Permethrin & Ambush, Pounce & Insecticide & Pyrethroid & 82687 & 1,5 & 0.005 & 0.016 & -- \\
\hline cis-Propiconazole & & -- & Miscellaneous & 79846 & 5 & 0.008 & 0.013 & -- \\
\hline Clopyralid & Stinger, Lontrel & Herbicide & $\begin{array}{l}\text { Pyridinecarboxylic } \\
\text { acid }\end{array}$ & 49305 & 2,4 & 0.01 & 1.82 & -- \\
\hline Cyanazine & Bladex & Selective herbicide & Triazine & 04041 & $1,3,5$ & 0.004 & 0.022 & 1 (LHA) \\
\hline Cycloate & Ro-Neet & Selective herbicide & $\begin{array}{l}\text { Carbamate/ thiocar- } \\
\text { bamate }\end{array}$ & 04031 & 3,4 & 0.01 & 0.05 & -- \\
\hline Cyfluthrin & $\begin{array}{l}\text { Aztec, Bug-b-gon, Laser, } \\
\text { Raid, Tempo }\end{array}$ & Insecticide & Pyrethroid & 61585 & 5 & 0.016 & 0.053 & -- \\
\hline Cypermethrin & Barricade, Cymbush & Insecticide & Pyrethroid & 61586 & 5 & 0.009 & 0.046 & -- \\
\hline Dacthal mono-acid & Dacthal degradate & -- & Organochlorine & 49304 & 2,4 & 0.01 & 0.15 & -- \\
\hline DCPA & Dacthal & Herbicide & Organochlorine & 82682 & 1,5 & 0.002 & 0.0076 & 70 (LHA) \\
\hline
\end{tabular}


Table 1. Pesticides analyzed in 1995-2006 baseline and 2008-10 resampling studies, with trade names, pesticide actions, pesticide classes, laboratory reporting levels, and U.S. Environmental Protection Agency drinking-water standards.-Continued

[Pesticides detected during 2008-10 resampling study are in bold type. Focal pesticides, as determined by the Ground-water and Pesticides Strategy Committee, are shown with a grey background. USGS, U.S. Geological Survey; $\mu \mathrm{g} / \mathrm{L}$, micrograms per liter; USEPA, U.S. Environmental Protection Agency; --, not applicable; USGS analytical method: $1=$ USGS laboratory schedule 2001, $2=$ USGS laboratory schedule 2050, 3 = USGS laboratory schedule 1379, 4 = USGS laboratory schedule 2060, 5 = USGS laboratory schedule 2033, $6=$ USGS Organic Chemistry Research Laboratory in Lawrence, Kansas schedule LCGY; USEPA standard or health advisory: LHA = USEPA Lifetime Health Advisory Level (U.S. Environmental Protection Agency, 2012), MCL = USEPA Maximum Contaminant Level (U.S. Environmental Protection Agency, 2012), RSD4 = USEPA Risk-Specific Dose at 10-4 Cancer Risk (U.S. Environmental Protection Agency, 2012); DCPA, dimethyl tetrachloroterephthalate; HCH, hexachlorocyclohexane; EPTC, S-ethyl dipropylthiocarbamate; MCPA, (4-Chloro-2-methylphenoxy)acetic acid; MCPB, 4-(4-chloro-2-methylphenoxy)butanoic acid; U, chemical was analyzed for, but not detected]

\begin{tabular}{|c|c|c|c|c|c|c|c|c|}
\hline \multirow{2}{*}{ Pesticide } & \multirow{2}{*}{ Trade name } & \multirow{2}{*}{ Pesticide action } & \multirow{2}{*}{$\begin{array}{c}\text { Pesticide class } \\
\text { (or parent compound } \\
\text { class) }\end{array}$} & \multirow{2}{*}{$\begin{array}{c}\text { USGS } \\
\text { laboratory } \\
\text { parameter } \\
\text { code }\end{array}$} & \multirow{2}{*}{$\begin{array}{c}\text { USGS } \\
\text { analytical } \\
\text { method (see } \\
\text { headnote) }\end{array}$} & \multicolumn{2}{|c|}{$\begin{array}{l}\text { Laboratory reporting level } \\
\qquad(\mu \mathrm{g} / \mathrm{L})\end{array}$} & \multirow{2}{*}{$\begin{array}{l}\text { USEPA stan- } \\
\text { dard or health } \\
\text { advisory } \\
\text { ( } \mu \mathrm{g} / \mathrm{L})\end{array}$} \\
\hline & & & & & & Minimum & Maximum & \\
\hline Desulfinylfipronil amide & Fipronil degradate & -- & Pyrazole & 62169 & 1,5 & 0.009 & 0.029 & -- \\
\hline Desulfinylfipronil & Fipronil degradate & -- & Pyrazole & 62170 & 1,5 & 0.004 & 0.012 & -- \\
\hline Diazinon & $\begin{array}{l}\text { Basudin, Spectracide, } \\
\text { Knoxout }\end{array}$ & Insecticide, nematicide & Organophosphate & 39572 & 1,5 & 0.002 & 0.008 & 1 (LHA) \\
\hline Dicamba & Banvel, Banex & Herbicide & Miscellaneous acid & 38442 & 2,4 & 0.01 & 0.35 & 4,000 (LHA) \\
\hline Dichlobenil $^{1}$ & Barrier, Casoron, Rootx & Herbicide & Organochlorine & 49303 & 2 & 0.02 & 1.2 & -- \\
\hline Dichloroprop & Weedone, Polymone & Herbicide & Chlorophenoxy & 49302 & 2,4 & 0.01 & 0.13 & -- \\
\hline Dichlorvos & DDVP, Vapona & Insecticide & Organophosphate & 38775 & 5 & 0.04 & -- & -- \\
\hline Dicrotophos & Bidrin, Penetrex & Insecticide & Organophosphate & 38454 & 5 & 0.08 & -- & -- \\
\hline Dieldrin & Panoram D-31, Octalox & Insecticide & Organochlorine & 39381 & 1,5 & 0.001 & 0.009 & 0.2 (RSD4) \\
\hline Dimethoate & Cygon, Defend, Rogor & Insecticide & Organophosphate & 82662 & 1,5 & 0.006 & 0.01 & -- \\
\hline Dinoseb & Premerge & Herbicide & Miscellaneous & 49301 & 2,4 & 0.01 & 0.21 & 7 (MCL) \\
\hline Diphenamid & Dymid, Enide & Selective herbicide & Amide & 04033 & 3,4 & 0.01 & 0.05 & 200 (LHA) \\
\hline Disulfoton sulfone & Disyston sulfone & Insecticide & Organophosphate & 61640 & 5 & 0.01 & 0.014 & -- \\
\hline Disulfoton & Di-Syston & Insecticide, acaricide & Organophosphate & 82677 & 1,5 & 0.02 & 0.06 & 0.7 (LHA) \\
\hline Diuron & Durashield, Karmex & Herbicide & Urea & 49300 & 2,4 & 0.01 & 0.42 & 200 (RSD4) \\
\hline Endosulfan Sulfate & Endosulfan degradate & -- & Organochlorine & 61590 & 5 & 0.014 & 0.022 & -- \\
\hline EPTC & Eptam, Eradicane & Herbicide & Carbamate & 82668 & 1,5 & 0.002 & 0.015 & -- \\
\hline Ethalfluralin ${ }^{1}$ & Eptam, Eradicane & Herbicide & Dinitroaniline & 82663 & 1 & 0.004 & 0.013 & -- \\
\hline Ethion monoxon & Ethion degradate & -- & Organophosphate & 61644 & 5 & 0.002 & 0.021 & -- \\
\hline Ethion & Klear-all, Rhodocide & Insecticide & Organophosphate & 82346 & 5 & 0.004 & 0.016 & -- \\
\hline Ethoprop (Ethoprophos) & Mocap, Prophos & Insecticide, nematicide & Organophosphate & 82672 & 1,5 & 0.003 & 0.016 & -- \\
\hline Fenamiphos sulfone & Fenamiphos degradate & -- & Organophosphate & 61645 & 5 & 0.049 & 0.054 & -- \\
\hline Fenamiphos sulfoxide & Fenamiphos degradate & -- & Organophosphate & 61646 & 5 & 0.04 & 0.08 & -- \\
\hline Fenamiphos & Nemacur & Insecticide & Organophosphate & 61591 & 5 & 0.03 & -- & 0.7 (LHA) \\
\hline
\end{tabular}


Table 1. Pesticides analyzed in 1995-2006 baseline and 2008-10 resampling studies, with trade names, pesticide actions, pesticide classes, laboratory reporting levels, and U.S. Environmental Protection Agency drinking-water standards.-Continued

PPesticides detected during 2008-10 resampling study are in bold type. Focal pesticides, as determined by the Ground-water and Pesticides Strategy Committee, are shown with a grey background. USGS, U.S. Geological Survey; $\mu \mathrm{g} / \mathrm{L}$, micrograms per liter; USEPA, U.S. Environmental Protection Agency; --, not applicable; USGS analytical method: $1=$ USGS laboratory schedule 2001, $2=$ USGS laboratory schedule 2050, 3 = USGS laboratory schedule 1379, 4 = USGS laboratory schedule 2060, 5 = USGS laboratory schedule 2033, $6=$ USGS Organic Chemistry Research Laboratory in Lawrence, Kansas schedule LCGY; USEPA standard or health advisory: LHA = USEPA Lifetime Health Advisory Level (U.S. Environmental Protection Agency, 2012), MCL = USEPA Maximum Contaminant Level (U.S. Environmental Protection Agency, 2012), RSD4 = USEPA Risk-Specific Dose at 10-4 Cancer Risk (U.S. Environmental Protection Agency, 2012); DCPA, dimethyl tetrachloroterephthalate; HCH, hexachlorocyclohexane; EPTC, S-ethyl dipropylthiocarbamate; MCPA, (4-Chloro-2-methylphenoxy)acetic acid; MCPB, 4-(4-chloro-2-methylphenoxy)butanoic acid; U, chemical was analyzed for, but not detected]

\begin{tabular}{|c|c|c|c|c|c|c|c|c|}
\hline \multirow{2}{*}{ Pesticide } & \multirow{2}{*}{ Trade name } & \multirow{2}{*}{ Pesticide action } & \multirow{2}{*}{$\begin{array}{c}\text { Pesticide class } \\
\text { (or parent compound } \\
\text { class) }\end{array}$} & \multirow{2}{*}{$\begin{array}{l}\text { USGS } \\
\text { laboratory } \\
\text { parameter } \\
\text { code }\end{array}$} & \multirow{2}{*}{$\begin{array}{c}\text { USGS } \\
\text { analytical } \\
\text { method (see } \\
\text { headnote) }\end{array}$} & \multicolumn{2}{|c|}{$\begin{array}{l}\text { Laboratory reporting level } \\
\text { ( } \mu \mathrm{g} / \mathrm{L})\end{array}$} & \multirow{2}{*}{$\begin{array}{l}\text { USEPA stan- } \\
\text { dard or health } \\
\text { advisory } \\
(\mu \mathrm{g} / \mathrm{L})\end{array}$} \\
\hline & & & & & & Minimum & Maximum & \\
\hline Fenuron & Fenuron & Herbicide & Urea & 49297 & 2,4 & 0.01 & 1.0 & -- \\
\hline Fipronil sulfide & Fipronil degradate & -- & Pyrazole & 62167 & 1,5 & 0.005 & 0.013 & -- \\
\hline Fipronil sulfone & Fipronil degradate & -- & Pyrazole & 62168 & 1,5 & 0.005 & 0.024 & -- \\
\hline Fipronil & $\begin{array}{l}\text { Combat, Frontline, Max- } \\
\text { force, Regent }\end{array}$ & Insecticide & Pyrazole & 62166 & 1,5 & 0.007 & 0.018 & -- \\
\hline Flumetsulam & Broadstrike, Python & Herbicide & Miscellaneous & 61694 & 4 & 0.01 & 0.08 & -- \\
\hline Fluometuron & Cotoran & Herbicide & Urea & 38811 & 2,4 & 0.01 & 0.36 & 90 (LHA) \\
\hline Fonofos & Dyfonate & Insecticide & Organophosphate & 04095 & 1,5 & 0.003 & 0.008 & 10 (LHA) \\
\hline Glufosinate $^{3}$ & Glyphosate degradate & -- & & 62721 & 6 & 0.02 & -- & -- \\
\hline Glyphosate $^{3}$ & Roundup & Herbicide & Organophosphate & 62722 & 6 & 0.02 & -- & 700 (MCL) \\
\hline Hexazinone & $\begin{array}{l}\text { Buckshot, Pronone, } \\
\text { Velpar }\end{array}$ & Herbicide & Triazine & 04025 & 3,5 & 0.012 & 0.05 & 400 (LHA) \\
\hline Imazaquin & Scepter & Herbicide & Miscellaneous & 50356 & 4 & 0.02 & 0.1 & -- \\
\hline Imazethapyr & New Path, Pursuit & Herbicide & Miscellaneous & 50407 & 4 & 0.02 & 0.08 & -- \\
\hline Imidacloprid & Admire, Provado & Insecticide & Miscellaneous & 61695 & 4 & 0.007 & 0.08 & -- \\
\hline Iprodione & Chipco, Rovral & Fungicide & Dicarboximide & 61593 & 5 & 0.014 & 0.538 & -- \\
\hline Isofenphos & Amaze, Pryfon & Insecticide & Organophosphate & 61594 & 5 & 0.003 & 0.011 & -- \\
\hline lambda-Cyhalothrin & Commodore, Icon & Insecticide & Pyrethroid & 61595 & 5 & 0.009 & 0.014 & -- \\
\hline Lindane $(\text { gamma-HCH })^{1}$ & Lindane, Isotoz & Insecticide & Organochlorine & 39341 & 1 & 0.004 & 0.011 & $0.2(\mathrm{MCL})$ \\
\hline Linuron & Linurex, Lorox & Herbicide & Urea & 38478 & 2,4 & 0.01 & 1.47 & -- \\
\hline Linuron $^{1}$ & Linurex, Lorox & Herbicide & Urea & 82666 & 1 & 0.002 & 0.039 & -- \\
\hline Malaoxon & Malathion degradate & -- & Organophosphate & 61652 & 5 & 0.022 & 0.039 & -- \\
\hline Malathion & Cythion, Malaspray & Insecticide & Organophosphate & 39532 & 1,5 & 0.005 & 0.031 & 500 (LHA) \\
\hline MCPA & Solve, MCP & Herbicide & Chlorophenoxy & 38482 & 2,4 & 0.01 & 0.20 & 30 (LHA) \\
\hline МСРВ & Butoxone M40, Thistrol & Herbicide & Chlorophenoxy & 38487 & 2,4 & 0.01 & 0.26 & -- \\
\hline
\end{tabular}


Table 1. Pesticides analyzed in 1995-2006 baseline and 2008-10 resampling studies, with trade names, pesticide actions, pesticide classes, laboratory reporting levels, and U.S. Environmental Protection Agency drinking-water standards.-Continued

[Pesticides detected during 2008-10 resampling study are in bold type. Focal pesticides, as determined by the Ground-water and Pesticides Strategy Committee, are shown with a grey background. USGS, U.S. Geological Survey; $\mu \mathrm{g} / \mathrm{L}$, micrograms per liter; USEPA, U.S. Environmental Protection Agency; --, not applicable; USGS analytical method: $1=$ USGS laboratory schedule 2001, $2=$ USGS laboratory schedule 2050, 3 = USGS laboratory schedule 1379, 4 = USGS laboratory schedule 2060, 5 = USGS laboratory schedule 2033, $6=$ USGS Organic Chemistry Research Laboratory in Lawrence, Kansas schedule LCGY; USEPA standard or health advisory: LHA = USEPA Lifetime Health Advisory Level (U.S. Environmental Protection Agency, 2012), MCL = USEPA Maximum Contaminant Level (U.S. Environmental Protection Agency, 2012), RSD4 = USEPA Risk-Specific Dose at $10^{-4}$ Cancer Risk (U.S. Environmental Protection Agency, 2012); DCPA, dimethyl tetrachloroterephthalate; HCH, hexachlorocyclohexane; EPTC, S-ethyl dipropylthiocarbamate; MCPA, (4-Chloro-2-methylphenoxy)acetic acid; MCPB, 4-(4-chloro-2-methylphenoxy)butanoic acid; U, chemical was analyzed for, but not detected]

\begin{tabular}{|c|c|c|c|c|c|c|c|c|}
\hline \multirow{2}{*}{ Pesticide } & \multirow{2}{*}{ Trade name } & \multirow{2}{*}{ Pesticide action } & \multirow{2}{*}{$\begin{array}{c}\text { Pesticide class } \\
\text { (or parent compound } \\
\text { class) }\end{array}$} & \multirow{2}{*}{$\begin{array}{l}\text { USGS } \\
\text { laboratory } \\
\text { parameter } \\
\text { code }\end{array}$} & \multirow{2}{*}{$\begin{array}{c}\text { USGS } \\
\text { analytical } \\
\text { method (see } \\
\text { headnote) }\end{array}$} & \multicolumn{2}{|c|}{$\begin{array}{l}\text { Laboratory reporting level } \\
\qquad(\mu \mathrm{g} / \mathrm{L})\end{array}$} & \multirow{2}{*}{$\begin{array}{l}\text { USEPA stan- } \\
\text { dard or health } \\
\text { advisory } \\
(\mu \mathrm{g} / \mathrm{L})\end{array}$} \\
\hline & & & & & & Minimum & Maximum & \\
\hline Metalaxyl & Apron, Ridamil, Subdue & Fungicide & Miscellaneous & 50359 & 4 & 0.01 & 0.04 & -- \\
\hline Metalaxyl & Apron, Ridamil, Subdue & Fungicide & Miscellaneous & 61596 & 5 & 0.005 & 0.018 & -- \\
\hline Methidathion & Somanil, Supracide & Insecticide & Organophosphate & 61598 & 5 & 0.006 & 0.012 & -- \\
\hline Methiocarb & Mesurol & Insecticide & Carbamate & 38501 & 2,4 & 0.008 & 1.99 & -- \\
\hline Methomyl & Lannate, Nudrin & Insecticide & Carbamate & 49296 & 2,4 & 0.004 & 1.09 & 200 (LHA) \\
\hline Methyl paraoxon & $\begin{array}{l}\text { Methyl parathion degra- } \\
\text { date }\end{array}$ & -- & Organophosphate & 61664 & 5 & 0.014 & 0.03 & -- \\
\hline Methyl parathion & Penncap-M, Paratox & Insecticide & Organophosphate & 82667 & 1,5 & 0.006 & 0.035 & 1 (LHA) \\
\hline Metolachlor & Bicep, Dual & Herbicide & Amide & 39415 & $1,3,5$ & 0.002 & 0.05 & 700 (LHA) \\
\hline Metribuzin & Lexone, Sencor & Herbicide & Triazine & 82630 & $1,3,5$ & 0.004 & 0.05 & 70 (LHA) \\
\hline Metsulfuron-methyl & Ally, Escort & Herbicide & Urea/sulfonyurea & 61697 & 4 & 0.03 & 0.14 & -- \\
\hline Molinate & Hydram, Ordram & Herbicide & Carbamate & 82671 & 1,5 & 0.002 & 0.007 & -- \\
\hline Myclobutanil & Rally, Systhane & Fungicide & Miscellaneous & 61599 & 5 & 0.008 & 0.033 & -- \\
\hline $\begin{array}{l}N \text {-(4-Chlorophenyl)- } N \text { '- } \\
\text { methylurea }\end{array}$ & Diuron degradate & -- & Urea & 61692 & 4 & 0.02 & 0.1 & -- \\
\hline Napropamide $^{1}$ & Devrinol & Herbicide & Amide & 82684 & 1 & 0.003 & 0.01 & -- \\
\hline Neburon & Granurex, Propuron & Herbicide & Urea & 49294 & 2,4 & 0.01 & 0.41 & -- \\
\hline Nicosulfuron & Accent, OneHope & Herbicide & Urea/sulfonyurea & 50364 & 4 & 0.01 & 0.32 & -- \\
\hline Norflurazon & Zorial, Solicam & Herbicide & Miscellaneous & 49293 & 2,4 & 0.02 & 0.32 & -- \\
\hline Oryzalin & Surflan & Herbicide & Dinitroaniline & 49292 & 2,4 & 0.01 & 1.25 & -- \\
\hline Oxamyl & Vydate & $\begin{array}{l}\text { Insecticide, acaricide, } \\
\text { nematicide }\end{array}$ & Carbamate & 38866 & 2,4 & 0.01 & 0.68 & 200 (MCL) \\
\hline Oxyfluorfen & Goal & Herbicide & Miscellaneous & 61600 & 3 & 0.007 & 0.017 & -- \\
\hline Parathion $^{1}$ & Alkron, Bladan, Fighter & Insecticide & Organophosphate & 39542 & 1 & 0.004 & 0.022 & -- \\
\hline Pebulate $^{1}$ & Tillam & Herbicide & Carbamate & 82669 & 1 & 0.002 & 0.009 & -- \\
\hline Pendimethalin & Prowl, Stomp & Herbicide & Dinitroaniline & 82683 & 1,5 & 0.004 & 0.012 & -- \\
\hline
\end{tabular}


Table 1. Pesticides analyzed in 1995-2006 baseline and 2008-10 resampling studies, with trade names, pesticide actions, pesticide classes, laboratory reporting levels, and U.S. Environmental Protection Agency drinking-water standards.-Continued

PPesticides detected during 2008-10 resampling study are in bold type. Focal pesticides, as determined by the Ground-water and Pesticides Strategy Committee, are shown with a grey background. USGS, U.S. Geological Survey; $\mu \mathrm{g} / \mathrm{L}$, micrograms per liter; USEPA, U.S. Environmental Protection Agency; --, not applicable; USGS analytical method: $1=$ USGS laboratory schedule 2001, $2=$ USGS laboratory schedule 2050, 3 = USGS laboratory schedule 1379, 4 = USGS laboratory schedule 2060, 5 = USGS laboratory schedule 2033, $6=$ USGS Organic Chemistry Research Laboratory in Lawrence, Kansas schedule LCGY; USEPA standard or health advisory: LHA = USEPA Lifetime Health Advisory Level (U.S. Environmental Protection Agency, 2012), MCL = USEPA Maximum Contaminant Level (U.S. Environmental Protection Agency, 2012), RSD4 = USEPA Risk-Specific Dose at 10-4 Cancer Risk (U.S. Environmental Protection Agency, 2012); DCPA, dimethyl tetrachloroterephthalate; HCH, hexachlorocyclohexane; EPTC, S-ethyl dipropylthiocarbamate; MCPA, (4-Chloro-2-methylphenoxy)acetic acid; MCPB, 4-(4-chloro-2-methylphenoxy)butanoic acid; U, chemical was analyzed for, but not detected]

\begin{tabular}{|c|c|c|c|c|c|c|c|c|}
\hline \multirow{2}{*}{ Pesticide } & \multirow{2}{*}{ Trade name } & \multirow{2}{*}{ Pesticide action } & \multirow{2}{*}{$\begin{array}{c}\text { Pesticide class } \\
\text { (or parent compound } \\
\text { class) }\end{array}$} & \multirow{2}{*}{$\begin{array}{l}\text { USGS } \\
\text { laboratory } \\
\text { parameter } \\
\text { code }\end{array}$} & \multirow{2}{*}{$\begin{array}{c}\text { USGS } \\
\text { analytical } \\
\text { method (see } \\
\text { headnote) }\end{array}$} & \multicolumn{2}{|c|}{$\begin{array}{l}\text { Laboratory reporting level } \\
(\mu \mathrm{g} / \mathrm{L})\end{array}$} & \multirow{2}{*}{$\begin{array}{l}\text { USEPA stan- } \\
\text { dard or health } \\
\text { advisory } \\
(\mu \mathrm{g} / \mathrm{L})\end{array}$} \\
\hline & & & & & & Minimum & Maximum & \\
\hline Phorate oxon & Phorate degradate & -- & Organophosphate & 61666 & 5 & 0.027 & 0.10 & -- \\
\hline Phorate & Thimet, Rampart & Insecticide & Organophosphate & 82664 & 1,5 & 0.02 & 0.10 & -- \\
\hline Phosmet oxon ${ }^{3}$ & Phosmet degradate & -- & Organophosphate & 61668 & 5 & 0.05 & 0.0511 & -- \\
\hline Phosmet $^{3}$ & Imidan, Percolate, Prolate & Insecticide & Organophosphate & 61601 & 5 & 0.008 & 0.08 & -- \\
\hline Picloram & Tordon & Herbicide & $\begin{array}{l}\text { Pyridinecarboxylic } \\
\text { acid }\end{array}$ & 49291 & 2,4 & 0.02 & 0.26 & $500(\mathrm{MCL})$ \\
\hline Prometon & Pramitol, Gesafram & Herbicide & Triazine & 04037 & $1,3,5$ & 0.01 & 0.05 & 400 (LHA) \\
\hline Prometryn & Caparol, Selectin & Herbicide & Triazine & 04036 & 3,5 & 0.005 & 0.05 & -- \\
\hline Propachlor $^{1}$ & Ramrod, Prolex & Herbicide & Amide & 04024 & 1,3 & 0.007 & 0.05 & 100 (RSD4) \\
\hline Propanil & Stamp & Herbicide & Amide & 82679 & 1,5 & 0.004 & 0.016 & -- \\
\hline Propargite & Comite, Omite & Insecticide, acaricide & Miscellaneous & 82685 & 1,5 & 0.01 & 0.06 & -- \\
\hline Propazine $^{1}$ & Milogard, Tritol & Herbicide & Triazine & 38535 & 3 & 0.05 & -- & 10 (LHA) \\
\hline Propham & Chem Hoe & Herbicide & Carbamate & 49236 & 2,4 & 0.01 & 7.9 & 100 (LHA) \\
\hline Propiconazole & Banner & Fungicide & Miscellaneous & 50471 & 4 & 0.01 & 0.038 & -- \\
\hline Propoxur & Baygone, Suncide & Fungicide & Carbamate & 38538 & 2,4 & 0.008 & 0.71 & -- \\
\hline Propyzamide (Pronamide) & Kerb & Herbicide & Amide & 82676 & 1,5 & 0.003 & 0.009 & 100 (RSD4) \\
\hline Siduron & Tupersan & Herbicide & Urea & 38548 & 4 & 0.02 & 0.04 & -- \\
\hline Silvex $^{1}(2,4,5-\mathrm{TP})$ & Silvex, Weed-B-Gon & Herbicide & Chlorophenoxy & 39762 & 2 & 0.02 & 0.25 & 50 (MCL) \\
\hline Simazine & Aquazine, Princep & Herbicide & Triazine & 04035 & $1,3,5$ & 0.005 & 0.05 & 4 (MCL) \\
\hline Simetryn $^{1}$ & Cymetrin, Gy-bon & Herbicide & Triazine & 04030 & 3 & 0.05 & -- & -- \\
\hline Sulfometruron-methyl & Oust & Herbicide & Urea/sulfonyurea & 50337 & 4 & 0.009 & 0.091 & -- \\
\hline Tebuconazole & Elite, Folicur, Raxil & Fungicide & Azole & 62852 & 5 & 0.02 & -- & -- \\
\hline Tebuthiuron & Graslan, Spike & Herbicide & Urea & 82670 & $1,4,5$ & 0.01 & 0.06 & 500 (LHA) \\
\hline Tefluthrin & Demand, Force, Karate & Insecticide & Pyrethroid & 61606 & 5 & 0.003 & 0.014 & -- \\
\hline Terbacil $^{1}$ & Sinbar, Herbicide 732 & Herbicide & Miscellaneous & 82665 & 1 & 0.007 & 0.034 & 90 (LHA) \\
\hline
\end{tabular}


Table 1. Pesticides analyzed in 1995-2006 baseline and 2008-10 resampling studies, with trade names, pesticide actions, pesticide classes, laboratory reporting levels, and U.S. Environmental Protection Agency drinking-water standards.-Continued

[Pesticides detected during 2008-10 resampling study are in bold type. Focal pesticides, as determined by the Ground-water and Pesticides Strategy Committee, are shown with a grey background. USGS, U.S. Geological Survey; $\mu \mathrm{g} / \mathrm{L}$, micrograms per liter; USEPA, U.S. Environmental Protection Agency; --, not applicable; USGS analytical method: $1=$ USGS laboratory schedule 2001, $2=$ USGS laboratory schedule 2050, 3 = USGS laboratory schedule 1379, 4 = USGS laboratory schedule 2060, 5 = USGS laboratory schedule 2033, $6=$ USGS Organic Chemistry Research Laboratory in Lawrence, Kansas schedule LCGY; USEPA standard or health advisory: LHA = USEPA Lifetime Health Advisory Level (U.S. Environmental Protection Agency, 2012), MCL = USEPA Maximum Contaminant Level (U.S. Environmental Protection Agency, 2012), RSD4 = USEPA Risk-Specific Dose at $10^{-4}$ Cancer Risk (U.S. Environmental Protection Agency, 2012); DCPA, dimethyl tetrachloroterephthalate; HCH, hexachlorocyclohexane; EPTC, S-ethyl dipropylthiocarbamate; MCPA, (4-Chloro-2-methylphenoxy)acetic acid; MCPB, 4-(4-chloro-2-methylphenoxy)butanoic acid; U, chemical was analyzed for, but not detected]

\begin{tabular}{|c|c|c|c|c|c|c|c|c|}
\hline \multirow{2}{*}{ Pesticide } & \multirow{2}{*}{ Trade name } & \multirow{2}{*}{ Pesticide action } & \multirow{2}{*}{$\begin{array}{c}\text { Pesticide class } \\
\text { (or parent compound } \\
\text { class) }\end{array}$} & \multirow{2}{*}{$\begin{array}{c}\text { USGS } \\
\text { laboratory } \\
\text { parameter } \\
\text { code }\end{array}$} & \multirow{2}{*}{$\begin{array}{c}\text { USGS } \\
\text { analytical } \\
\text { method (see } \\
\text { headnote) }\end{array}$} & \multicolumn{2}{|c|}{$\begin{array}{l}\text { Laboratory reporting level } \\
\qquad(\mu \mathrm{g} / \mathrm{L})\end{array}$} & \multirow{2}{*}{$\begin{array}{l}\text { USEPA stan- } \\
\text { dard or health } \\
\text { advisory } \\
(\mu \mathrm{g} / \mathrm{L})\end{array}$} \\
\hline & & & & & & Minimum & Maximum & \\
\hline Terbacil & Sinbar, Herbicide 732 & Herbicide & Miscellaneous & 04032 & 3,4 & 0.01 & 0.05 & 90 (LHA) \\
\hline Terbufos & Counter, Contraven & Insecticide & Organophosphate & 82675 & 1,5 & 0.01 & 0.02 & 0.4 (LHA) \\
\hline Terbuthylazine & Gardoprim & Herbicide & Triazine & 04022 & $1,3,5$ & $\mathrm{U}$ & 0.01 & -- \\
\hline Thiobencarb & Bolero, Saturn & Herbicide & Carbamate & 82681 & 1,5 & 0.002 & 0.016 & -- \\
\hline trans-Propiconazole & -- & -- & Miscellaneous & 79847 & 5 & 0.01 & 0.03 & -- \\
\hline Triallate $^{1}$ & Far-Go, Avadex BW & Herbicide & Carbamate & 82678 & 1 & 0.001 & 0.008 & -- \\
\hline Tribenuron $^{1}$ & Express, Urgent & Herbicide & Urea/sulfonyurea & 61159 & 4 & 0.009 & -- & -- \\
\hline Tribuphos & Def, Easy off-D, Folex & Herbicide & Organophosphate & 61591 & 5 & 0.004 & 0.035 & -- \\
\hline Triclopyr & Garlon & Herbicide & Chlorophenoxy & 49235 & 2,4 & 0.02 & 0.36 & -- \\
\hline Trifluralin & Treflan, Trim & Herbicide & Dinitroaniline & 82661 & 1,5 & 0.002 & 0.018 & 10 (LHA) \\
\hline Trifluralin ${ }^{1}$ & Treflan, Trim & Herbicide & Dinitroaniline & 04023 & 3 & 0.05 & -- & 10 (LHA) \\
\hline Vernolate $^{1}$ & Surpass, Vernam & Herbicide & Carbamate & 04034 & 2 & 0.05 & -- & -- \\
\hline
\end{tabular}

${ }^{1}$ Pesticide was not on analytical schedules 2008-10.

${ }^{2}$ The MCL for any combination of two or more of these three chemicals (aldicarb, aldicarb sulfoxide, and aldicarb sulfone) is $7 \mu \mathrm{g} / \mathrm{L}$.

${ }^{3}$ Pesticide was not on analytical schedules 1995-2008. 


\section{Recensoring and Assessment Levels}

Because of the use of three different types of reporting levels and variability in reporting-level values during the 1995-2006 baseline study, analytical results received from the NWQL with original censoring were recensored. The recensoring techniques and all but one value used for the data during the 1995-2006 baseline study (Bartos and others, 2009) were also used to recensor the 2008-10 resampling study data. During the 1995-2006 baseline study, recensoring was necessary to account for different types of reporting levels (MRL, MDL, or LRL) and (or) variable laboratory reporting levels either for a specific pesticide or between individual pesticides. The reporting levels typically vary over time due to changes in analytical methods, differences in laboratory equipment, equipment sensitivity, experience and skill of equipment operators, and laboratory conditions. Recensoring to a common value (referred to herein as the assessment level) allows for accurate calculation and comparison of detection frequencies and concentrations between individual pesticides or groups of pesticides with different types and values of reporting levels, as well as the use of quantitative statistical methods to examine pesticide occurrence.

Typically, recensoring is conducted by comparing concentrations of reported detections to all LRLs; all detections less than the largest LRL are recoded as nondetections, and all detections greater than the largest LRL are retained as detections at the originally reported laboratory concentrations. Unfortunately, pesticide reporting levels varied widely during the baseline study (table 1), and selection of the largest LRL for recensoring would result in many, if not most, detections being recoded as nondetections. Consequently, an approach was used to recensor the data in an attempt to retain as many pesticide detections as possible while still adhering to a rigorous and defensible procedure for assembly of the final dataset. In order to be able to compare data from the 1995-2006 baseline study to the 2008-10 resampling study, the laboratory reported pesticide concentrations from the 2008-10 resampling study were recensored in the same manner as the 1995-2006 baseline study data.

During the 1995-2006 baseline study data analyses, two levels of recensoring were developed to compare pesticidesa compound-specific assessment level (CSAL) that differed by compound, and a common assessment level (CAL) of 0.07 microgram per liter $(\mu \mathrm{g} / \mathrm{L})$ (Bartos and others, 2009). During the analysis of the 2008-10 resampling study data, the CSAL for all pesticides and the CAL were evaluated to determine if adjustments were needed based on the LRL reported by the NWQL during 2008-10. All pesticide CSALs remained the same as the level determined during the baseline study, with the exception of 2,4-D methyl ester, for which the CSAL increased from 0.009 to $0.02 \mu \mathrm{g} / \mathrm{L}$ (table 2). The CAL $(0.07 \mu \mathrm{g} / \mathrm{L})$ remained the same for the 2008-2010 resampling data as the value used during the 1995-2006 baseline study. In addition to the two levels of recensoring, no assessment level
(NAL) also was used to compare pesticide results. The NAL is the value the NWQL or the KSOCRL determined and reported for the sample.

\section{Quality-Control Samples and Quality Assurance}

In addition to collection of environmental groundwaterquality samples, three types of quality-control (QC) samples were collected as part of the overall quality-assurance (QA) program - field-blank samples, replicate samples, and fieldmatrix spike samples. The QC samples were collected, preserved, and analyzed using the same methods and equipment as for environmental samples. Collection and evaluation of QC samples, in addition to strict sample collection, processing, and analysis procedures, composed the field QA program. Results for the QC samples can be found in appendix 1.

\section{Field-Blank Samples}

Field-blank samples were collected to evaluate bias from the potential introduction of contamination to environmental samples during sample collection, sampling equipment cleaning, and laboratory analytical procedures. Specially prepared water that is certified to be free of organic constituents was used as the source-water for blank samples. No detections of pesticides were found in the one field-blank sample (site number 410817104470201 ) that was collected in conjunction with another project, using the same sampling equipment and procedures as the 2008-10 resampling study. This result indicated that decontamination procedures were adequate and that field and laboratory contamination of environmental samples by pesticides was minimal, and was consistent with results of field-blank samples collected during 1995-2006 baseline sampling.

\section{Replicate Samples}

A replicate sample (duplicate) is a sample collected immediately after the primary environmental sample to assess combined effects of sample-collection and laboratory procedures on measurement variability (precision). Two replicate samples were collected for analysis at sites 410108104223501 (Lr4) and 444603108383401 (Pk1) (appendix 2). The replicate and environmental samples had a total of 274 pairs of individual analyses, of which six pairs had detections of a pesticide in both the replicate and the environmental samples. Two sample pairs had detections in only one set of samples (either the replicate or the environmental sample); however, it should be noted that the detections in the one sample for both pesticides were estimated ("E") values at 6 and 25 percent of the LRL. All reported detections were used in analysis of the replicate data, including the values assigned an " $E$ " remark code (85 percent of reported detections). All replicate data were used without recensoring. 
Table 2. Pesticides detected in groundwater samples collected from 52 wells sampled during both 1995-2006 baseline study' and 2008-10 resampling study.

[Pesticides in bold were not included on the analytical schedules used during the 1995-2006 baseline study. NAL, no assessment level; CSAL, compoundspecific assessment level; CAL, common assessment level; $\mu \mathrm{g} / \mathrm{L}$, micrograms per liter; I, insecticide; H, herbicide; HD, herbicide degradate; FD, fungicide degradate; ID, insecticide degradate; F, fungicide; BF, detected during 1995-2006 baseline study fall sampling; BS, detected during 1995-2006 baseline study spring sampling; RF, detected during 2008-10 resampling study fall sampling; NS, not on analytical schedule]

\begin{tabular}{|c|c|c|c|c|c|c|c|c|}
\hline \multirow{2}{*}{ Pesticide } & \multirow{2}{*}{$\begin{array}{l}\text { Pesticide } \\
\text { action }\end{array}$} & \multicolumn{3}{|c|}{$\begin{array}{l}\text { Detection with original laboratory } \\
\text { censoring (NAL) }\end{array}$} & \multicolumn{2}{|c|}{$\begin{array}{l}\text { Laboratory reporting level } \\
\text { range }(\mu \mathrm{g} / \mathrm{L})\end{array}$} & \multicolumn{2}{|c|}{$\begin{array}{c}\text { Assigned assessment } \\
\text { level }(\mu \mathrm{g} / \mathrm{L})\end{array}$} \\
\hline & & $\begin{array}{l}\text { Baseline } \\
\text { spring }\end{array}$ & Baseline fall & $\begin{array}{l}\text { Resampling } \\
\text { fall }\end{array}$ & 1995-2006 & 2008-10 & CSAL & CAL \\
\hline 1-Napthol & I & -- & -- & $\mathrm{RF}$ & 0.09 & $0.036-0.04$ & 0.02 & 0.07 \\
\hline 2,4-D methyl ester & $\mathrm{H}$ & -- & $\mathrm{BF}$ & -- & $0.009-0.016$ & 0.02 & ${ }^{2} 0.02$ & 0.07 \\
\hline 2,4-D & $\mathrm{H}$ & -- & $\mathrm{BF}$ & -- & $0.01-0.73$ & 0.03 & 0.15 & 0.07 \\
\hline $\begin{array}{l}\text { 2-Chloro-4-isopropyl- } \\
\text { amino-6-amino-s- } \\
\text { triazine (CIAT) }\end{array}$ & HD & BS & $\mathrm{BF}$ & RF & $0.002-0.05$ & $0.006-.014$ & 0.05 & 0.07 \\
\hline $\begin{array}{l}\text { 2-Chloro-6-ethylamino- } \\
\text { 4-amino-s-triazine } \\
\text { (CEAT) }\end{array}$ & HD & BS & -- & RF & $0.01-0.08$ & $0.06-0.08$ & 0.04 & 0.07 \\
\hline $\begin{array}{l}\text { 2-Hydroxy-4-isopro- } \\
\text { pylamino-6-ethyl- } \\
\text { amino-s-triazine } \\
\text { (OIET) }\end{array}$ & HD & -- & -- & RF & $0.008-0.032$ & 0.003 & 0.016 & 0.07 \\
\hline 3,4-Dichloroanaline & HD & -- & -- & $\mathrm{RF}$ & 0.004 & $0.02-0.03$ & 0.004 & 0.07 \\
\hline 3,5-Dichloroaniline & FD & -- & -- & $\mathrm{RF}$ & $0.004-0.012$ & $0.003-0.003$ & 0.003 & 0.07 \\
\hline Aldicarb sulfone & ID & BS & $\mathrm{BF}$ & & $0.02-1.31$ & 0.04 & 0.11 & 0.07 \\
\hline Aldicarb sulfoxide & ID & BS & $\mathrm{BF}$ & RF & $0.008-0.27$ & 0.03 & 0.14 & 0.07 \\
\hline $\begin{array}{l}\text { Aminomethylphos- } \\
\text { phonic acid }\end{array}$ & HD & -- & -- & RF & NS & 0.02 & 0.02 & 0.07 \\
\hline Atrazine & $\mathrm{H}$ & BS & $\mathrm{BF}$ & RF & $0.001-0.05$ & 0.004 & 0.004 & 0.07 \\
\hline Benfluralin & $\mathrm{H}$ & -- & $\mathrm{BF}$ & -- & $0.002-0.013$ & 0.007 & 0.013 & 0.07 \\
\hline Bentazon & $\mathrm{H}$ & -- & -- & RF & $0.01-0.06$ & 0.03 & 0.06 & 0.07 \\
\hline Bromacil & $\mathrm{H}$ & BS & $\mathrm{BF}$ & RF & $0.01-1.1$ & $0.01-0.03$ & 0.06 & 0.07 \\
\hline Bromoxynil & $\mathrm{H}$ & -- & $\mathrm{BF}$ & -- & $0.01-1.6$ & 0.06 & 0.07 & 0.07 \\
\hline Carbaryl & I & -- & $\mathrm{BF}$ & -- & $0.003-0.046$ & $0.01-0.02$ & 0.05 & 0.07 \\
\hline Carbofuran & I & -- & $\mathrm{BF}$ & -- & $0.003-0.02$ & 0.02 & 0.02 & 0.07 \\
\hline Chlorimuron & $\mathrm{H}$ & -- & -- & -- & $0.01-0.046$ & 0.04 & 0.046 & 0.07 \\
\hline cis-Permethrin & I & -- & $\mathrm{BF}$ & -- & $0.005-0.016$ & $0.005-0.007$ & 0.016 & 0.07 \\
\hline Clopyralid & $\mathrm{H}$ & BS & $\mathrm{BF}$ & -- & $0.01-1.82$ & 0.03 & 0.26 & 0.07 \\
\hline Cyanazine & $\mathrm{H}$ & BS & $\mathrm{BF}$ & -- & $0.004-0.2$ & 0.011 & 0.013 & 0.07 \\
\hline Cypermethrin & I & -- & -- & RF & $0.009-0.046$ & $0.014-0.020$ & 0.01 & 0.07 \\
\hline DCPA & $\mathrm{H}$ & -- & -- & -- & $0.002-0.004$ & 0.004 & 0.004 & 0.07 \\
\hline Diazinon & I & BS & -- & -- & $0.002-0.008$ & 0.003 & 0.008 & 0.07 \\
\hline Dicamba & $\mathrm{H}$ & -- & -- & -- & $0.01-0.35$ & $0.02-0.03$ & 0.13 & 0.07 \\
\hline Dichloroprop & $\mathrm{H}$ & -- & -- & -- & $0.01-0.13$ & 0.02 & 0.06 & 0.07 \\
\hline Dieldrin & I & -- & $\mathrm{BF}$ & -- & $0.001-0.009$ & 0.004 & 0.008 & 0.07 \\
\hline Diuron & $\mathrm{H}$ & BS & $\mathrm{BF}$ & RF & $0.01-0.42$ & 0.02 & 0.06 & 0.07 \\
\hline Fipronil & I & -- & -- & RF & $0.004-0.012$ & $0.018-0.040$ & 0.02 & 0.07 \\
\hline Fipronil sulfide & ID & -- & $\mathrm{BF}$ & -- & $0.005-0.013$ & 0.006 & 0.006 & 0.07 \\
\hline
\end{tabular}


Table 2. Pesticides detected in groundwater samples collected from 52 wells sampled during both 1995-2006 baseline study and 2008-10 resampling study.-Continued

[Pesticides in bold were not included on the analytical schedules used during the 1995-2006 baseline study. NAL, no assessment level; CSAL, compoundspecific assessment level; CAL, common assessment level; $\mu \mathrm{g} / \mathrm{L}$, micrograms per liter; I, insecticide; H, herbicide; HD, herbicide degradate; FD, fungicide degradate; ID, insecticide degradate; F, fungicide; BF, detected during 1995-2006 baseline study fall sampling; BS, detected during 1995-2006 baseline study spring sampling; RF, detected during 2008-10 resampling study fall sampling; NS, not on analytical schedule]

\begin{tabular}{|c|c|c|c|c|c|c|c|c|}
\hline \multirow{2}{*}{ Detected pesticide } & \multirow{2}{*}{$\begin{array}{l}\text { Pesticide } \\
\text { action }\end{array}$} & \multicolumn{3}{|c|}{$\begin{array}{c}\text { Detection with original laboratory } \\
\text { censoring (NAL) }\end{array}$} & \multicolumn{2}{|c|}{$\begin{array}{c}\text { Laboratory reporting level } \\
\text { range }(\mu \mathrm{g} / \mathrm{L})\end{array}$} & \multicolumn{2}{|c|}{$\begin{array}{l}\text { Assigned assessment } \\
\text { level ( } \mu \mathrm{g} / \mathrm{L})\end{array}$} \\
\hline & & $\begin{array}{l}\text { Baseline } \\
\text { spring }\end{array}$ & Baseline fall & $\begin{array}{l}\text { Resampling } \\
\text { fall }\end{array}$ & 1995-2006 & 2008-10 & CSAL & CAL \\
\hline Fipronil sulfone & ID & -- & $\mathrm{BF}$ & -- & $0.005-0.024$ & 0.012 & 0.012 & 0.07 \\
\hline Flumetsulam & $\mathrm{H}$ & -- & -- & -- & $0.01-0.06$ & 0.03 & 0.06 & 0.07 \\
\hline Fluometuron & $\mathrm{H}$ & -- & -- & $\mathrm{RF}$ & $0.01-0.36$ & 0.04 & 0.02 & 0.07 \\
\hline Hexazinone & $\mathrm{H}$ & -- & -- & $\mathrm{RF}$ & $0.013-0.05$ & $0.005-0.007$ & 0.05 & 0.07 \\
\hline Imidacloprid & I & -- & -- & $\mathrm{RF}$ & $0.007-0.241$ & $0.03-0.1$ & 0.02 & 0.07 \\
\hline Metalaxyl & $\mathrm{F}$ & -- & -- & $\mathrm{RF}$ & $0.01-0.02$ & $0.007-0.018$ & 0.02 & 0.07 \\
\hline Metolachlor & $\mathrm{H}$ & BS & $\mathrm{BF}$ & $\mathrm{RF}$ & $0.002-0.05$ & 0.01 & 0.009 & 0.07 \\
\hline Oryzalin & $\mathrm{H}$ & -- & $\mathrm{BF}$ & -- & $0.01-1.25$ & 0.02 & 0.31 & 0.07 \\
\hline Picloram & $\mathrm{H}$ & BS & $\mathrm{BF}$ & $\mathrm{RF}$ & $0.02-0.26$ & 0.06 & 0.06 & 0.07 \\
\hline Prometon & $\mathrm{H}$ & $\mathrm{BS}$ & $\mathrm{BF}$ & $\mathrm{RF}$ & $0.01-0.05$ & 0.006 & 0.05 & 0.07 \\
\hline Simazine & $\mathrm{H}$ & BS & $\mathrm{BF}$ & $\mathrm{RF}$ & $0.005-0.05$ & 0.005 & 0.05 & 0.07 \\
\hline Suflometuron-methyl & $\mathrm{H}$ & -- & $\mathrm{BF}$ & $\mathrm{RF}$ & $0.009-0.091$ & 0.03 & 0.09 & 0.07 \\
\hline Tebuthiuron & $\mathrm{H}$ & $\mathrm{BS}$ & $\mathrm{BF}$ & $\mathrm{RF}$ & $0.01-0.026$ & $0.01-0.14$ & 0.01 & 0.07 \\
\hline Triallate & $\mathrm{H}$ & $\mathrm{BS}$ & $\mathrm{BF}$ & -- & $0.001-0.008$ & NS & 0.003 & 0.07 \\
\hline Triclopyr & $\mathrm{H}$ & BS & $\mathrm{BF}$ & -- & $0.02-0.36$ & 0.04 & 0.36 & 0.07 \\
\hline Trifluralin & $\mathrm{H}$ & -- & $\mathrm{BF}$ & -- & $0.002-0.012$ & $0.006-0.009$ & 0.012 & 0.07 \\
\hline
\end{tabular}

${ }^{1}$ Bartos and others (2009).

${ }^{2}$ The CSAL for 2,4-D methyl ester for the 2008-10 resampling study was modified from the assigned CSAL for the 1995-2006 baseline study because of a statistical change in the long-term method detection level.

The relative percentage difference (RPD) was calculated to compare the pesticide concentrations measured in both the environmental (sample1) and replicate (sample2) samples using the following equation:

$R P D=$ absolute value $\left(\frac{\text { sample } 1-\text { sample } 2}{\left(\frac{\text { sample } 1+\text { sample } 2}{2}\right)}\right) \times 100$

RPDs were not calculated for pairs where one value was reported as less than the LRL and not as an estimated value.
Evaluation of the two pairs of individual results with detections (six pesticides detected in both environmental and replicate samples) indicated five pesticide pairs had an RPD of less than 13 percent, and the remaining pesticide pair had an RPD of 29 percent. The differences generally were attributable to small concentration differences that resulted in relatively large RPDs because both environmental and replicate sample concentrations were estimated and small. These results were considered acceptable for this resampling study, generally were similar to the in-depth replicate sampling conducted during the 1995-2006 baseline study, and consequently, no detections were qualified or deleted on the basis of calculated RPDs. 


\section{Field-Matrix Spike Samples}

Field-matrix spike samples were analyzed to evaluate bias and variability from the environmental groundwater matrix or potential degradation of the constituent during sample processing, storage, and analysis. Field-matrix spike samples were collected in the same manner as the environmental samples, immediately following collection of the environmental samples. The samples then were injected with a known concentration of selected pesticides. Forty-five spike samples were collected during the 1995-2006 baseline study, and laboratory recoveries of most field-matrix spike samples ranged from 60 to 120 percent of the pesticide or degradate known to be in the sample during that time (Bartos and others, 2009). Because the same analytical methods were used during the 2008-10 resampling study, spike samples were collected at only one well, site number $41101910405130(\operatorname{Lr} 7)$ (appendix 3).

Overall recoveries of pesticides and degradates during the 2008-2010 resampling study averaged about 90 percent, which indicates a possible slight low bias in reported concentrations. Analysis of a subset of field-matrix spike data that included only pesticides and degradates detected in environmental samples showed about the same average recovery ( 93 percent). Two pesticides and degradates detected in environmental samples (1-napthol and bentazon), however, had low fieldmatrix spike recoveries indicating a general low bias (average recoveries less than 60 percent). Concentrations of aldicarb sulfoxide, fipronil, and sulfometuron-methyl may have been biased slightly high, as the calculated recoveries were 123 , 127, and 158 percent, respectively. These results were considered acceptable for this study, and consequently, no detections were qualified or deleted on the basis of poor recoveries.

\section{Pesticide 0ccurrence during 2008-10 Resampling Study}

The occurrences of pesticides in the samples collected from 52 wells during the 2008-10 resampling study are summarized and described in relation to pesticide action. Waterquality analyses for each well can be accessed at http://nwis. waterdata.usgs.gov/wy/nwis/qwdata (U.S. Geological Survey, 2012), by using the site number shown in appendix 4. In general, the NAL is used when describing any indication that the pesticide was detected. The CSAL is the level of assessment used when comparing detections of the same pesticide over time or between wells. The CAL is the level of assessment used when comparing detections that include different pesticides. In selected tables and figures, results for all assessment levels are shown for interpretive purposes.

\section{Pesticides Detected during 2008-10 Resampling Study}

Twenty-eight different pesticides were detected using NAL from the 52 wells resampled in 2008-10 (fig. 3; table 2). Concentrations of detected pesticides generally were small (less than $1 \mu \mathrm{g} / \mathrm{L}$ ). Three different pesticides (bromacil, glyphosate, and picloram) were detected at concentrations greater than $1 \mu \mathrm{g} / \mathrm{L}$ one time each (table 3; fig. 3), and in groundwater from three different wells. Bromacil was detected at $1.87 \mu \mathrm{g} / \mathrm{L}$ (table 3 ) in water from well Nt8 (fig. 2) in the High Plains/Casper Arch geographic area. Glyphosate was detected at $1.6 \mu \mathrm{g} / \mathrm{L}$ (table 3) in water from well Sh3 (fig. 2) in the Powder River Basin geographic area. Picloram was detected at $9.88 \mu \mathrm{g} / \mathrm{L}$ (table 3) in water from well Sh5 (fig. 2), also in the Powder River Basin geographic area.

The USEPA has established standards for physical properties and chemical constituents in drinking water that may have adverse effects on human health or that may cause cosmetic effects (for example, skin or tooth discoloration) or aesthetic effects (for example, color, taste, or odor). The Maximum Contaminant Level (MCL) is a legally enforceable and health-based standard and is the maximum permissible level for a constituent in drinking water that is delivered to a user of a public-water system (U.S. Environmental Protection Agency, 2012). A health advisory is a nonenforceable level that establishes acceptable constituent concentrations for different exposure periods ( 1 day, 10 days, long-term, and lifetime). A Lifetime Health Advisory Level is the concentration of a chemical that would not result in any known or anticipated adverse noncarcinogenic health effects over a lifetime of exposure (70 years) (U.S. Environmental Protection Agency, 2012). The Risk-Specific Dose is a concentration of a chemical with a specific risk level under certain exposure conditions over a lifetime (70 years) (U.S. Environmental Protection Agency, 2012). The USEPA drinking-water standards and health advisory levels serve as technical guidance to evaluate the suitability of water collected from private wells for human consumption.

Pesticide concentrations detected during the 2008-10 resampling study were evaluated by comparing the levels of these concentrations with several USEPA standards for drinking water or health advisories for finished (treated) water established under the Safe Drinking Water Act. In addition, pesticide detections were evaluated by the GPSC and compared to Wyoming groundwater-quality standards in accordance with the SMP (Wyoming Ground-water and Pesticides Strategy Committee, 1999).

All detected concentrations of pesticides during 2008-10 resampling study were lower than USEPA drinking-water standards or health advisories, where applicable (many pesticides did not have standards or advisories) (table 3). Most pesticide concentrations were at least an order of magnitude smaller 


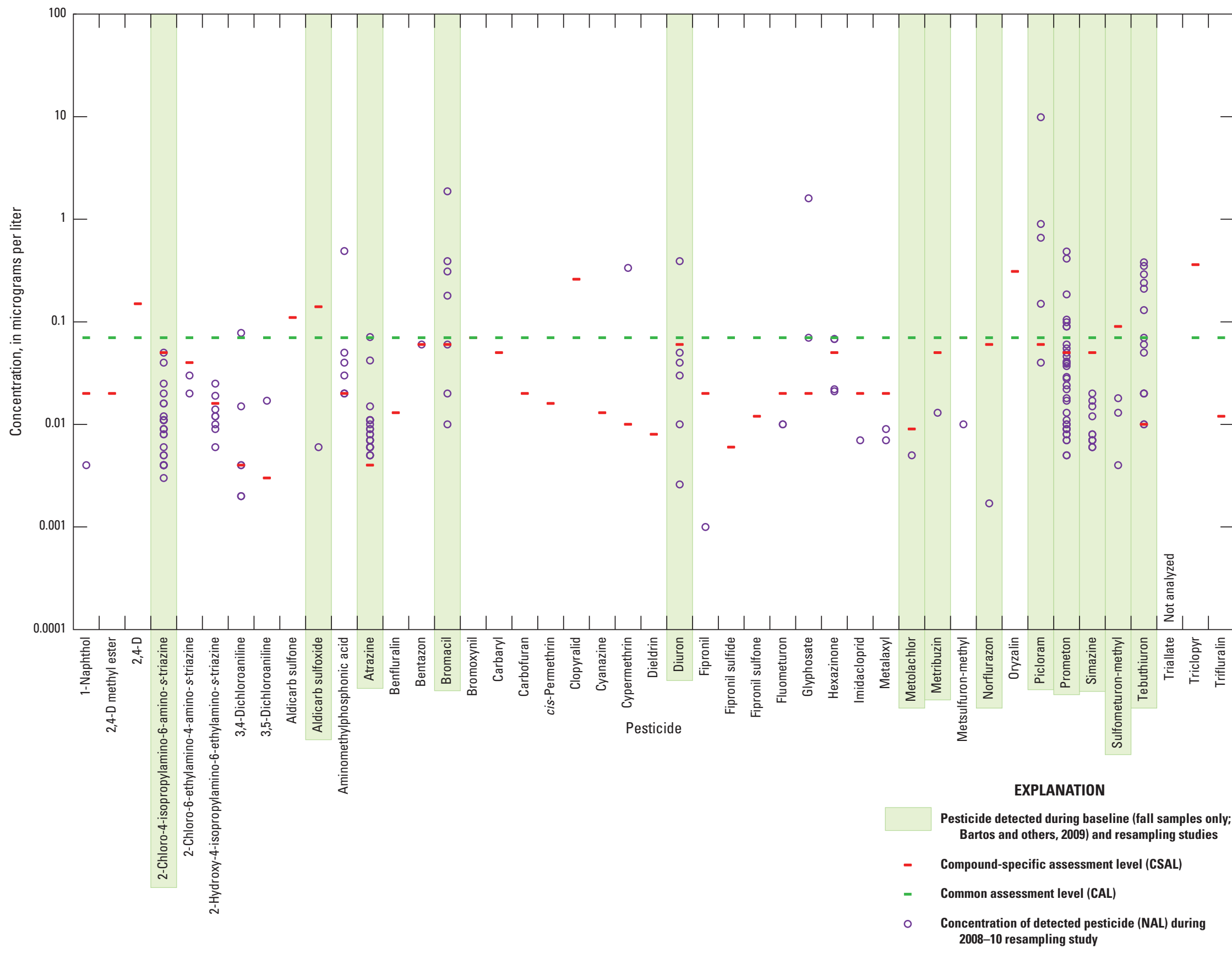

Figure 3. Pesticide detections and concentrations (using no assessment level) in groundwater samples collected from 52 wells during the 2008-10 resampling study, Wyoming. 
than USEPA drinking-water standards or health advisories. The largest pesticide or degradate concentration relative to a drinking-water standard was bromacil, at a concentration slightly less than 3 percent of the Lifetime Health Advisory Level (table 3).

\section{Pesticide Detection Frequency by Well During the 2008-10 Resampling Study}

The number of pesticides detected (pesticide detection frequencies) in groundwater from each of the 52 wells sampled during the 2008-10 resampling study was examined using the NAL, CSAL, and CAL. Pesticide detection frequencies varied, as expected, based on the assessment level used. One or more pesticides were detected at concentrations greater than the CAL in water from 16 of 52 wells sampled (about 31 percent) during the resampling study (fig. 4). As many as nine pesticides per well were detected using the NAL.

\section{Pesticide Detections by Action During the 2008-10 Resampling Study}

Detected pesticides were classified into one of six actions: herbicides, herbicide degradates, insecticides, insecticide degradates, fungicides, or fungicide degradates (fig. 5, table 2). At least 95 percent of detected pesticides were classified as herbicides or herbicide degradates regardless of whether data were examined with original censoring (NAL), or with assessment levels CSAL or CAL. Pesticides classified as insecticides, insecticide degradates, fungicides, or fungicide degradates were detected much less frequently. The prevalence of pesticides classified as herbicides was expected because herbicide use is more common than insecticide use in Wyoming, and the eight most distributed pesticides in Wyoming are herbicides (Hank Uhden, Wyoming Department of Agriculture, written commun., 2010; appendix 5).

\section{Differences in Pesticide Occurrence between 1995-2006 Baseline and 2008-10 Resampling Studies}

One purpose of the 2008-10 resampling study was to determine whether the pesticides detected in groundwater differed over time, and whether the concentrations of pesticides detected during the baseline study differed. Because the 2008-10 resampling study collected its samples only during the fall, the data from the samples collected from 52 wells were compared only to fall samples collected during the 1995-2006 baseline study. Observed changes are described in terms of pesticides (or their degradates) detected, the concentrations of detected pesticides, and the detections in relation to surrounding land use and geographic area.

\section{Differences in Pesticides Detected}

The number of different pesticides detected in samples from the 52 wells was similar between 2008-10 resampling study (28 different pesticides) (fig. 3) and the 1995-2006 baseline study (30 pesticides) (table 4; fig. 6). Thirteen pesticide detections were common to both sampling periods. Fifteen pesticides were detected during the 2008-10 resampling study that had not been detected in fall samples collected during the baseline study from the 52 wells; three of these pesticides were not on the analytical schedules used during the baseline study. Sixteen pesticides were not detected during the 2008-10 resampling study that had been detected previously during the 1995-2006 baseline study. Most of the pesticides detected in only one study, but not the other, were only detected one or two times, with the exception of hexazinone that was detected three times during the resampling study.

\section{Differences in Pesticide Detections}

The change in the number of pesticides detected (without regard to which pesticide was detected) in groundwater samples between the 1995-2006 baseline study and 2008-10 resampling study from each of the 52 sampled wells is shown in figure 7. Most of the wells (32) had no changes in the number of pesticides detected in groundwater samples. Of the wells that did have a difference between the two sampling studies, 17 wells had more pesticide detections during the 1995-2006 baseline study, whereas only 3 wells had more pesticide detections during the 2008-10 resampling study.

\section{Differences in Pesticide Concentrations}

Pesticide concentration changes in groundwater samples collected from the 52 wells was determined by evaluating pesticides with at least 50 analyses during both studies, and with at least one concentration greater than the CSAL (table 5). Most pesticides (11 of the 19 evaluated) with detections had little change in concentration between the 1995-2006 baseline study and 2008-10 resampling study. Seven pesticides generally had greater concentrations in the groundwater from the same well during the 1995-2006 baseline study than during the 2008-2010 resampling study. Concentrations of atrazine, which was detected in 33 wells, and concentrations of the atrazine degradate 2-chloro-4-isopropylamino-6-amino-straizine, were greater in the 1995-2006 baseline study sample than in the 2008-10 resampling study sample about 80 percent of the time. Concentrations of prometon, detected in 17 wells, were greater in the 1995-2006 baseline study sample than in the 2008-10 resampling study sample 100 percent of the time. Only one pesticide, diuron, detected in only four wells at concentrations greater than the CSAL, generally had greater concentrations in the 2008-10 resampling study sample than in the 1995-2006 baseline study sample. 
Table 3. Summary of pesticide detections and concentrations in groundwater sampled during the 2008-10 resampling study, Wyoming.

[Pesticides highlighted in yellow were analyzed for, but not detected during the 1995-2006 baseline study; ${ }^{1}$ pesticides highlighted in orange were not analyzed for during the 1995-2006 baseline study. ${ }^{1} \mathrm{NAL}$, no assessment level; CSAL, compound-specific assessment level; CAL, common assessment level; $\mu \mathrm{g} / \mathrm{L}$, micrograms per liter; USEPA, U.S. Environmental Protection Agency; USEPA standard or health advisory: MCL = USEPA Maximum Contaminant Level (U.S. Environmental Protection Agency, 2012), LHA = USEPA Lifetime Health Advisory Level (U.S. Environmental Protection Agency, 2012), RSD4 = USEPA RiskSpecific Dose at $10^{-4}$ Cancer Risk (U.S. Environmental Protection Agency, 2012); --, not applicable]

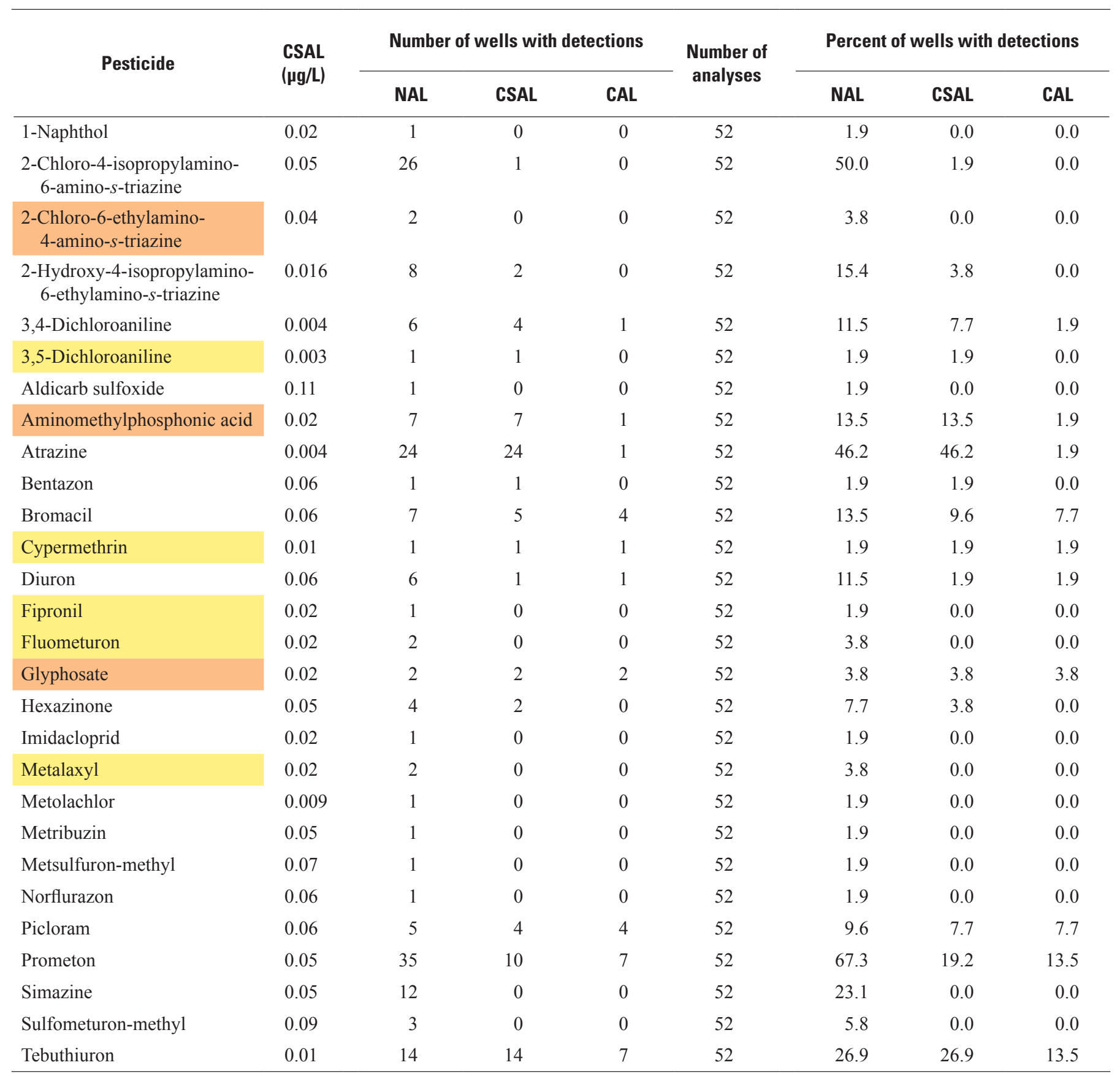

${ }^{1}$ Bartos and others (2009). 
Table 3. Summary of pesticide detections and concentrations in groundwater sampled during the 2008-10 resampling study, Wyoming.-Continued

[Pesticides highlighted in yellow were analyzed for, but not detected during the $1995-2006$ baseline study; ${ }^{1}$ pesticides highlighted in orange were not analyzed for during the 1995-2006 baseline study. ${ }^{1} \mathrm{NAL}$, no assessment level; CSAL, compound-specific assessment level; CAL, common assessment level; $\mu \mathrm{g} / \mathrm{L}$, micrograms per liter; USEPA, U.S. Environmental Protection Agency; USEPA standard or health advisory: MCL = USEPA Maximum Contaminant Level (U.S. Environmental Protection Agency, 2012), LHA = USEPA Lifetime Health Advisory Level (U.S. Environmental Protection Agency, 2012), RSD4 = USEPA RiskSpecific Dose at $10^{-4}$ Cancer Risk (U.S. Environmental Protection Agency, 2012); --, not applicable]

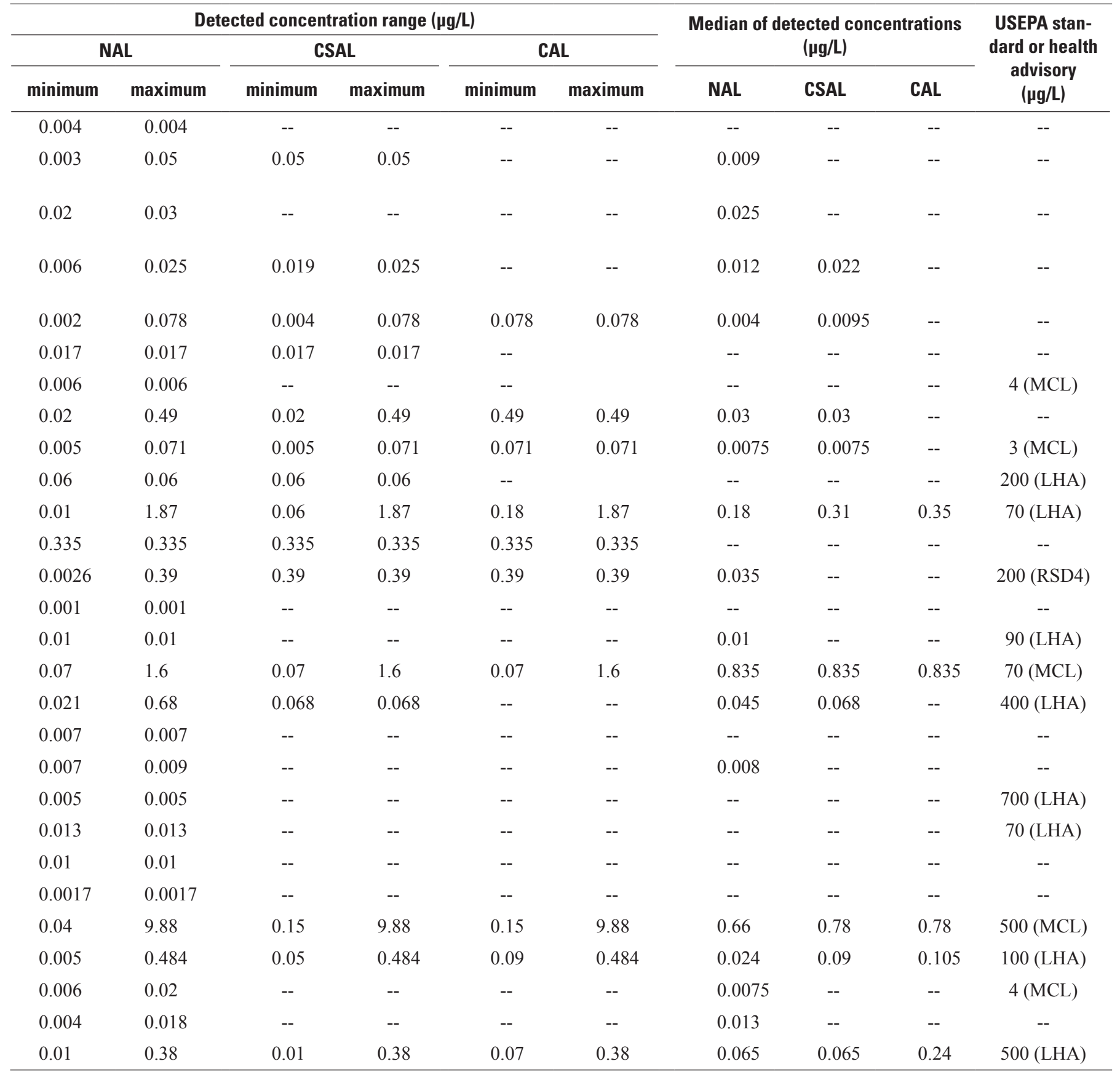




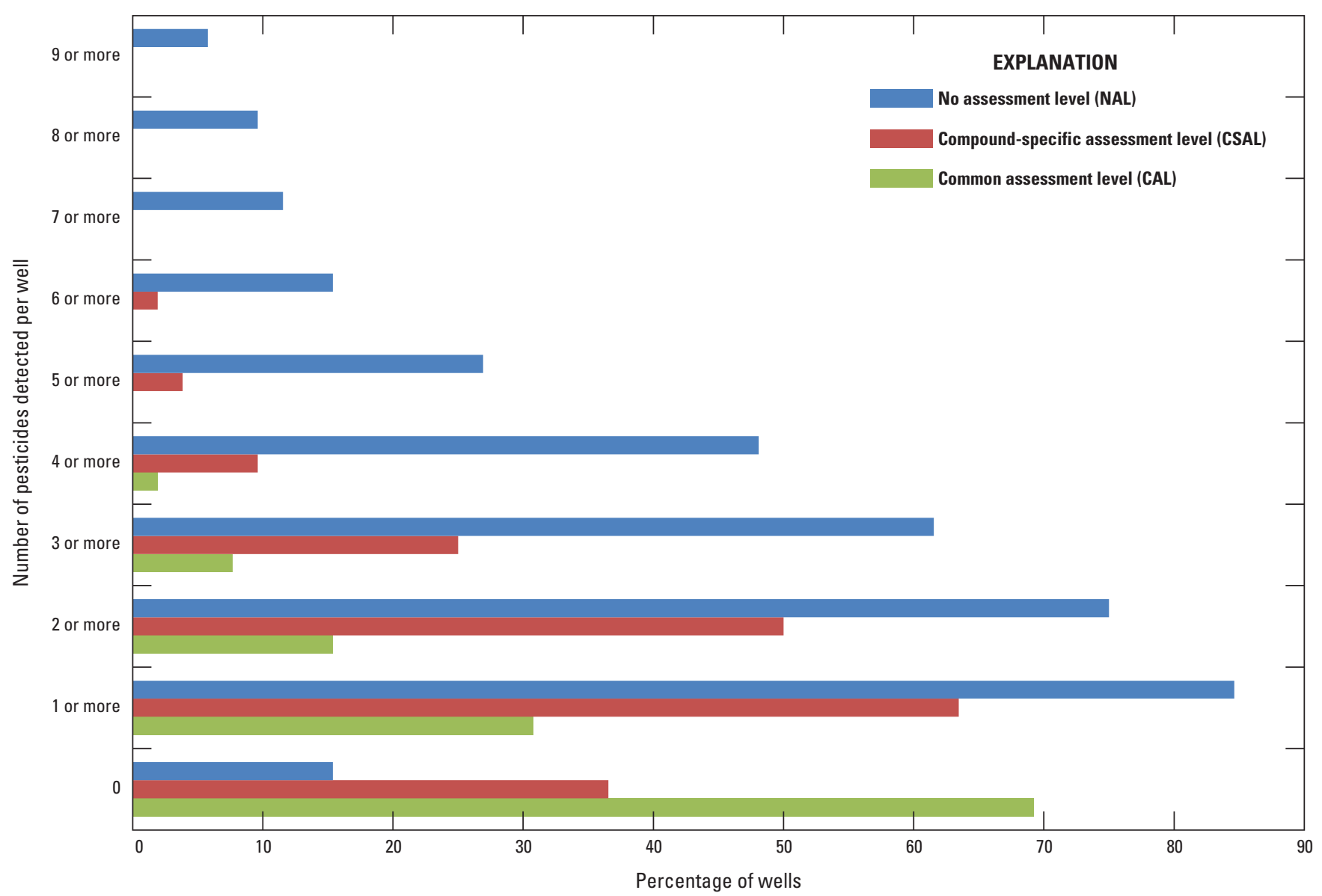

Figure 4. Number of pesticides detected in groundwater samples collected from 52 wells sampled during the 2008-10 resampling study using no assessment level, compound-specific assessment level, and common assessment level, Wyoming.

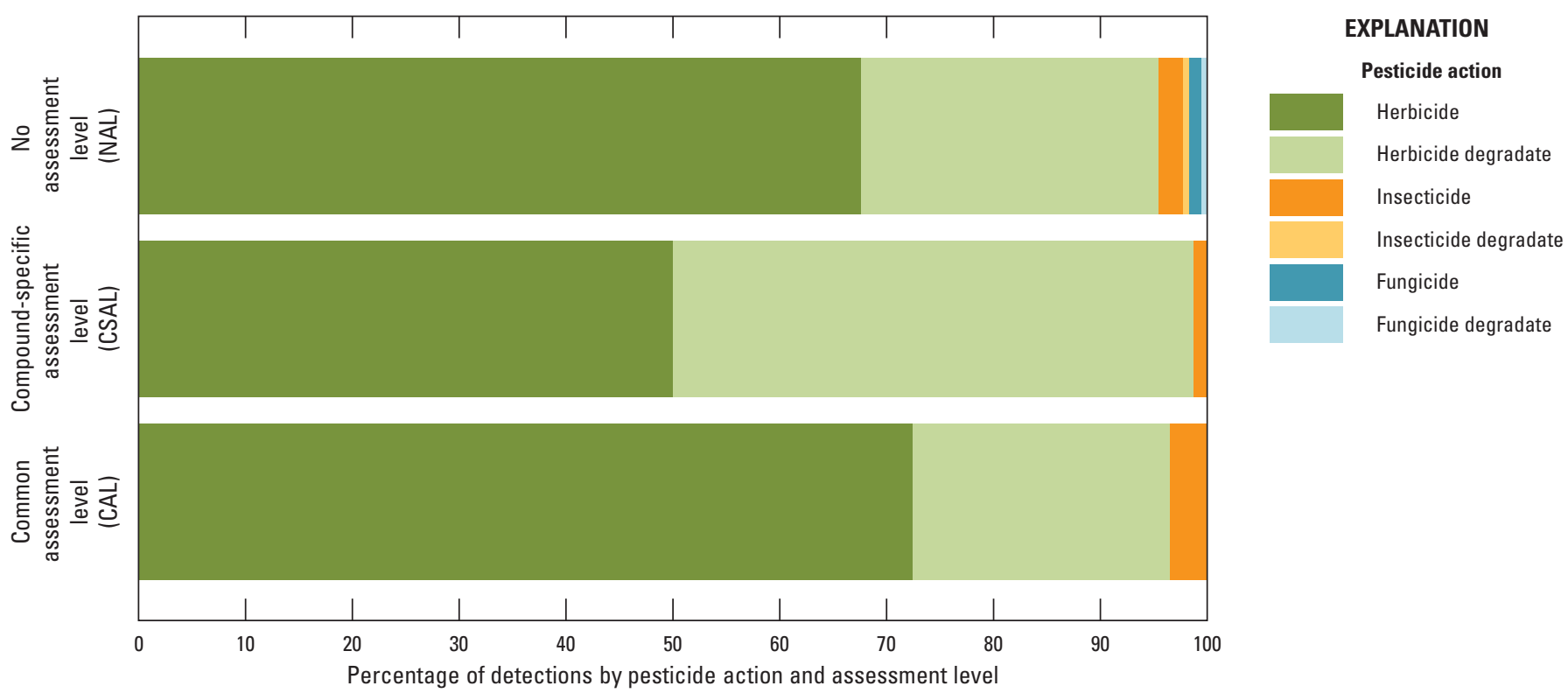

Figure 5. Pesticide detections relative to pesticide action in groundwater samples collected from 52 wells sampled during the 2008-10 resampling study, Wyoming. 
Table 4. Pesticides detected in the 52 wells sampled during 1995-2006 baseline study ${ }^{1}$ and (or) 2008-10 resampling study, Wyoming. [CSALs and CALs shown in bold indicate pesticide detected at concentration equal to or greater than the assessment level in at least one well in resampling study. NAL, no assessment level; CSAL, compound-specific assessment level; CAL, common assessment level; $\mu$ g/L, micrograms per liter; ND, not detected; D, detected; NA, not analyzed]

\begin{tabular}{|c|c|c|c|c|c|c|}
\hline \multirow[t]{2}{*}{ Pesticide } & \multicolumn{2}{|c|}{ Number of analyses } & \multicolumn{2}{|c|}{$\begin{array}{c}\text { Detection of pesticide } \\
\text { during study (NAL) }\end{array}$} & \multicolumn{2}{|c|}{$\begin{array}{c}\text { Pesticide assessment } \\
\text { level }^{1}(\mu \mathrm{g} / \mathrm{L})\end{array}$} \\
\hline & 1995-2006 & 2008-10 & 1995-2006 & 2008-10 & CSAL & CAL \\
\hline 2,4-D methyl ester & 9 & 52 & $\mathrm{D}$ & ND & 0.02 & 0.07 \\
\hline $2,4-\mathrm{D}$ & 51 & 52 & $\mathrm{D}$ & ND & 0.15 & 0.07 \\
\hline 2-Chloro-4-isopropylamino-6-amino-s-triazine & 52 & 52 & $\mathrm{D}$ & $\mathrm{D}$ & 0.05 & 0.07 \\
\hline 2-Hydroxy-4-isopropylamino-6-ethylamino-s-triazine & 8 & 52 & ND & $\mathrm{D}$ & 0.016 & 0.07 \\
\hline 3,4-Dichloroaniline & 1 & 52 & ND & $\mathrm{D}$ & 0.004 & 0.07 \\
\hline 3,5-Dichloroaniline & 1 & 52 & ND & $\mathrm{D}$ & 0.003 & 0.07 \\
\hline Aldicarb sulfone & 52 & 52 & $\mathrm{D}$ & ND & 0.11 & 0.07 \\
\hline Aldicarb sulfoxide & 51 & 52 & $\mathrm{D}$ & $\mathrm{D}$ & 0.14 & 0.07 \\
\hline Bentazon & 50 & 52 & ND & $\mathrm{D}$ & 0.06 & 0.07 \\
\hline Bromacil & 51 & 52 & $\mathrm{D}$ & $\mathrm{D}$ & 0.06 & 0.07 \\
\hline Bromoxynil & 51 & 52 & $\mathrm{D}$ & ND & 0.07 & 0.07 \\
\hline Carbaryl & 52 & 52 & $\mathrm{D}$ & ND & 0.05 & 0.07 \\
\hline Carbofuran & 52 & 52 & $\mathrm{D}$ & ND & 0.02 & 0.07 \\
\hline cis-Permethrin & 52 & 52 & $\mathrm{D}$ & ND & 0.016 & 0.07 \\
\hline Clopyralid & 51 & 52 & $\mathrm{D}$ & ND & 0.26 & 0.07 \\
\hline Cyanazine & 52 & 52 & $\mathrm{D}$ & ND & 0.013 & 0.07 \\
\hline Cypermethrin & 1 & 52 & ND & $\mathrm{D}$ & 0.01 & 0.07 \\
\hline Dieldrin & 52 & 52 & $\mathrm{D}$ & ND & 0.008 & 0.07 \\
\hline Hexazinone & 1 & 52 & ND & $\mathrm{D}$ & 0.05 & 0.07 \\
\hline Imidacloprid & 9 & 52 & ND & $\mathrm{D}$ & 0.02 & 0.07 \\
\hline Metalaxyl & 9 & 52 & ND & $\mathrm{D}$ & 0.02 & 0.07 \\
\hline Metolachlor & 52 & 52 & $\mathrm{D}$ & $\mathrm{D}$ & 0.009 & 0.07 \\
\hline Metribuzin & 52 & 52 & $\mathrm{D}$ & $\mathrm{D}$ & 0.05 & 0.07 \\
\hline Metsulfuron-methyl & 9 & 52 & ND & $\mathrm{D}$ & 0.07 & 0.07 \\
\hline Norflurazon & 51 & 52 & $\mathrm{D}$ & $\mathrm{D}$ & 0.06 & 0.07 \\
\hline Oryzalin & 51 & 52 & $\mathrm{D}$ & ND & 0.31 & 0.07 \\
\hline Picloram & 51 & 52 & $\mathrm{D}$ & $\mathrm{D}$ & 0.06 & 0.07 \\
\hline Prometon & 52 & 52 & $\mathrm{D}$ & $\mathrm{D}$ & 0.05 & 0.07 \\
\hline Simazine & 52 & 52 & $\mathrm{D}$ & $\mathrm{D}$ & 0.05 & 0.07 \\
\hline Sulfometuron-methyl & 9 & 52 & $\mathrm{D}$ & $\mathrm{D}$ & 0.09 & 0.07 \\
\hline Tebuthiuron & 52 & 52 & $\mathrm{D}$ & $\mathrm{D}$ & 0.01 & 0.07 \\
\hline Triallate & 52 & 0 & $\mathrm{D}$ & NA & 0.003 & 0.07 \\
\hline Triclopyr & 51 & 52 & $\mathrm{D}$ & ND & 0.36 & 0.07 \\
\hline Trifluralin & 52 & 52 & $\mathrm{D}$ & ND & 0.012 & 0.07 \\
\hline
\end{tabular}

${ }^{1}$ Bartos and others (2009), fall samples only. 


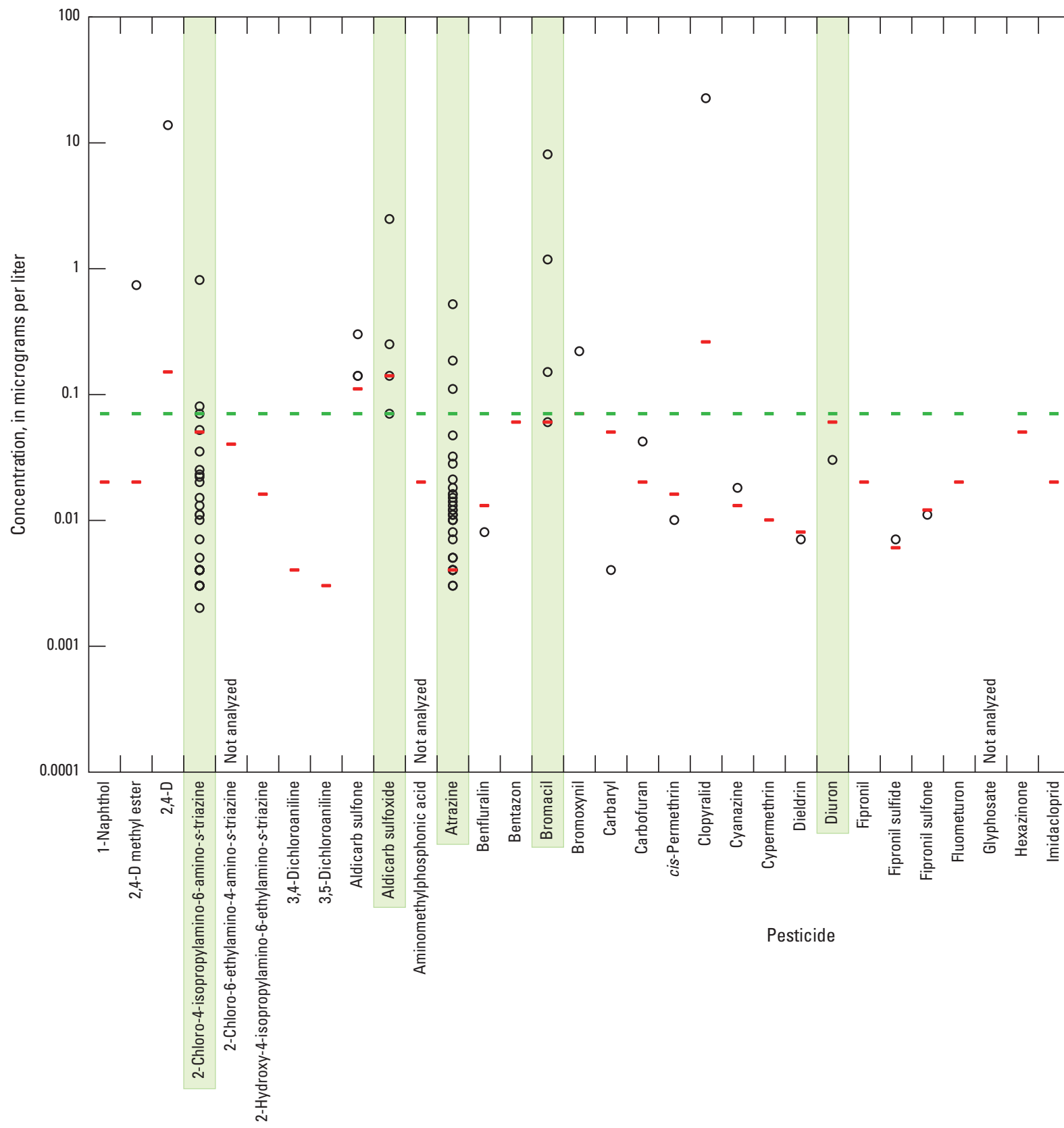

Figure 6. Pesticide detections and concentrations (using no assessment level) in groundwater samples collected from 52 wells during the $1995-2006$ baseline study (fall samples only; Bartos and others, 2009). 


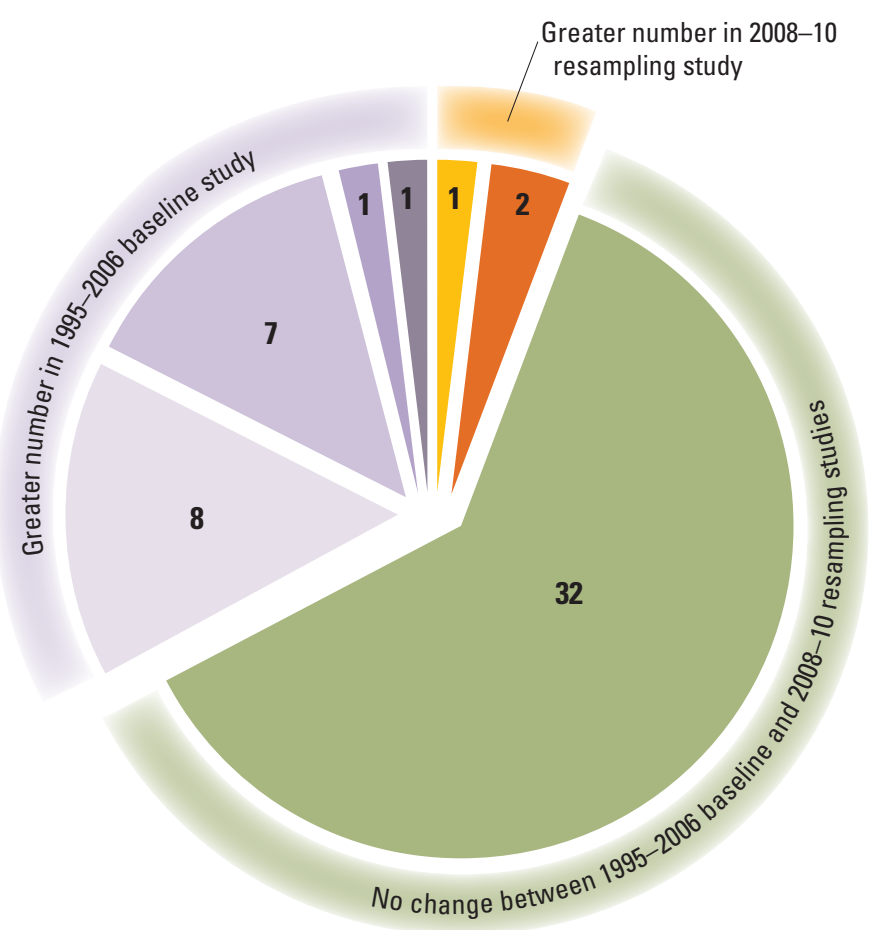

EXPLANATION

2 Number of wells in category

Changes in number of pesticides

One more resampling

Two more resampling

No difference between baseline and resampling

One more baseline

Two more baseline

Three more baseline

Four more baseline

Figure 7. Difference in number of pesticides detected in the same well between the 1995-2006 baseline (fall samples only; Bartos and others, 2009) and 2008-10 resampling studies. Pesticide detection is based on the common assessment level.

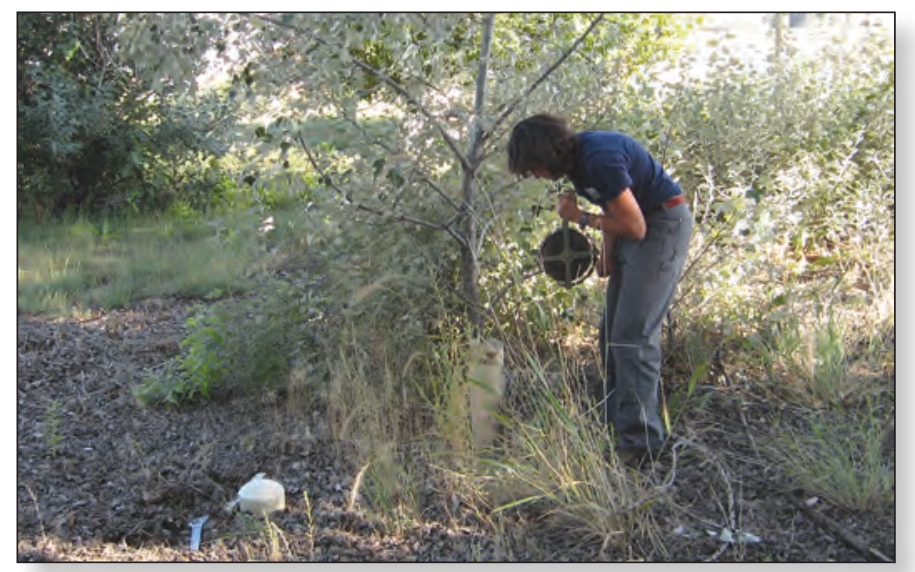

USGS hydrologist measuring the water level in a well in the Bighorn Basin geographic area. Photograph by Seth L. Davidson, September 2009.

\section{Differences in Pesticide Detections and Detection Frequency Relative to Land Use and Geographic Area}

The change in the number of pesticides detected and the detection frequency relative to land use and geographic area between the baseline and resampling studies was determined. Concentrations of pesticides detected during the baseline and resampling studies by assessment levels, including land use, are shown in figure 8 .

The overall frequency of pesticide detections in groundwater from the 52 wells was examined by calculating the percentage of wells with at least one pesticide detected at concentrations greater than the CAL with respect to land use near the well and geographic area (fig. 9). The detection frequency of at least one pesticide in groundwater from a well decreased or remained unchanged, regardless of land use or geographic area, between the 1995-2006 baseline and 2008-10 resampling studies. The detection frequency within the agricultural land-use category had the largest change from the 1995-2006 baseline study to the 2008-10 resampling study, changing from 38 percent detection frequency to zero. The detection frequency within the Bighorn Basin geographic area had the largest change from the 1995-2006 baseline study to the 2008-10 resampling study, changing from 56 percent to 17 percent.

The change in the number of pesticides detected (without regard to which pesticide was detected) between the 1995-2006 baseline study and the 2008-10 resampling study in groundwater samples from each of the 52 wells with respect to land use and geographic area was calculated and is shown figure 7 , and indicate as expected, that most wells had no change in the number of pesticides detected, regardless of land use or geographic area. This refinement shows that all wells with nearby land use classified as agricultural had the same or a smaller number of pesticides in the 2008-10 resampling study compared to the 1995-2006 baseline study. Figure 10 also shows that all wells in the Bighorn Basin geographic area had the same or a smaller number of pesticides detected in the 2008-10 resampling study compared to the 1995-2006 baseline study. in figure 10. Results shown in figure 10 are a refinement of 
Table 5. Differences in pesticide detections between the 1995-2006 baseline study ${ }^{1}$ and the 2008-10 resampling study, Wyoming. Differences were calculated on a well by well basis for pesticides with at least 50 analyses during both sampling studies and with at least one concentration greater than the compound-specific assessment level (CSAL).

[Pesticides highlighted in green indicate minimal change between baseline study and resampling study (one well or fewer with a concentration difference); pesticides highlighted in purple indicate generally greater concentrations detected in baseline study than in resampling study from same well; pesticides highlighted in tan indicate generally greater concentrations detected in resampling study than in baseline study from same well]

\begin{tabular}{|c|c|c|c|c|c|c|c|c|c|}
\hline \multirow{3}{*}{ Pesticide } & \multicolumn{2}{|c|}{ Number of analyses } & \multirow{3}{*}{$\begin{array}{c}\text { Pesticide not } \\
\text { detected above } \\
\text { CSAL in either } \\
\text { sample }\end{array}$} & \multicolumn{6}{|c|}{ Concentration greater than CSAL in at least one sample } \\
\hline & \multirow{2}{*}{$\begin{array}{l}\text { 1995-2006 } \\
\text { baseline } \\
\text { study }^{2}\end{array}$} & \multirow{2}{*}{$\begin{array}{l}2008-10 \\
\text { resampling } \\
\text { study }\end{array}$} & & \multicolumn{2}{|c|}{$\begin{array}{c}\text { Concentrations greater in } \\
\text { baseline study }\end{array}$} & \multicolumn{2}{|c|}{$\begin{array}{l}\text { Concentrations equal in baseline } \\
\text { and resampling studies }\end{array}$} & \multicolumn{2}{|c|}{$\begin{array}{l}\text { Concentrations greater in } \\
\text { resampling study }\end{array}$} \\
\hline & & & & Number of wells & Percentage & Number of wells & Percentage & Number of wells & Percentage \\
\hline $2,4-\mathrm{D}$ & 51 & 52 & 50 & 1 & 100 & 0 & 0 & 0 & 0 \\
\hline $\begin{array}{l}\text { 2-Chloro-4-isopropylamino- } \\
\text { 6-amino-s-triazine }\end{array}$ & 52 & 52 & 47 & 4 & 80 & 0 & 0 & 1 & 20 \\
\hline Aldicarb sulfone & 51 & 52 & 48 & 3 & 100 & 0 & 0 & 0 & 0 \\
\hline Aldicarb sulfoxide & 51 & 52 & 47 & 4 & 100 & 0 & 0 & 0 & 0 \\
\hline Atrazine & 52 & 52 & 19 & 25 & 76 & 1 & 3 & 7 & 21 \\
\hline Bentazon & 50 & 52 & 49 & 0 & 0 & 0 & 0 & 1 & 100 \\
\hline Bromacil & 51 & 52 & 44 & 4 & 57 & 0 & 0 & 3 & 43 \\
\hline Bromoxynil & 51 & 52 & 50 & 1 & 100 & 0 & 0 & 0 & 0 \\
\hline Carbaryl & 52 & 52 & 51 & 1 & 100 & 0 & 0 & 0 & 0 \\
\hline Carbofuran & 52 & 52 & 51 & 1 & 100 & 0 & 0 & 0 & 0 \\
\hline Clopyralid & 51 & 52 & 50 & 1 & 100 & 0 & 0 & 0 & 0 \\
\hline Cyanazine & 52 & 52 & 51 & 1 & 100 & 0 & 0 & 0 & 0 \\
\hline Diuron & 51 & 52 & 47 & 1 & 25 & 0 & 0 & 3 & 75 \\
\hline Metolachlor & 52 & 52 & 50 & 2 & 100 & 0 & 0 & 0 & 0 \\
\hline Oryzalin & 51 & 52 & 50 & 1 & 100 & 0 & 0 & 0 & 0 \\
\hline Picloram & 51 & 52 & 44 & 4 & 57 & 0 & 0 & 3 & 43 \\
\hline Prometon & 52 & 52 & 35 & 17 & 100 & 0 & 0 & 0 & 0 \\
\hline Tebuthiuron & 52 & 52 & 36 & 9 & 56 & 1 & 6 & 6 & 38 \\
\hline Triclopyr & 51 & 52 & 50 & 1 & 100 & 0 & 0 & 0 & 0 \\
\hline
\end{tabular}

${ }^{1}$ Bartos and others (2009), fall samples only.

${ }^{2}$ Number of samples used for comparison. 


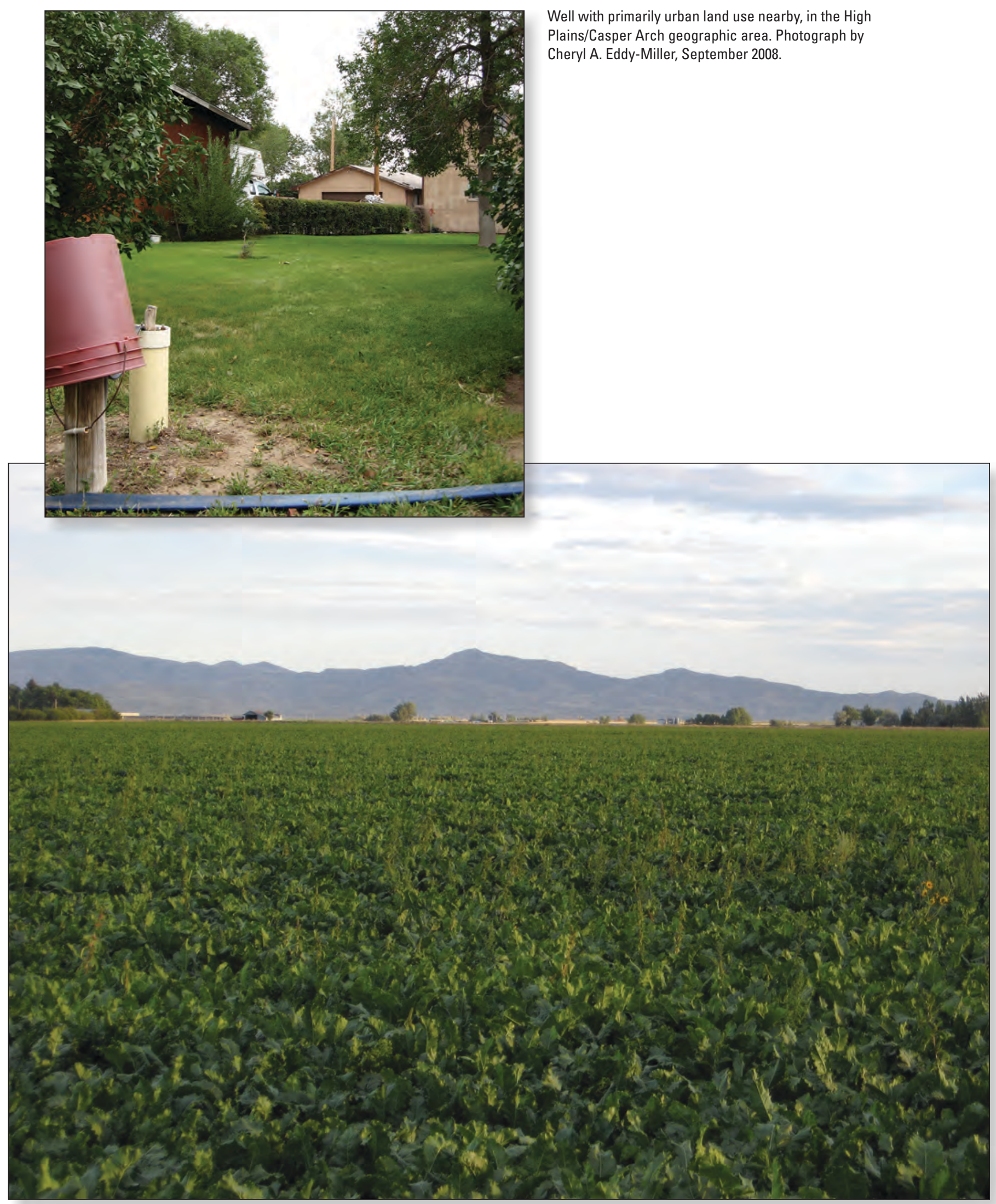

Sugar beet field in the Bighorn Basin geographic area. The pesticide detection frequency within the Bighorn Basin geographic area had the largest change from the 1995-2006 baseline study to the 2008-10 resampling study, changing from 56 percent to 17 percent. Photograph by Cheryl A. Eddy-Miller, September 2009. 

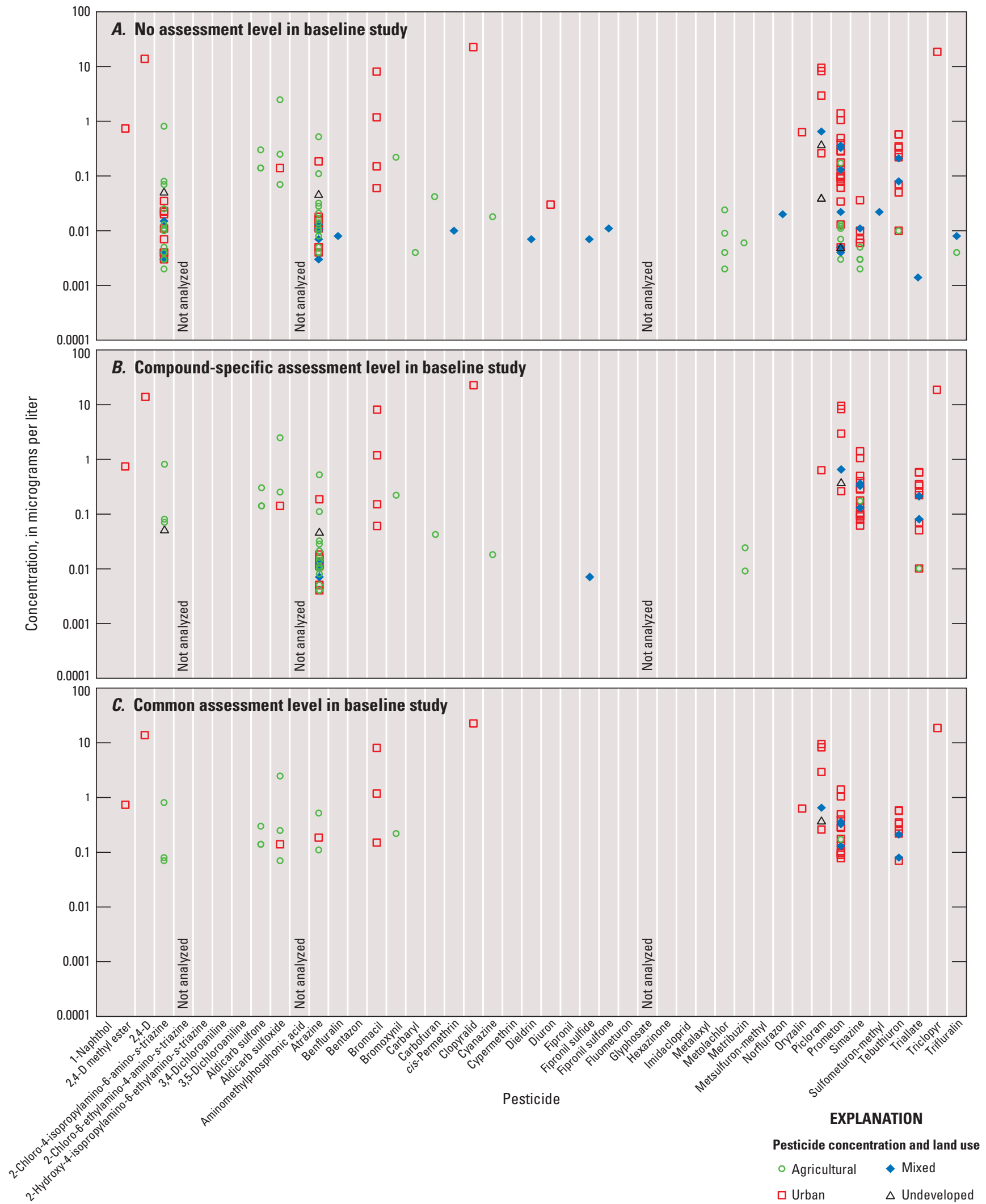

Figure 8. Pesticide detections and concentrations in groundwater samples collected from 52 wells during $1995-2006$ baseline study (fall samples only; Bartos and others, 2009) and 2008-10 resampling study, Wyoming. 


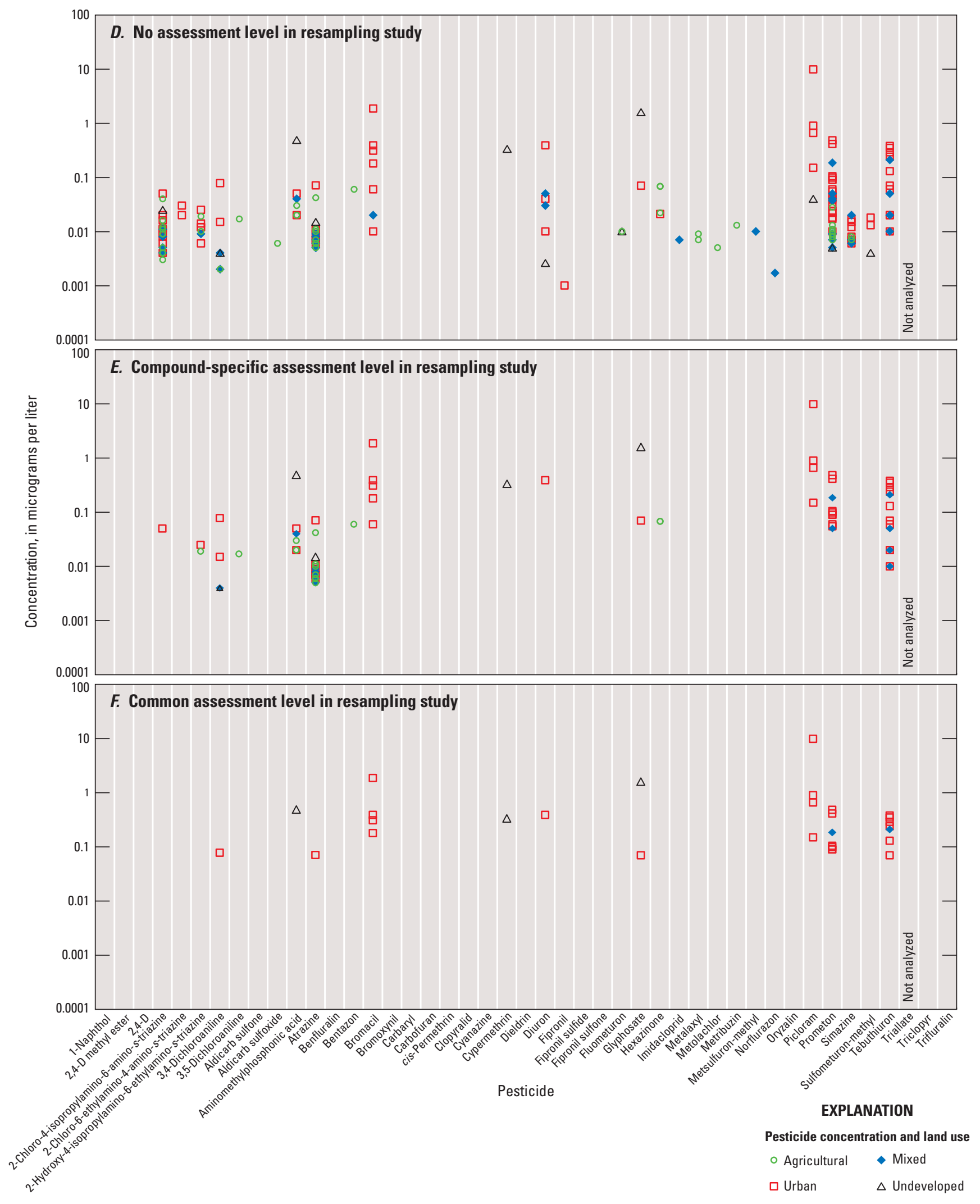

Figure 8. Pesticide detections and concentrations in groundwater samples collected from 52 wells during 1995-2006 baseline study (fall samples only; Bartos and others, 2009) and 2008-10 resampling study, Wyoming.-Continued 

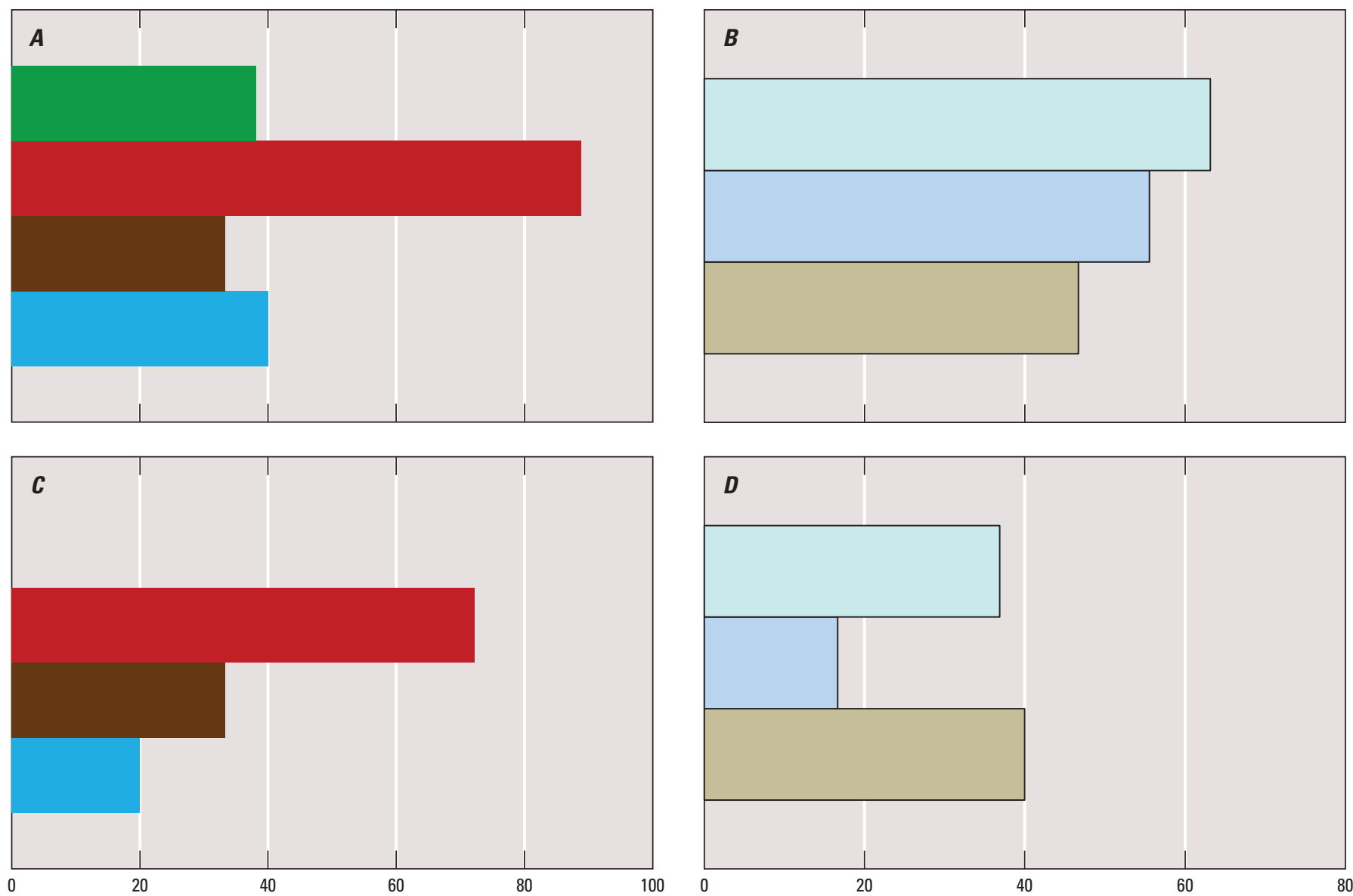

Percentage of wells within a land-use category with at least one pesticide detected at a concentration greater than the common assessment level (CAL)

Percentage of wells within a geographic area with at least one pesticide detected at a concentration greater than the common assessment level (CAL)

\section{EXPLANATION}

Land use

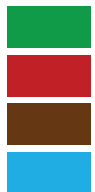

Agricultural

Urban

Undeveloped

Mixed

\section{EXPLANATION}

\section{Geographic area}

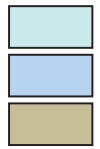

High Plains/Casper Arch

Bighorn Basin

Rest of Wyoming

Figure 9. Percentage of wells with at least one pesticide detected in groundwater samples collected from 52 wells relative to land use and geographic area during the 1995-2006 baseline study (fall samples only; Bartos and others, 2009) and the 2008-10 resampling study, Wyoming. A, Detections relative to land use, 1995-2006 baseline study. B, Detections relative to geographic area, 1995-2006 baseline study. $C$, Detections relative to land use, 2008-10 resampling study. D, Detections relative to geographic area, 2008-10 resampling study. 


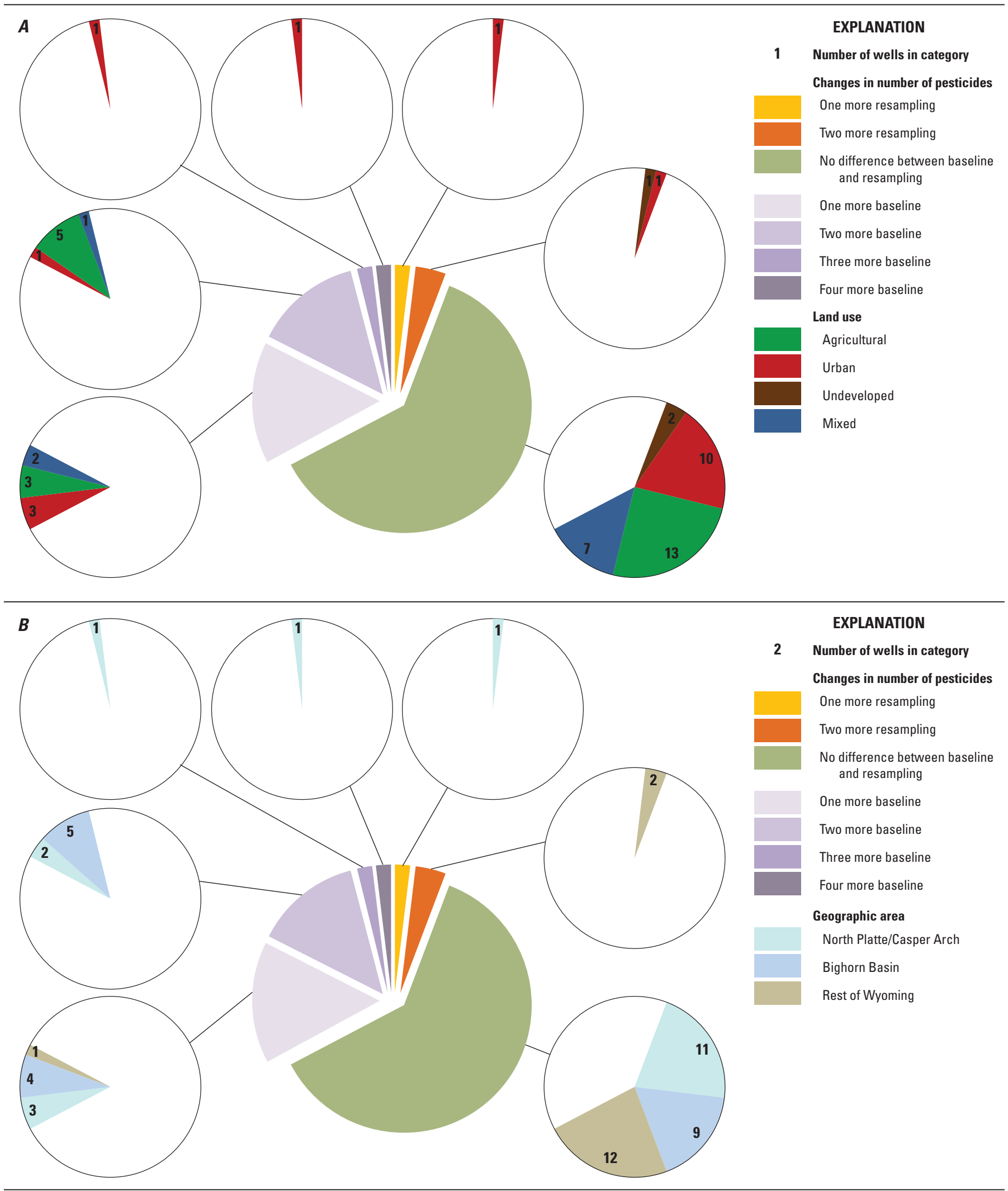

Figure 10. Difference in number of pesticides detected in the same well between 1995-2006 baseline (fall samples only; Bartos and others, 2009) and 2008-10 resampling studies. $A$, Land use near wells. B, Geographic area of well in each category. Pesticide detection is based on the common assessment level (CAL). 


\section{Summary}

The Ground-water and Pesticide Strategy Committee (GPSC) consisting of members of local, State, and Federal government, as well as industry and interest groups, prepared the State of Wyoming Generic Management Plan for Pesticides in Ground Water. A key component of this plan was to conduct baseline groundwater sampling to characterize pesticide occurrence in Wyoming's groundwater.

The U.S. Geological Survey (USGS), in cooperation with the Wyoming Department of Agriculture (WDA), acting on behalf of the GPSC, later with assistance from the Wyoming Department of Environmental Quality (WDEQ), began statewide implementation of this baseline groundwater sampling in 1995. During 1995-2006, samples were collected from 296 wells in Wyoming. Results of this study were previously summarized to describe the occurrence of pesticides in the State of Wyoming, and were statistically evaluated to determine occurrence of pesticides in relation to selected natural and anthropogenic characteristics. During 2008-10, the USGS, in cooperation with the WDA, resampled a subset of 52 wells that were sampled during the 1995-2006 baseline study in order to compare detected compounds and respective concentrations between the two sampling periods, and to evaluate the occurrence of new pesticides. The 52 wells were distributed similarly to sites used in the 1995-2006 baseline study with respect to geographic area and land use within the geographic area of interest. In order to help evaluate changes in pesticide occurrence, wells with previous detections of pesticides were given priority for resampling.

Because of the use of different types of "reporting levels" and variability in reporting-level values during the 1995-2006 baseline study and the 2008-10 resampling study, analytical results received from the laboratory were recensored. The recensoring techniques and values used for the resampling study were the same as used during the baseline study. In addition to using no assessment level, two levels of recensoring were used to compare pesticides - a compound-specific assessment level (CSAL) that differed by compound and a common assessment level (CAL) of 0.07 microgram per liter.

Twenty eight different pesticides were detected using no assessment level in samples from the 52 wells during the 2008-10 resampling study. Concentrations of detected pesticides generally were small (less than 1 microgram per liter), although three pesticides - bromacil, glyphosate, and picloram-were measured at concentrations greater than 1 microgram per liter one time each in groundwater from three different wells.

Pesticide concentrations were compared with several U.S. Environmental Protection Agency (USEPA) drinkingwater standards or health advisories for finished (treated) water established under the Safe Drinking Water Act. All detected pesticide concentrations were smaller than USEPA drinking-water standards or health advisories where applicable (many pesticides did not have standards or advisories). Most pesticide concentrations were at least an order of magnitude smaller than USEPA standards or health advisories.

The overall detection frequency of pesticides in groundwater from the 52 wells was examined by calculating the percentage of wells with at least one pesticide detected at concentrations greater than the CAL. One or more pesticides were detected at concentrations greater than the CAL in water from 16 of 52 wells sampled (about 31 percent) during the resampling study.

Detected pesticides were classified into one of six actions: herbicides, herbicide degradates, insecticides, insecticide degradate, fungicides, or fungicide degradates. At least 95 percent of detected pesticides were classified as herbicides or herbicide degradates.

The purpose of the 2008-10 resampling study was to determine if the specific pesticides detected in groundwater changed over time, and if the concentrations of previously detected pesticides changed as well. The data from the 52 wells sampled during the 2008-10 resampling study were compared to fall samples collected during the 1995-2006 baseline study.

The number of different pesticides detected in samples from the 52 wells was similar between the baseline study (30 different pesticides) and the resampling study (28 different pesticides). Thirteen pesticides were common to both sampling periods. During the baseline study, 16 additional pesticides were detected that were not detected during the resampling study. Fifteen pesticides were detected during the resampling study that had not been detected in the fall samples from the 52 wells sampled in the baseline study; however, three of these pesticides were not on the analytical schedules used during the baseline study. Most pesticides detected in one of the studies, but not the other, were only detected in one or two samples.

The change in the number of pesticides detected (without regard to which pesticide was detected) in groundwater samples from each of the 52 wells was calculated, and most of the wells (32) had no changes in the number of pesticides detected. Of those wells that did have a difference between the two studies, 17 wells had more pesticide detections in groundwater during the baseline study, whereas only 3 wells had more detections during the resampling study.

The change in concentration over time in groundwater samples from the 52 wells was determined by evaluating pesticides with at least 50 analyses during both sampling studies, and with at least one concentration greater than the CSAL. Most pesticides with detections had little change in concentration between the baseline study and resampling study. Seven pesticides had a greater concentration detected in groundwater from the same well during the baseline study than during the resampling study. Concentrations of atrazine, 
which was detected in 33 wells, and the atrazine degradate 2-chloro-4-isopropylamino-6-amino-s-triazine were greater in the baseline study sample than in the resampling study sample about 80 percent of the time. Concentrations of prometon, detected in 17 wells, were greater in the baseline study sample than in the resampling study sample 100 percent of the time.

The overall detection frequency of pesticides in groundwater from the 52 wells was examined by calculating the percentage of wells with at least one pesticide detected at concentrations greater than the CAL with respect to land use near the well and geographic area. The detection frequency of at least one pesticide in groundwater from a well decreased or remained unchanged, regardless of land use or geographic area, between the 1995-2006 baseline and 2008-10 resampling study. The detection frequency within the agricultural land-use category had the largest change between the 1995-2006 baseline and 2008-10 resampling studies, decreasing from 38 percent to zero. The detection frequency within the Bighorn Basin geographic area had the largest change between the 1995-2006 baseline and 2008-10 resampling studies, decreasing from 56 percent to 17 percent.

The change in the number of pesticides detected (without regard to which pesticide was detected) between the 1995-2006 baseline study and the 2008-10 resampling study in groundwater samples from each of the 52 wells with respect to land use and geographic area was calculated. All wells with land use classified as agricultural had the same or a smaller number of pesticides detected in the resampling study compared to the baseline study. All wells in the Bighorn Basin geographic area also had the same or a smaller number of pesticides detected in the resampling study compared to the baseline study.

\section{References Cited}

Bartos, T.T., Eddy-Miller, C.A., and Hallberg, L.L., 2009, Occurrence of pesticides in ground water of Wyoming, 1995-2006: U.S. Geological Survey Scientific Investigations Report 2009-5024, 72 p. (Also available at http:// pubs.usgs.gov/sir/2009/5024/.)

Childress, C.J. Oblinger, Foreman, W.T., Connor, B.F., and Maloney, T.J., 1999, New reporting procedures based on long-term method detection levels and some considerations for interpretations of water-quality data provided by the U.S. Geological Survey National Water Quality Laboratory: U.S. Geological Survey Open-File Report 99-193, 20 p.

Eddy-Miller, C.A., Bartos, T.T., Hallberg, L.L., 2009, Pesticides in ground water of Wyoming, 1995-2006: U.S. Geological Survey Fact Sheet 2009-3006, 4 p. (Also available at http://pubs.usgs.gov/fs/2009/3006/.)
Furlong, E.T., Anderson, B.D., Werner, S.L., Soliven, P.P., Coffey, L.J., and Burkhardt, M.R., 2001, Methods of analysis by the U.S. Geological Survey National Water Quality Laboratory-Determination of pesticides in water by graphitized carbon-based solid-phase extraction and high-performance liquid chromatography/mass spectrometry: U.S. Geological Survey Water-Resources Investigations Report 01-4134, 73 p.

Koterba, M.T., 1998, Ground-water data-collection protocols and procedures for the National Water-Quality Assessment Program - Collection, documentation, and compilation of required site, well, subsurface, and landscape data for wells: U.S. Geological Survey Water-Resources Investigations Report 98-4107, 91 p.

Koterba, M.T., Wilde, F.D., and Lapham, W.W., 1995, Ground-water data-collection protocols and procedures for the National Water-Quality Assessment Program - Collection and documentation of water-quality samples and related data: U.S. Geological Survey Open-File Report 95-399, $113 \mathrm{p}$.

Madsen, J.E., Sandstrom, M.W., and Zaugg, S.D., 2003, Methods of analysis by the U.S. Geological Survey National Water Quality Laboratory_A method supplement for the determination of fipronil and degradates in water by gas chromatography/mass spectrometry: U.S. Geological Survey Open-File Report 02-462, 11 p.

Meyer, M.T., Loftin, K.A., Lee, E.A., Hinshaw, G.H., Dietze, J.E., Scribner, E.A., 2009, Determination of glyphosate, its degradation product aminomethylphosphonic acid, and glufosinate, in water by isotope dilution and online solid-phase extraction and liquid chromatography/tandem mass spectrometry: U.S. Geological Survey Techniques and Methods, book 5, chap. A10, 32 p.

Nakagaki, Naomi, and Wolock, D.M., 2005, Estimation of agricultural pesticide use in drainage basins using land cover maps and county pesticide data: U.S. Geological Survey Open-File Report 2005-1188, 46 p.

Sandstrom, M.W., Stroppel, M.E., Foreman, W.T., and Schroeder, M.P., 2001, Methods of analysis by the U.S. Geological Survey National Water Quality Laboratory-Determination of moderate-use pesticides and selected degradates in water by $\mathrm{C}-18$ solid-phase extraction and gas chromatography/mass spectrometry: U.S. Geological Survey WaterResources Investigations Report 01-4098, 70 p.

Timme, P.J., 1995, National Water Quality Laboratory 1995 services catalog: U.S. Geological Survey Open-File Report 95-352, $92 \mathrm{p}$. 
U.S. Environmental Protection Agency, 2012, 2012 Edition of the drinking water standards and health advisories: Washington, D.C., U.S. Environmental Protection Agency, Office of Water, EPA 822-S-12-001, spring 2012, 20 p., accessed August 24, 2012, at http://www.epa.gov/waterscience/ criteria/drinking/dwstandards.pdf.

U.S. Geological Survey, variously dated, National field manual for the collection of water-quality data: U.S. Geological Survey Techniques of Water-Resources Investigations, book 9, chaps. A1-A9, 2 v., variously paged, accessed August 26, 2012, at http://pubs.water.usgs.gov/twri9A.

U.S. Geological Survey, 2012, National Water Information System, accessed August 26, 2012, at http://nwis.waterdata. usgs.gov/wy/nwis/qwdata.

Wyoming Ground-water and Pesticides Strategy Committee, 1999, Wyoming generic State management plan for pesticides and ground water: Cheyenne, Wyo., prepared for Wyoming Department of Agriculture, $103 \mathrm{p}$.

Zaugg, S.D., Sandstrom, M.W., Smith, S.G., and Fehlberg, K.M., 1995, Methods of analysis by the U.S. Geological Survey National Water Quality Laboratory-Determination of pesticides in water by $\mathrm{C}-18$ solid-phase extraction and capillary-column gas chromatography/mass spectrometry with selected-ion monitoring: U.S. Geological Survey Open-File Report 95-181, 49 p. 
Appendixes 
Appendix 1. Blank data for sample collected at site 410817104470201, November 2009.

$[\mu \mathrm{g} / \mathrm{L}$, micrograms per liter; $<$, less than $]$

\begin{tabular}{lc}
\hline \multicolumn{1}{c}{ Pesticide } & $\begin{array}{c}\text { Concentration } \\
(\boldsymbol{\mu} \mathbf{g} / \mathbf{L})\end{array}$ \\
\hline Acrylonitrile & $<0.80$ \\
Bromacil & $<0.36$ \\
Bromomethane & $<0.2$ \\
Carbaryl & $<0.38$ \\
Carbazole & $<0.030$ \\
Carbon disulfide & $<0.04$ \\
Chlorpyrifos & $<0.16$ \\
cis-1,3-Dichloropropene & $<0.10$ \\
DEET & $<0.06$ \\
Diazinon & $<0.16$ \\
Iodomethane & $<0.26$ \\
Metalaxyl & $<0.12$ \\
Metolachlor & $<0.080$ \\
Prometon & $<0.12$ \\
trans-1,3-Dichloropropene & $<0.14$ \\
\hline
\end{tabular}

Appendix 2. Replicate sample data for detected pesticides from samples collected at sites 4108108104223501 (September 2009 ) and 444603108383401 (September 2010). All other pesticides analyzed for had no detection in either the environmental or replicate sample.

[RPD, relative percent difference; $\mu \mathrm{g} / \mathrm{L}$, micrograms per liter; E, estimated value; $<$, less than; $\mathrm{NC}$, not calculated]

\begin{tabular}{|c|c|c|c|c|c|c|}
\hline \multirow[b]{2}{*}{ Pesticide } & \multicolumn{3}{|c|}{ Samples for site 410108104223501 (Lr4) } & \multicolumn{3}{|c|}{ Samples for site 444603108383401 (Pk1) } \\
\hline & $\begin{array}{c}\text { Environmental } \\
(\mu \mathrm{g} / \mathrm{L})\end{array}$ & $\begin{array}{c}\text { Replicate } \\
\text { ( } \mu \mathrm{g} / \mathrm{L})\end{array}$ & $\begin{array}{c}\text { RPD } \\
\text { percent }\end{array}$ & $\begin{array}{c}\text { Environmental } \\
(\mu \mathrm{g} / \mathrm{L})\end{array}$ & $\begin{array}{c}\text { Replicate } \\
\text { ( } \mu \mathrm{g} / \mathrm{L})\end{array}$ & $\begin{array}{c}\text { RPD } \\
\text { percent }\end{array}$ \\
\hline 3,4-Dichloroaniline & E0.004 & E0.0035 & 13 & $<0.004$ & $<0.004$ & $\mathrm{NC}$ \\
\hline Atrazine & 0.015 & 0.014 & 7 & $<0.007$ & $<0.007$ & $\mathrm{NC}$ \\
\hline Fluometuron & E0.01 & $<0.04$ & $\mathrm{NC}$ & E0.01 & E0.01 & 0 \\
\hline Picloram & E0.04 & E0.04 & 0 & $<0.12$ & $<0.12$ & $\mathrm{NC}$ \\
\hline Sulfometuron-methyl & E0.004 & E0.003 & 29 & $<0.060$ & $<0.060$ & $\mathrm{NC}$ \\
\hline
\end{tabular}


Appendix 3. Spike recovery data from sample collected from site 41101910405130 (Lr7), September 2008.

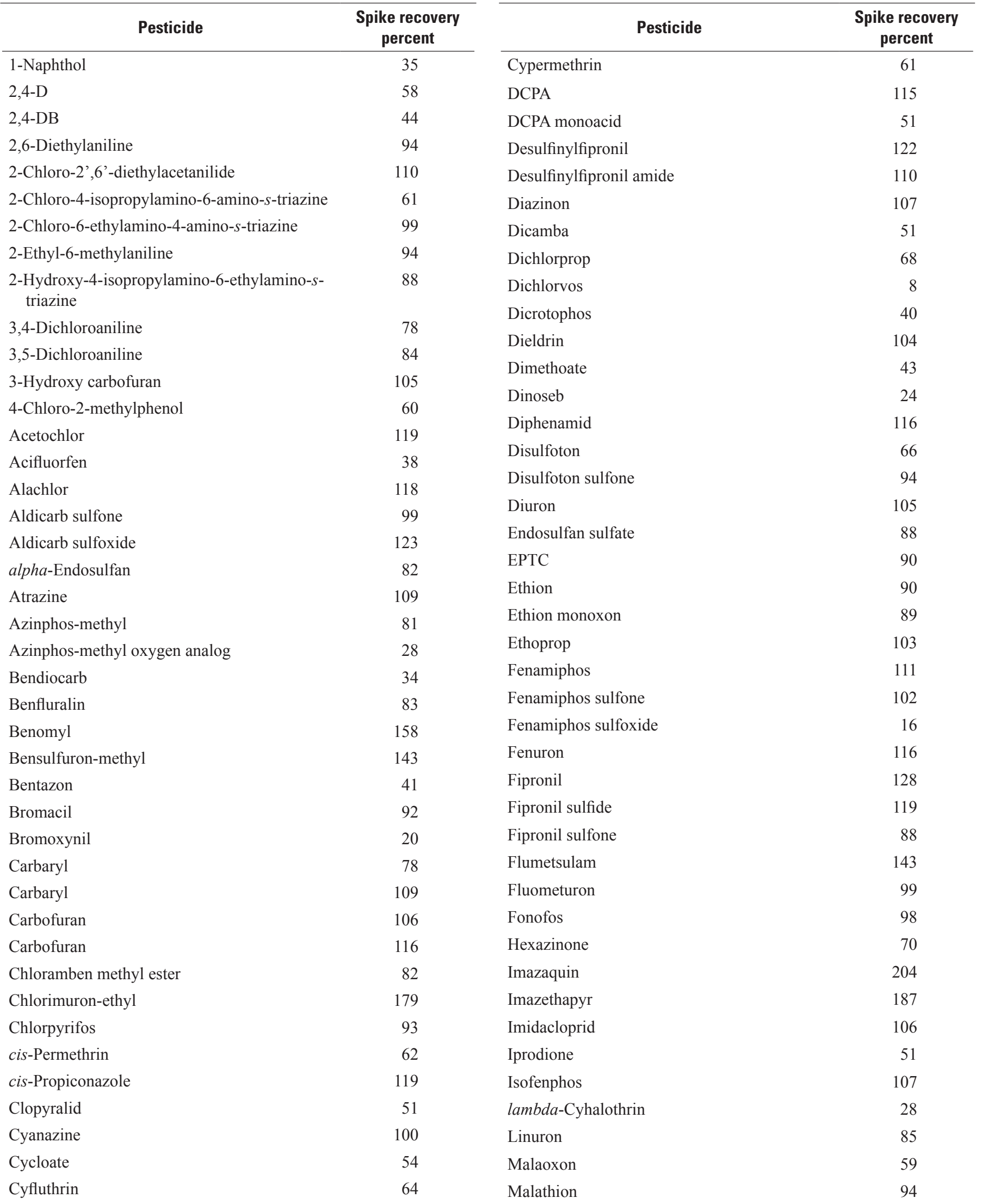


Appendix 3. Spike recovery data from sample collected from site 41101910405130 (Lr7), September 2008.—Continued

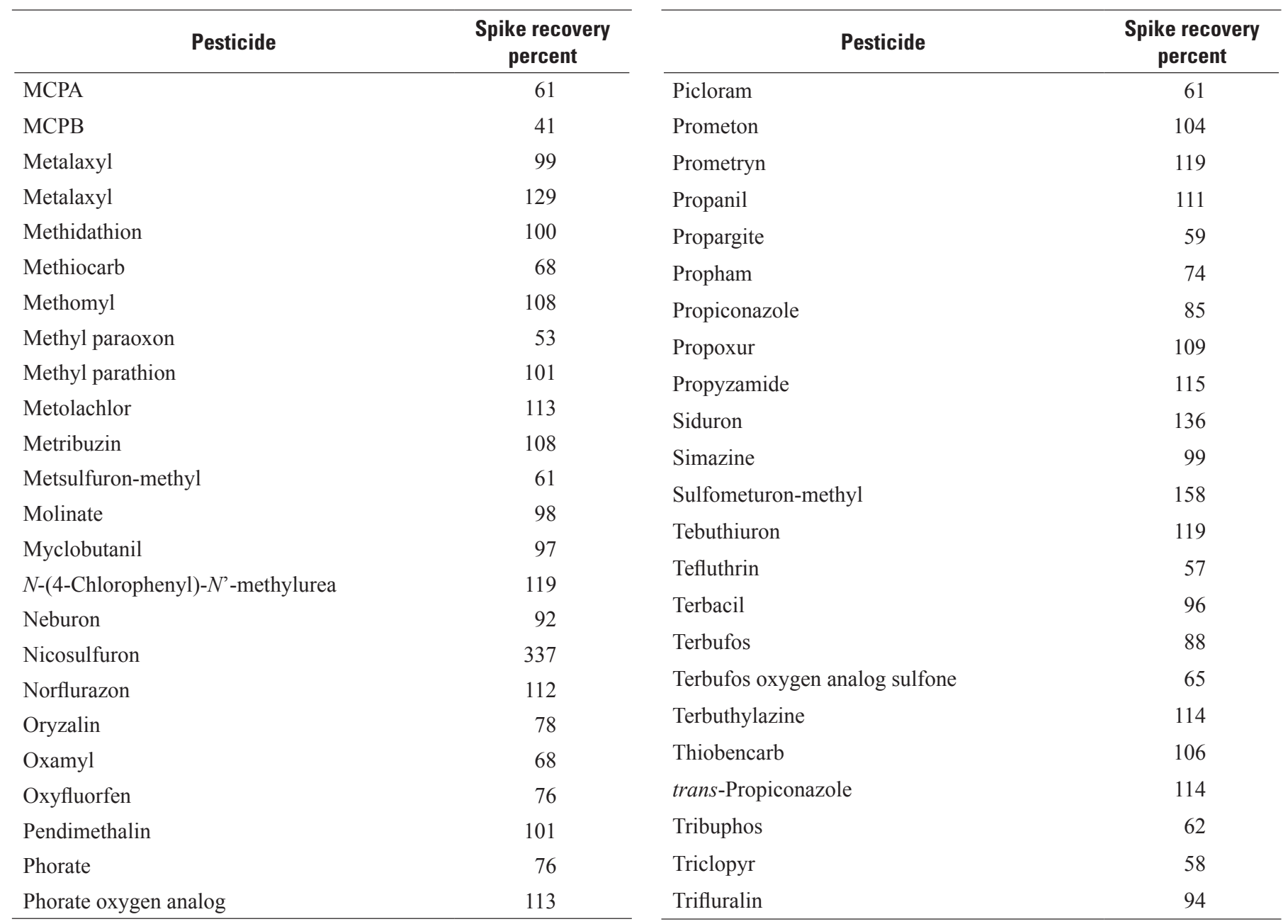


Appendix 4. Name, number, and ancillary information for 52 wells sampled during 1995-2006 baseline study' and 2008-10 resampling study, Wyoming.

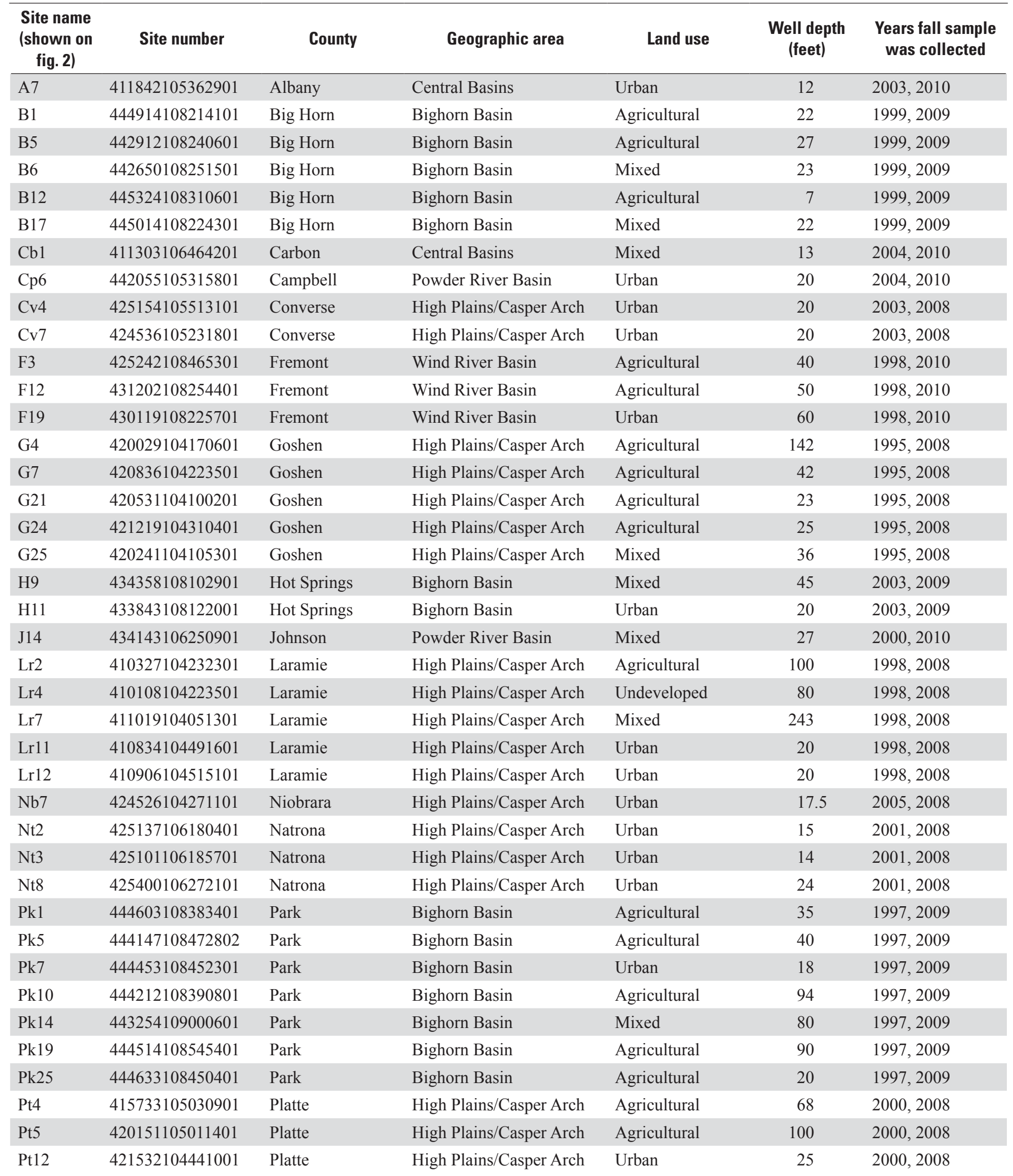


Appendix 4. Name, number, and ancillary information for 52 wells sampled during 1995-2006 baseline study ${ }^{1}$ and $2008-10$ resampling study, Wyoming.-Continued

\begin{tabular}{|c|c|c|c|c|c|c|}
\hline $\begin{array}{c}\text { Site name } \\
\text { (shown on } \\
\text { fig. 2) }\end{array}$ & Site number & County & Geographic area & Land use & $\begin{array}{l}\text { Well depth } \\
\text { (feet) }\end{array}$ & $\begin{array}{c}\text { Years fall sample } \\
\text { was collected }\end{array}$ \\
\hline $\mathrm{Sb} 3$ & 425633110142401 & Sublette & Green River Basin & Mixed & 90 & 2004,2010 \\
\hline $\operatorname{Sh} 3$ & 444338106161001 & Sheridan & Powder River Basin & Undeveloped & 30 & 1999,2010 \\
\hline Sh5 & 444645106574001 & Sheridan & Powder River Basin & Urban & 24 & 1999,2010 \\
\hline Sh15 & 445014106490701 & Sheridan & Powder River Basin & Agricultural & 50 & 1999,2010 \\
\hline Sw9 & 420635109270101 & Sweetwater & Green River Basin & Undeveloped & 80 & 2001,2010 \\
\hline $\mathrm{U} 2$ & 411643110583401 & Uinta & Overthrust Belt & Mixed & 35 & 2002,2010 \\
\hline U10 & 411937110172801 & Uinta & Green River Basin & Urban & 13 & 2002,2010 \\
\hline Wk3 & 440031107573701 & Washakie & Bighorn Basin & Urban & 30 & 1997,2009 \\
\hline
\end{tabular}

${ }^{1}$ Bartos and others (2009). 
Appendix 5. Summary of pesticides distributed within Wyoming, 2007.'

[NAL, no assessment level; H, Herbicide; F, Fungicide; I, Insecticide; R, Rodenticide; L, Larvicide; S, Soil fumigant; ST, Seed treatment; CP, Crop protectant; GR, Growth regulator; B, Bactericide, Algaecide; MCPA, (4-chloro-2-methylphenoxy)acetic acid; EPTC, S-ethyl dipropylthiocarbamate; DCPA, dimethyl tetrachloroterephthalate; MSMA, monosodium methyl arsenate; PCNB, pentachloronitrobenzene; --, not on analytical schedule]

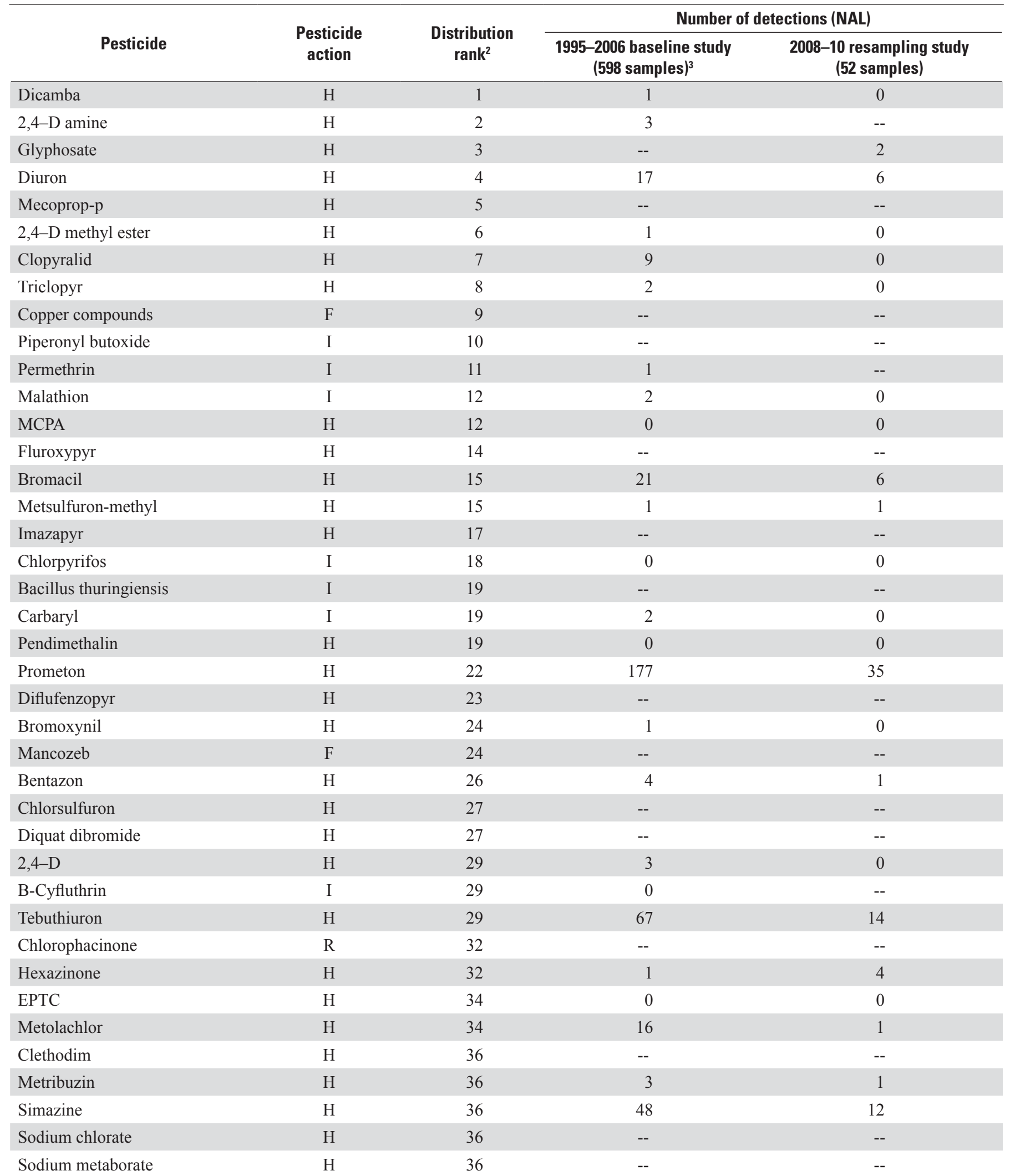


Appendix 5. Summary of pesticides distributed within Wyoming, 2007.'- Continued

[NAL, no assessment level; H, Herbicide; F, Fungicide; I, Insecticide; R, Rodenticide; L, Larvicide; S, Soil fumigant; ST, Seed treatment; CP, Crop protectant; GR, Growth regulator; B, Bactericide, Algaecide; MCPA, (4-chloro-2-methylphenoxy)acetic acid; EPTC, S-ethyl dipropylthiocarbamate; DCPA, dimethyl tetrachloroterephthalate; MSMA, monosodium methyl arsenate; PCNB, pentachloronitrobenzene; --, not on analytical schedule]

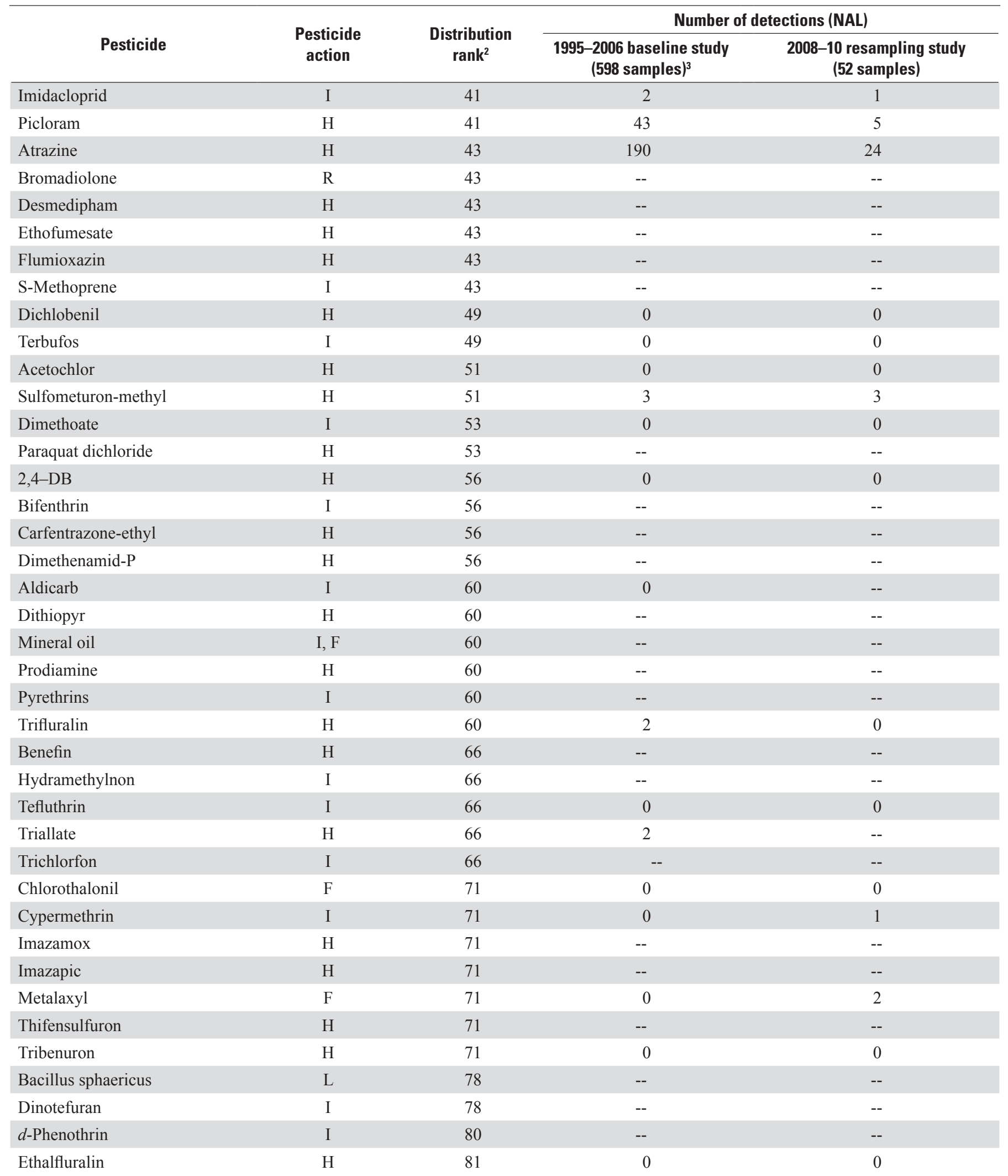


Appendix 5. Summary of pesticides distributed within Wyoming, 2007. ${ }^{1}$-Continued

[NAL, no assessment level; H, Herbicide; F, Fungicide; I, Insecticide; R, Rodenticide; L, Larvicide; S, Soil fumigant; ST, Seed treatment; CP, Crop protectant; GR, Growth regulator; B, Bactericide, Algaecide; MCPA, (4-chloro-2-methylphenoxy)acetic acid; EPTC, S-ethyl dipropylthiocarbamate; DCPA, dimethyl tetrachloroterephthalate; MSMA, monosodium methyl arsenate; PCNB, pentachloronitrobenzene; --, not on analytical schedule]

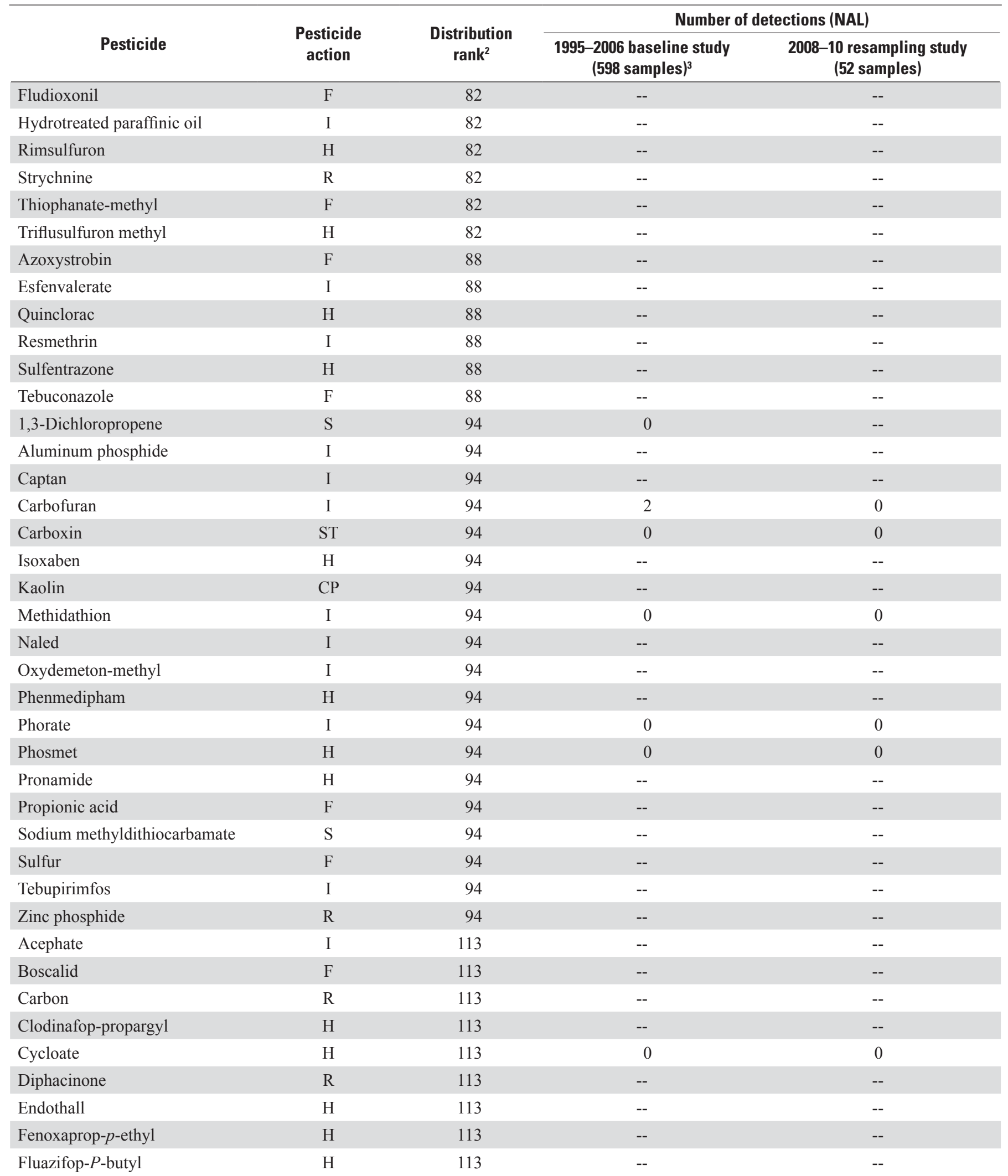


Appendix 5. Summary of pesticides distributed within Wyoming, 2007.'-Continued

[NAL, no assessment level; H, Herbicide; F, Fungicide; I, Insecticide; R, Rodenticide; L, Larvicide; S, Soil fumigant; ST, Seed treatment; CP, Crop protectant; GR, Growth regulator; B, Bactericide, Algaecide; MCPA, (4-chloro-2-methylphenoxy)acetic acid; EPTC, S-ethyl dipropylthiocarbamate; DCPA, dimethyl tetrachloroterephthalate; MSMA, monosodium methyl arsenate; PCNB, pentachloronitrobenzene; --, not on analytical schedule]

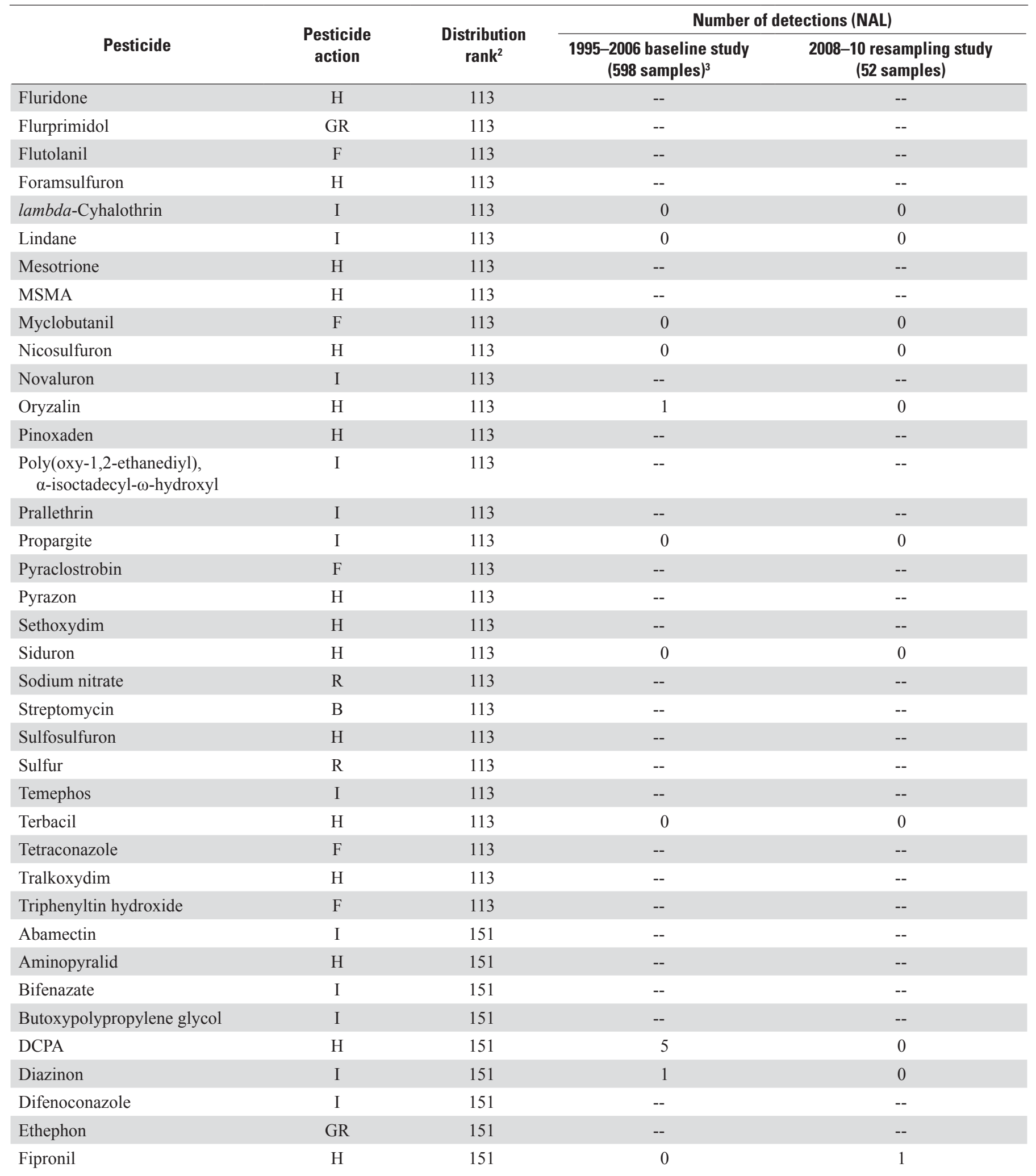


Appendix 5. Summary of pesticides distributed within Wyoming, 2007.'-Continued

[NAL, no assessment level; H, Herbicide; F, Fungicide; I, Insecticide; R, Rodenticide; L, Larvicide; S, Soil fumigant; ST, Seed treatment; CP, Crop protectant; GR, Growth regulator; B, Bactericide, Algaecide; MCPA, (4-chloro-2-methylphenoxy)acetic acid; EPTC, S-ethyl dipropylthiocarbamate; DCPA, dimethyl tetrachloroterephthalate; MSMA, monosodium methyl arsenate; PCNB, pentachloronitrobenzene; --, not on analytical schedule]

\begin{tabular}{|c|c|c|c|c|}
\hline \multirow[b]{2}{*}{ Pesticide } & \multirow{2}{*}{$\begin{array}{l}\text { Pesticide } \\
\text { action }\end{array}$} & \multirow{2}{*}{$\begin{array}{l}\text { Distribution } \\
\text { rank }^{2}\end{array}$} & \multicolumn{2}{|c|}{ Number of detections (NAL) } \\
\hline & & & $\begin{array}{c}\text { 1995-2006 baseline study } \\
\text { (598 samples) }^{3}\end{array}$ & $\begin{array}{c}\text { 2008-10 resampling study } \\
\text { (52 samples) }\end{array}$ \\
\hline Iprodione & $\mathrm{F}$ & 151 & 0 & 0 \\
\hline Oxyfluorfen & $\mathrm{H}$ & 151 & 1 & 0 \\
\hline Propiconazole & $\mathrm{F}$ & 151 & 0 & 0 \\
\hline Pyraflufen ethyl & $\mathrm{H}$ & 151 & -- & -- \\
\hline Quizalofop- $P$-ethyl & $\mathrm{H}$ & 151 & -- & -- \\
\hline Spiromesifen & I & 151 & -- & -- \\
\hline
\end{tabular}

${ }^{1}$ Data from Wyoming Department of Agriculture (Hank Uhden, Wyoming Department of Agriculture, written commun., 2010). Summary includes pesticides that were distributed in Wyoming in 2007. This does not necessarily mean the pesticide was sold or used during 2007.

${ }^{2} \mathrm{~A}$ ranking of 1 represents the active ingredient distributed most frequently. Progressively higher ranking numbers represent progressively lower distribution.

${ }^{3}$ Bartos and others (2009). 
Publishing support provided by the:

Denver, Pembroke, and Rolla Publishing Service Centers

For more information concerning this publication, contact: Director, Wyoming Water Science Center

U.S. Geological Survey

521 Progress Circle, Suite 6

Cheyenne, Wyoming 82007

(307) 778-2931

Or visit the Wyoming Water Science Center Web site at: http://wy.water.usgs.gov/ 



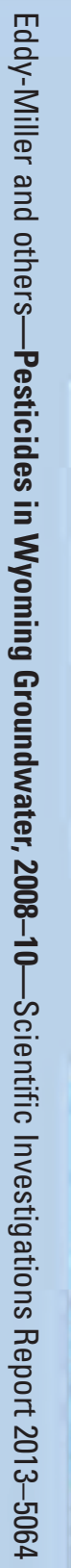

